Historic, archived document

Do not assume content reflects current scientific knowledge, policies, or practices. 

Pichard

(

Diener

INCORPORATED

Compoony

1

KENTFIELD, MARIN @OUNTY, CALIFORNIA

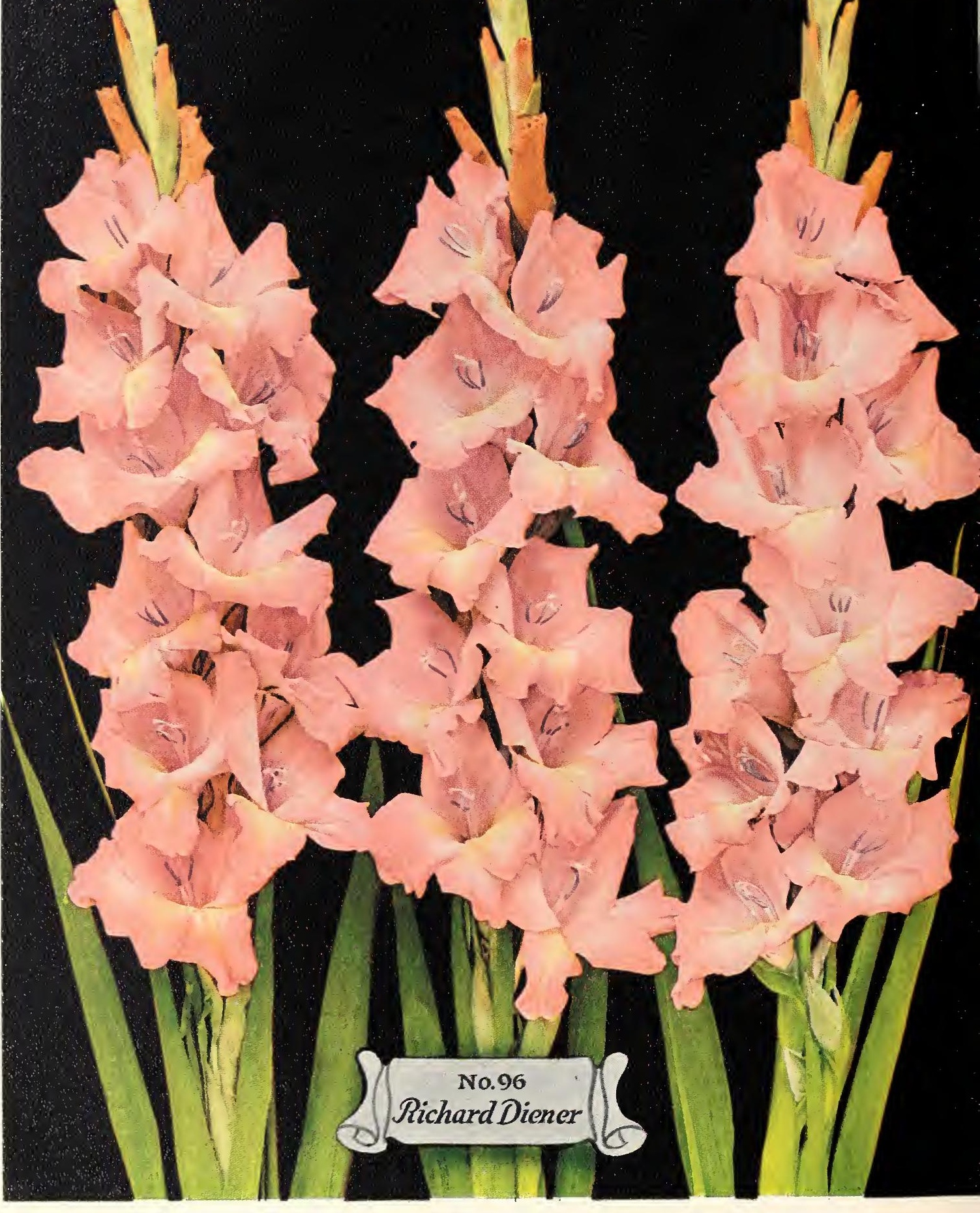


RICHARD DIENER CO., INC., KENTFIELD, MARIN COUNTY, CALIFORNIA

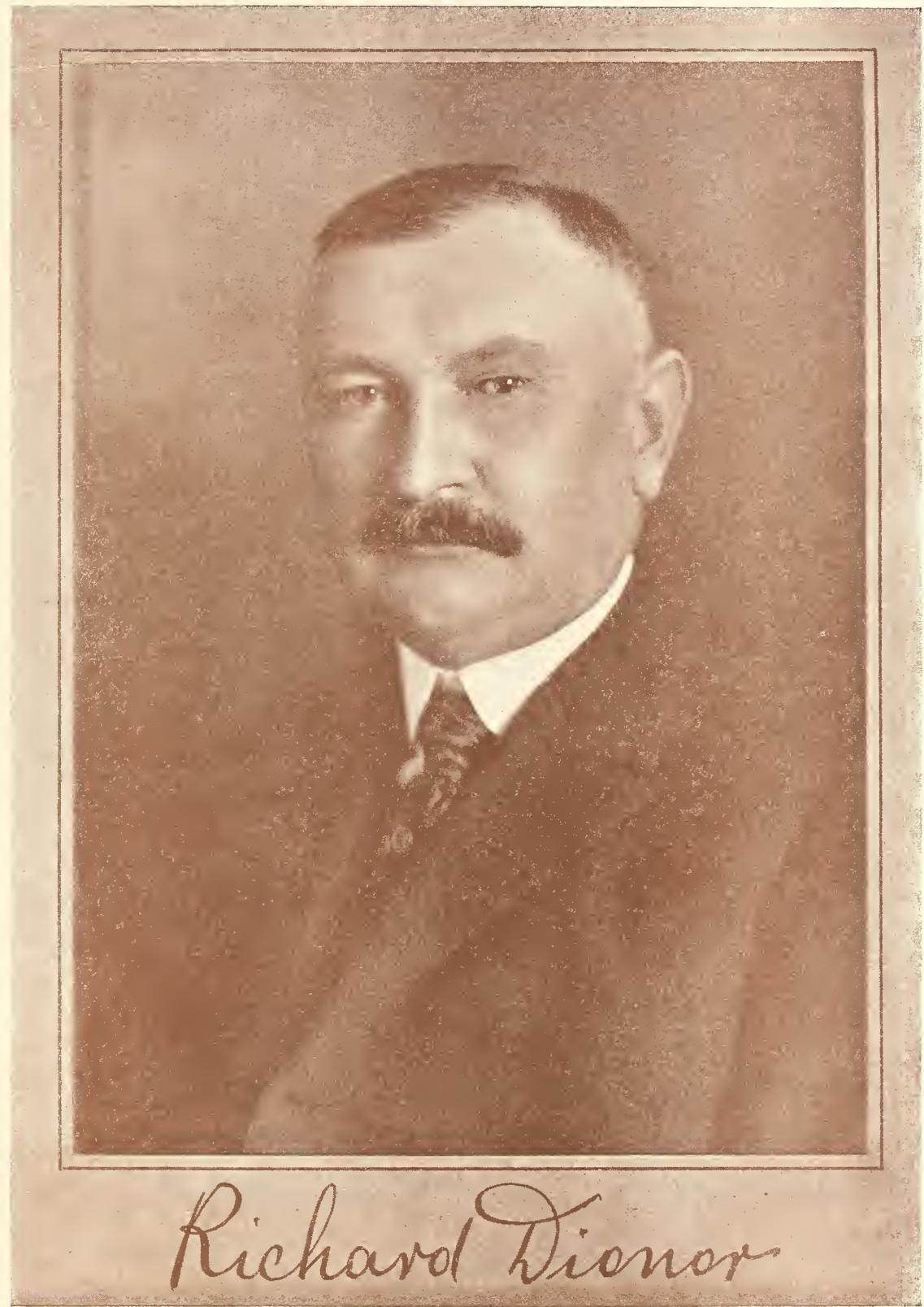


RICHARD DIENER CO., INC., KENTFIELD, MARIN COUNTY, CALIFORNIA
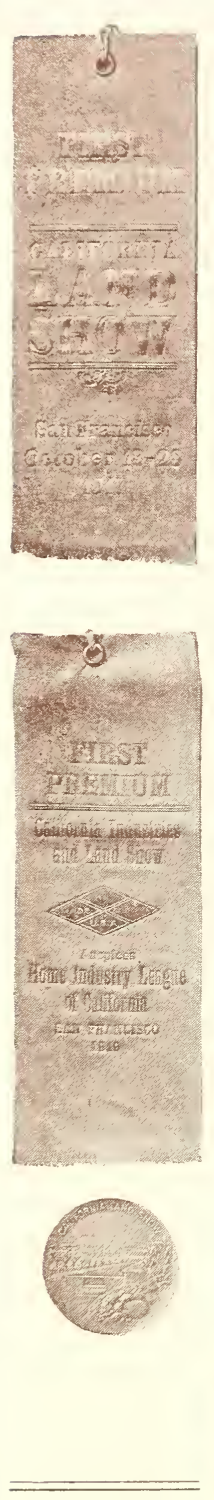

\section{AWARDS}

received

for the various

display of

vur products
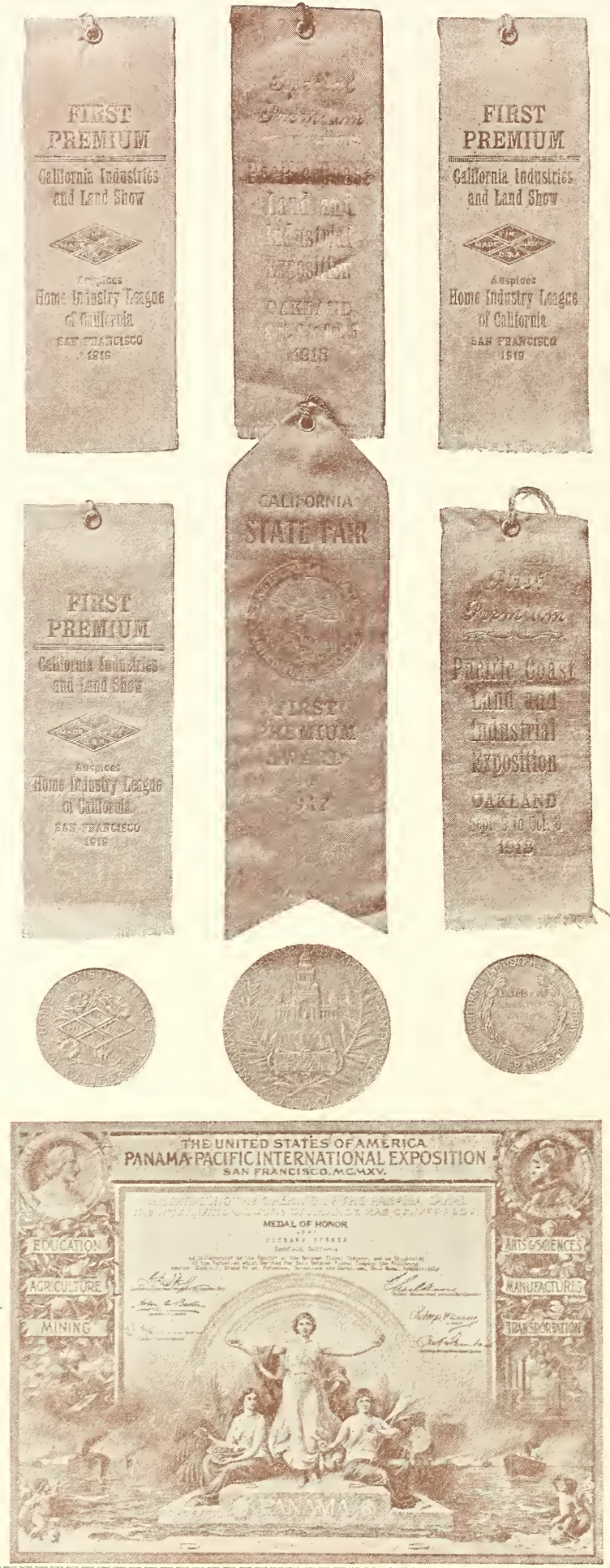
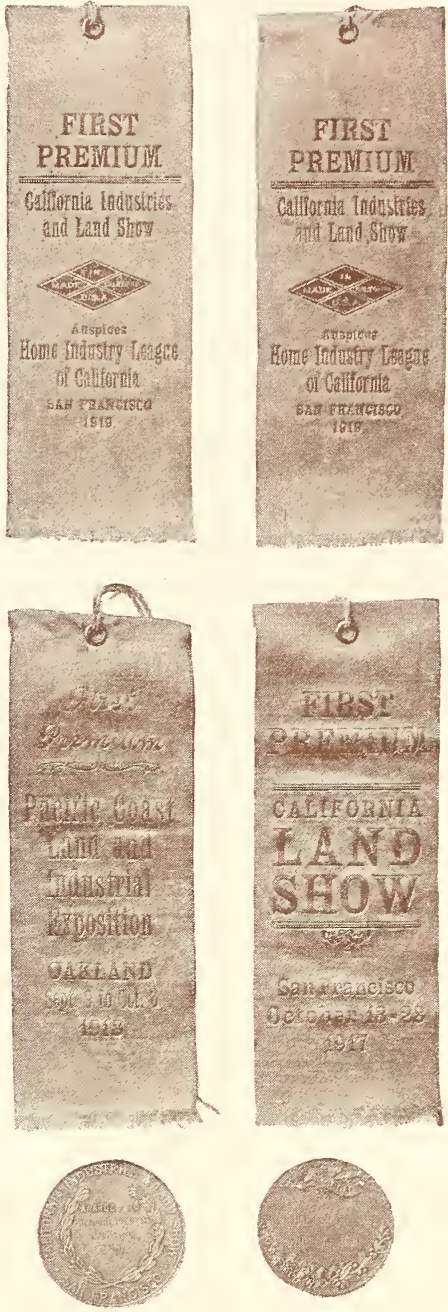

MEDAL OF HONOR

conferred upon

Richard Diener

at the

World's Fair

San Francisco

I915 


\title{
To Our Friends and Customers
}

\author{
We Present Our Annual Catalog for 1924
}

We also wish to thank you for the many kind letters and testimonials which we were unable to answer separately.

\section{RICHARD DIENER COMPANY.} catalog.

We carry no other than the Diener varieties of Gladioli listed in this

All the varieties listed are Mr. Diener's own productions, and in the description of colors, we have gone according to Ridgway's "COLOR STANDARDS AND NOMENCLATURE," giving only the fundamental colors as it is almost impossible to describe the shadings precisely.

Many people think that our superior climate here is responsible for our large sized flowers and magnificent colorings, but this is due to the variety and not to the climate.

As you will see by reading the testimonials, our varieties of Gladioli have been raised in all parts of the world just as successfully as they have been raised here, with the exception of a few of our varieties, which have not done so well the first year in a new locality, but the second year they have come up to catalog descriptions. Eastern varieties have acted in the same manner when brought to our California climate.

Our climate is the most suitable for producing perfectly ripened out bulbs, because after digging we leave them in the open air and full sun for at least four weeks. When so treated the bulbs will not sprout before being planted again, and they will be full of life and vigor. It is impossible in Northern and Eastern states to produce bulbs of such high quality, as the rains and early frosts there make such treatment impossible. Hence, the surprise of the people when they receive our bulbs.

At flower shows throughout this country, Canada and Australia during the past years, the Diener Hybrids continued to be the center of attraction. Such varieties as our Jack London, Anna Eberius, Mrs. H. E. Bothin, Diener's American Beauty, Richard Diener, Elf, Mrs. J. K. Armsby, D. J. Whitney, Thomas T. Kent, Geraldine Farrar, Mr. W. H. Phipps, William Kent and Dr. F. E. Bennett, and many others of our newer varieties proved themselves to be the finest of their kind and type. The new varieties which we offer are of such high perfection in color, size, growth and height of spikes that we can truthfully state that they are unexcelled anywhere. Wise are those that discard, old, wornout varieties and plant new and better ones. People are tired of old familiar kinds and are always willing to pay for new and better varieties. Too many kinds are put on the market each year that are worthless; but anything that we offer, you may rest assured that it is of the very best and highest quality.

During the flower season, which is from the end of June to September, we daily have visitors from all parts of the world, and we invite all lovers of flowers to visit our gardens. Kentfield is fourteen miles from San Francisco, on the Northwestern Pacific Railroad. It takes 55 minutes from the San Francisco Ferry Building to reach our place, which is a block from the Kentfield station.

Our place is closed on Sundays and holidays, but if notified by mail or phone a day or two ahead, the premises will be open. Telephone number, San Anselmo 114-J. 


\section{NOTICE TO FOREIGN CUSTOMERS}

Money orders from foreign countries should be made out to the post office at Kentfield, California, U. S. A.

\section{TERMS OF SALE}

While we exercise the utmost care to have our stock true to name, we give no warranty, express or implied, as to crop, and will not be responsible for results, other than to replace stock not true to name: However, we will not replace bulblets not true to name, except where the single bulb is $\$ 1.00$ or over.

Bulbs found to be not true to name should be retained by the customer and we replace number that are untrue, free of charge. No planting stock or bulblets will be included in this case.

Cash with orders or in advance of shipment. We positively accept no C. O. D. Orders. We will accept unused U. S. postage stamps up to the amount of two dollars $(\$ 2.00)$.

We do not ship plant or bulb orders for less than $\$ 1.00$.

Write your order plainly and distinctly by itself, and do not mix it with anything you wish to say. Be sure to write your NAME and ADDRESS plainly.

On orders amounting to more than $\$ 25.00$, we will give a discount made up of extra bulbs and seeds to the TRADE only.

We ship Gladioli bulbs from November 1st to May 1st. May 1st is our last day of planting.

We call a bulb anything from an inch or over in diameter.

\section{ORDER EARLY}

We strive to fill orders a day or two after they reach us. This is frequently made impossible during the great rush of business in February and March.

Do not wait until you need your seeds, order as early as possible.

We request our Northern and Eastern customers to send their orders in at an early date, but advise not to have the bulbs shipped them until after March 15 th, at which time all danger of heavy frosts will be over, and the bulbs will not be frozen in transit.

As we send orders out immediately, we will not send out acknowledgment of receipt of order, except in instances where goods are desired at a later date.

After goods reach post offices or express offices, our responsibility ceases.

We pack our shipments carefully, and there is hardly any danger of them being frozen in transit, but, if the package should arrive in zero weather, leave it in a cool, frost-protected place for at least two weeks before unpacking, so that if they should have been frozen, they will gradually thaw out. If they are unpacked in a frozen condition and brought in a warm place, they will be killed instantly, while when they are thawed out slowly in a cool place there will be no danger or injury to the bulbs, as they can stand quite a bit of frost.

\section{WE PAY ALL POSTAGE}

We recommend THE FLOWER GROWER to our friends and customers. Published monthly. All flower lovers should be regular subscribers. Subscription price: Three years, $\$ 3.50$; one year, $\$ 1.50$. Madison Cooper, Publisher, Calcium, New York.

\section{PLEASE READ VERY CAREFULLY BEFORE ORDERING}

1. We will be glad to make up special assortments of bulbs for our customers who may ask our aid in deciding what to plant. State your preference of color and the amount you desire, also varieties you have previously grown.

2. Send orders early to insure getting all the varieties desired, as our stocks are not of the same size and some of the varieties may be exhausted later in the season. Please use order blank enclosed in the Catalog for this purpose.

3. SUBSTITUTION : If any varieties are out of stock when your order is received, we reserve the right to substitute a similar variety.

4. In order that we may give our customers the opportunity of having as large a collection as possible of our varieties, we are offering bulbs, in large, one-half, one-quarter and bulblet sizes this season. However, we positively will not sell single bulbs in the small sizes except where the prices are given. Bulb. lets of nearly all varieties may be had in ONE DOZEN or ONE HUNDRED LOTS, and in singles only where designated. Please order in the amounts and quantities stated in the catalog. Prices on larger amounts of bulbs and bulblets, than shown in the Catalog, will be furnished at a much lower figure. Write us in regard to the same. 
RICHARD DIENER CO., INC., KENTFIELD, MARIN COUNTY, CALIFORNIA

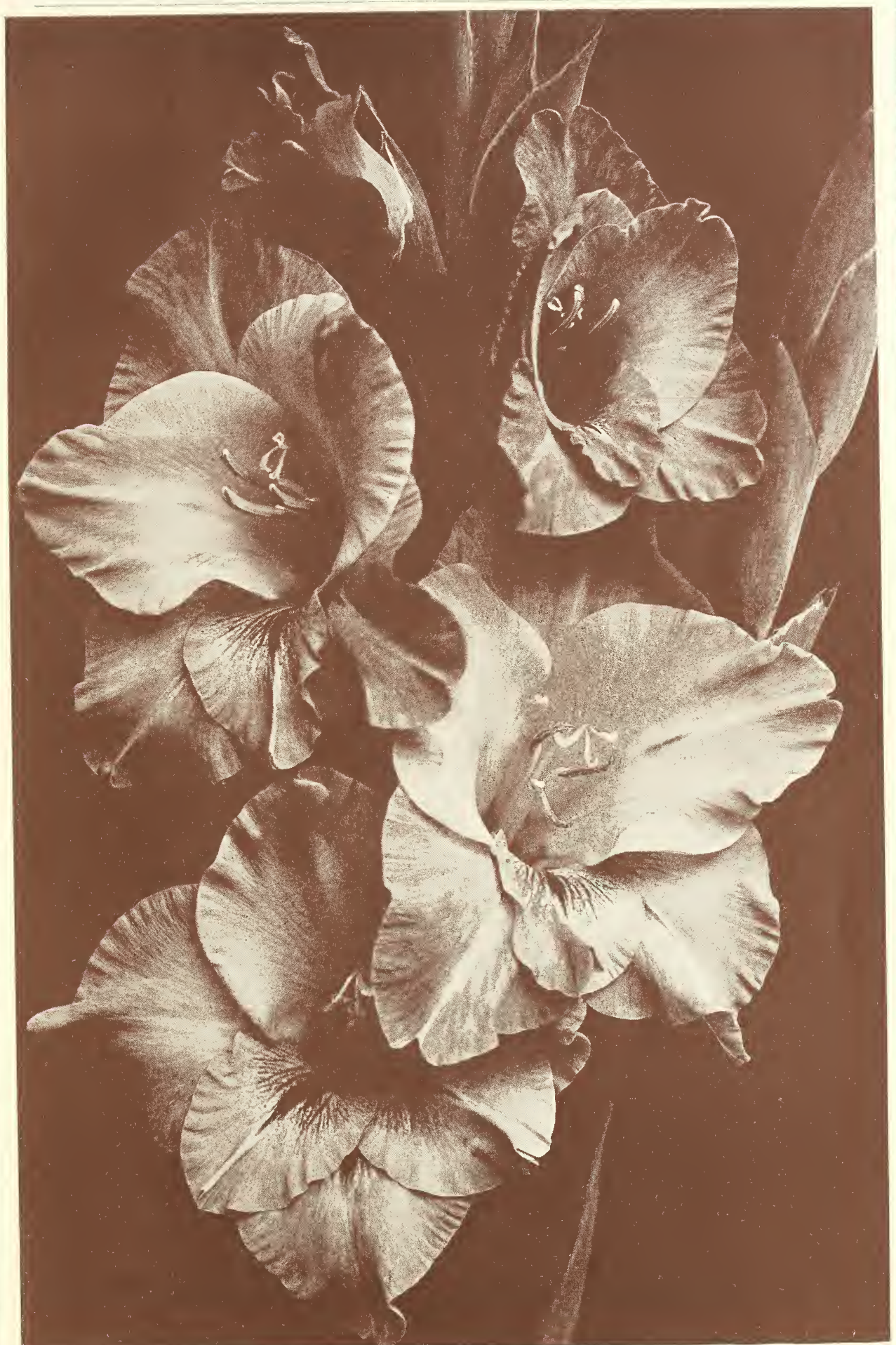




\section{Gladiolus Price List}

Bulbs and bulblets will be sold only in the amounts shown under each particular variety. In order to fill our orders promptly during the rush season, we are obliged to pack the bulbs in advance. For this reason, we positively cannot split the amounts shown in the catalog. If less than one hundred bulbs or bulblets are desired, they must be ordered in single or dozen lots.

We allow no discount on medium-sized bulbs or bulblets.

\section{No. 1- "MRS. WILLIAM KENT"}

Colors ranging from rose to shrimp pink, overlaid and striped with deeper rose pink. Throat straw color mottled with ruby. The color of this variety is exquisite and suitable for cut flowers. It is of very rapid growth and a heavy propagator. Flowers are large and spikes very tall.

$\begin{array}{lrccc} & \text { Bulbs } & \text { One-half Size } & \text { One-quarter Size } & \text { Bulblets } \\ \text { Per Bulb } & .15 & . .95 & .9 \% & \ldots \\ \text { Per Doz. } & 1.50 & .75 & .50 & .30 \\ \text { Per 100 } & 12.00 & 6.00 & 3.00 & 1.50\end{array}$

No. 4 "SARAH LILLIE"

Reddish-lavender; throat ruby mottled with white. Flowers large. Spikes well built.

$\begin{array}{lrccc} & \text { Bulbs } & \text { One-half Size } & \text { One-quarter Size } & \text { Bulblets } \\ \text { Per Bulb } & .15 & . .50 & \cdots & . .25 \\ \text { Per Doz. } & 1.00 & .50 & .95 & .25 \\ \text { Per 100 } & 7.50 & 3.75 & 1.90 & 1.00\end{array}$

No. 5-“ALOHA"

Ground color is a deep rosy pink with the tips of the petals shading into carmine. Ruby lip speckled with silver. Flowers are very large and well open. Extra fine variety.

$\begin{array}{lrccc} & \text { Bulbs } & \text { One-half Size } & \text { One-quarter Size } & \text { Bulblets } \\ \text { Per Bulb } & .50 & .25 & .93 & . .95 \\ \text { Per Doz. } & 5.00 & 2.50 & 1.25 & .75 \\ \text { Per 100 } & 40.00 & 20.00 & 10.00 & 5.00\end{array}$

No. 9- "SAUSALITO"

Rose pink overlaid with bright geranium pink. Magenta throat speckled with white. Flowers very large and heavily ruffled.

$\begin{array}{lrccc} & \text { Bulbs } & \text { One-half Size } & \text { One-quarter Size } & \text { Bulblets } \\ \text { Per Bulb } & .50 & .25 & .92 & . .95 \\ \text { Per Doz. } & 5.00 & 2.50 & 1.25 & .75 \\ \text { Per } 100 & 40.00 & 20.00 & 10.00 & 5.00\end{array}$

\section{No. 10-"ANNA EBERIUS"}

Deep Rhodamine Purple, shading into very deep Bordeaux center. This variety has created a sensation everywhere with growers and amateurs alike. We were so heavily sold out of this number last season that we camnot offer it. Withdrawn to increase stock.

\section{No. 13- "SUNSET"}

Pale rosy pink overlaid with cerise. Delicate canary yellow throat. Large blooms and very long spikes.

$\begin{array}{lrccc} & \text { Bulbs } & \text { One-half Size } & \text { One-quarter Size } & \text { Bulblets } \\ \text { Per Bulb } & .50 & .25 & .0 .5 & .0 .5 \\ \text { Per Doz. } & 5.00 & 2.50 & 1.25 & .000 \\ \text { Per } 100 & 40.00 & 20.00 & 10.00 & 5.00\end{array}$

\section{No. 19_"MYRTLE McNALLY"}

Cream with touches of rose pink on the edges. Ruby center with canary lip heavily ruffed. This is one of the best ruffled varieties ever put out.

\begin{tabular}{|c|c|c|c|c|}
\hline & Bulbs & One-half Size & One-quarter Size & Bulblets \\
\hline $\begin{array}{l}\text { Per Bulb } \\
\text { Per Doz. }\end{array}$ & 2.50 & 1.25 & .75 & $\ddot{.4} \dot{5}$ \\
\hline Per 100 & 20.00 & 10.00 & 5.00 & 2.50 \\
\hline
\end{tabular}


RICHARD DIENER CO., INC., KENTFIELD, MARIN COUNTY, CALIFORNIA

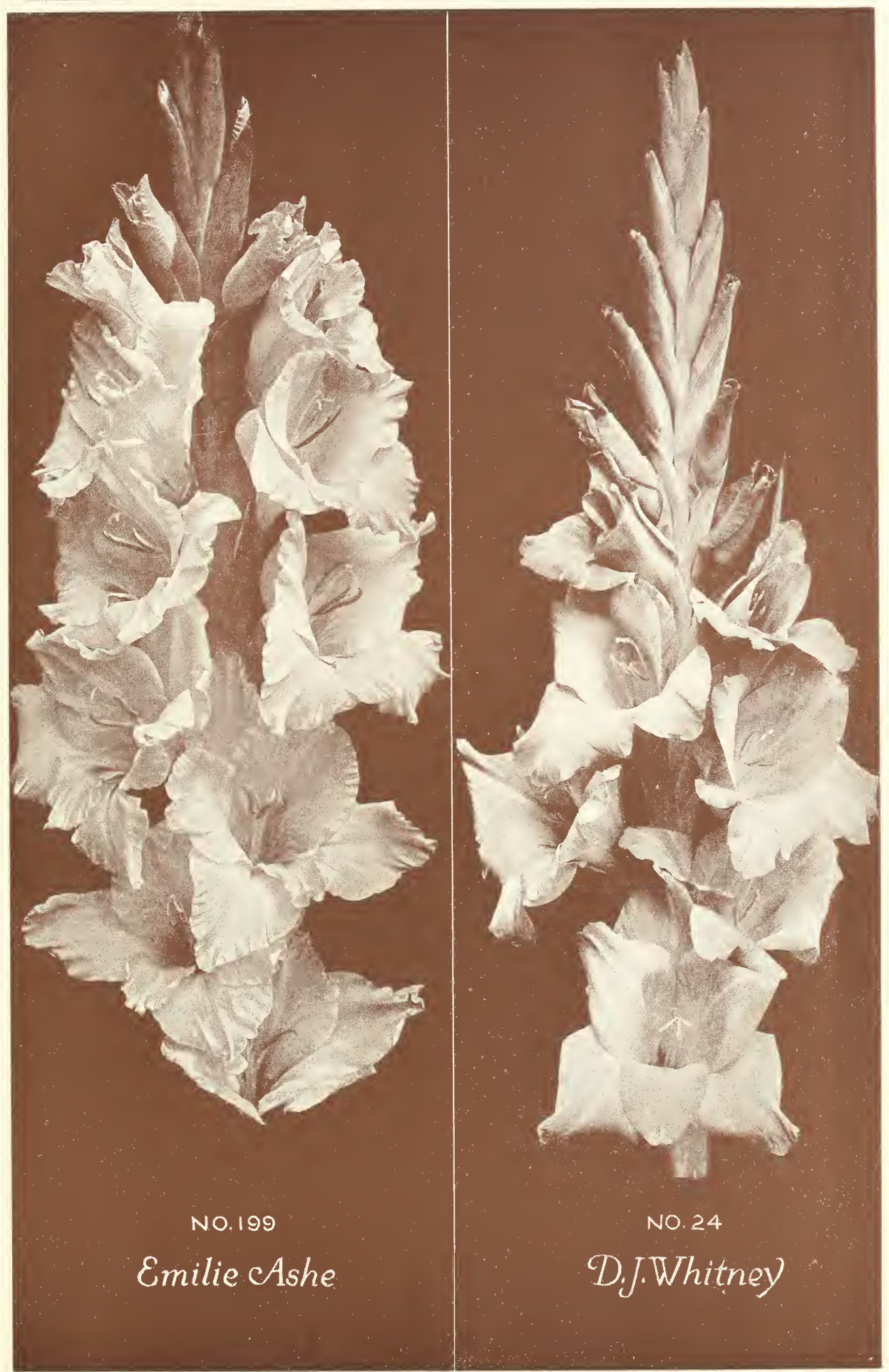




\section{No. 22-"SAN ANSELMO"}

Pure white delicately penciled with ruby pink. Flowers very large and free. Fine for cut flowers.

$\begin{array}{lrccc} & \text { Bulbs } & \text { One-half Size } & \text { One-quarter Size } & \text { Bulblets } \\ \text { Per Bulb } & .10 & .00 & . .9 & . .95 \\ \text { Per Doz. } & 1.00 & .60 & .00 & .25 \\ \text { Per } 100 & 8.00 & 4.00 & 2.00 & 1.00\end{array}$

\section{No. 23- "DIENER'S AMERICAN BEAUTY"}

Brilliant American Beauty color. Throat creamy yellow striped with ruby. One spike opening a large number of flowers at one time forms an immense bouquet. Flowers very large. Only one dozen bulbs to each customer.

$\begin{array}{lcccc} & \text { Bulbs } & \text { One-half Size } & \text { One-quarter Size } & \text { Bulblets } \\ \text { Per Bulb } & 1.00 & .50 & \ldots & \ldots \\ \text { Per Doz. } & 10.00 & 5.00 & \ldots & \ldots \\ \text { Per 100 } & \ldots & \ldots & \ldots & \ldots\end{array}$

\section{No. 24 "D. J. WHITNEY"}

Geranium pink with salmon and rose shadings. Lower petals lemon yellow with carmine, opens in a full cluster at the apex of the stem giving the appearance of a Canna. Earliest of all, good for forcing.

$\begin{array}{lcccc} & \text { Bulbs } & \text { One-half Size } & \text { One-quarter Size } & \text { Bulblets } \\ \text { Per Bulb } & 1.00 & .50 & 0.00 & . \dot{5} \\ \text { Per Doz. } & 10.00 & 5.00 & 2.00 & 10.00 \\ \text { Per } 100 & 80.00 & 40.00 & 20.00 & 10.00\end{array}$

\section{No. 31- "PRESIDENT C. C. MOORE"}

Begonia Rose striped with blackish red purple, also some spots of steel blue. Nice long, wiry spikes with large flowers well placed.

$\begin{array}{lrccc} & \text { Bulbs } & \text { One-half Size } & \text { One-quarter Size } & \text { Bulblets } \\ \text { Per Bulb } & .15 & . \dot{8} & . \dot{5} 0 & .00 \\ \text { Per Doz. } & 1.50 & 6.00 & 3.00 & .30 \\ \text { Per } 100 & 12.00 & 6.00\end{array}$

No. 32- "FAIRFAX"

Solid Magenta. Lower petals deep ruby circled with cream.

$\begin{array}{lcccc} & \text { Bulbs } & \text { One-half Size } & \text { One-quarter Size } & \text { Bulblets } \\ \text { Per Bulb } & .15 & . .85 & .9 \dot{0} & . .30 \\ \text { Per Doz. } & 1.50 & .8 .00 & .00 & 1.00 \\ \text { Per } 100 & 12.00 & 6.00 & 3.00 & \end{array}$

No. 38 - "JACK LONDON"

Light salmon with vermilion stripes. Canary yellow throat with ruby stripes. Our best commercial variety and largest propagator. It is perfect in every way. As a cut flower it is our most profitable variety, as we have never been able to supply the demand. It makes a wonderful showing under artificial light. We recommend everyone to plant as many bulbs of this variety as possible, as it surely is a most profitable investment. Even the smallest bulblet will bring a four foot spike five months after planting.

$\begin{array}{lrccc}\text { months after planting. } & \text { Bulbs } & \text { One-half Size } & \text { One-quarter Size } & \text { Bulblets } \\ \text { Per Bulb } & .25 & 1.25 & . .5 & . .45 \\ \text { Per Doz. } & 2.50 & 1.25 & .75 & .45 \\ \text { Per } 100 & 20.00 & 10.00 & 5.00 & 2.50\end{array}$

On larger amounts of bulbs and bulblets of this variety we can make a more reasonable figure.

No. 39_ "DR. LINCOLN COTHRAN"

Salmon pink overlaid with steel blue. Yellow lip. Fine form and bloom.

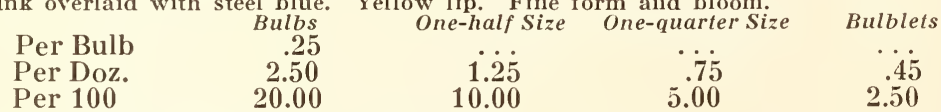

No. 50-"QUEEN OF THE NIGHT"

Deep maroon, almost black. Flowers very large and open.

\begin{tabular}{|c|c|c|c|c|}
\hline Per Bulb & $\begin{array}{l}\text { Bulbs } \\
5.00\end{array}$ & $\begin{array}{c}\text { One-half Size } \\
2.50\end{array}$ & $\begin{array}{c}\text { One-quarter Size } \\
1.25\end{array}$ & Bulblet \\
\hline Per Doz. & 50.00 & 25.00 & 12.50 & 6.25 \\
\hline Per 100 & $\cdots$ & $\cdots$ & $\ldots$ & \\
\hline
\end{tabular}

No. 67- "MRS. J. K. ARMSBY"

Coral pink, delicately penciled with vermilion near the edges. Throat cream speckled with ruby. Flowers very large, a great many opening at one time, and of great lasting quality. This is one of our finest productions and surely will satisfy any critic. All who reported on this praise it highly.

$\begin{array}{lrccc} & \text { Bulbs } & \text { One-half Size } & \text { One-quarter Size } & \text { Bulblets } \\ \text { Per Bulb } & 3.00 & 1.50 & .85 & .50 \\ \text { Per Doz. } & 30.00 & 15.00 & 7.50 & 3.75 \\ \text { Per } 100 & 240.00 & 120.00 & 60.00 & 25.00\end{array}$




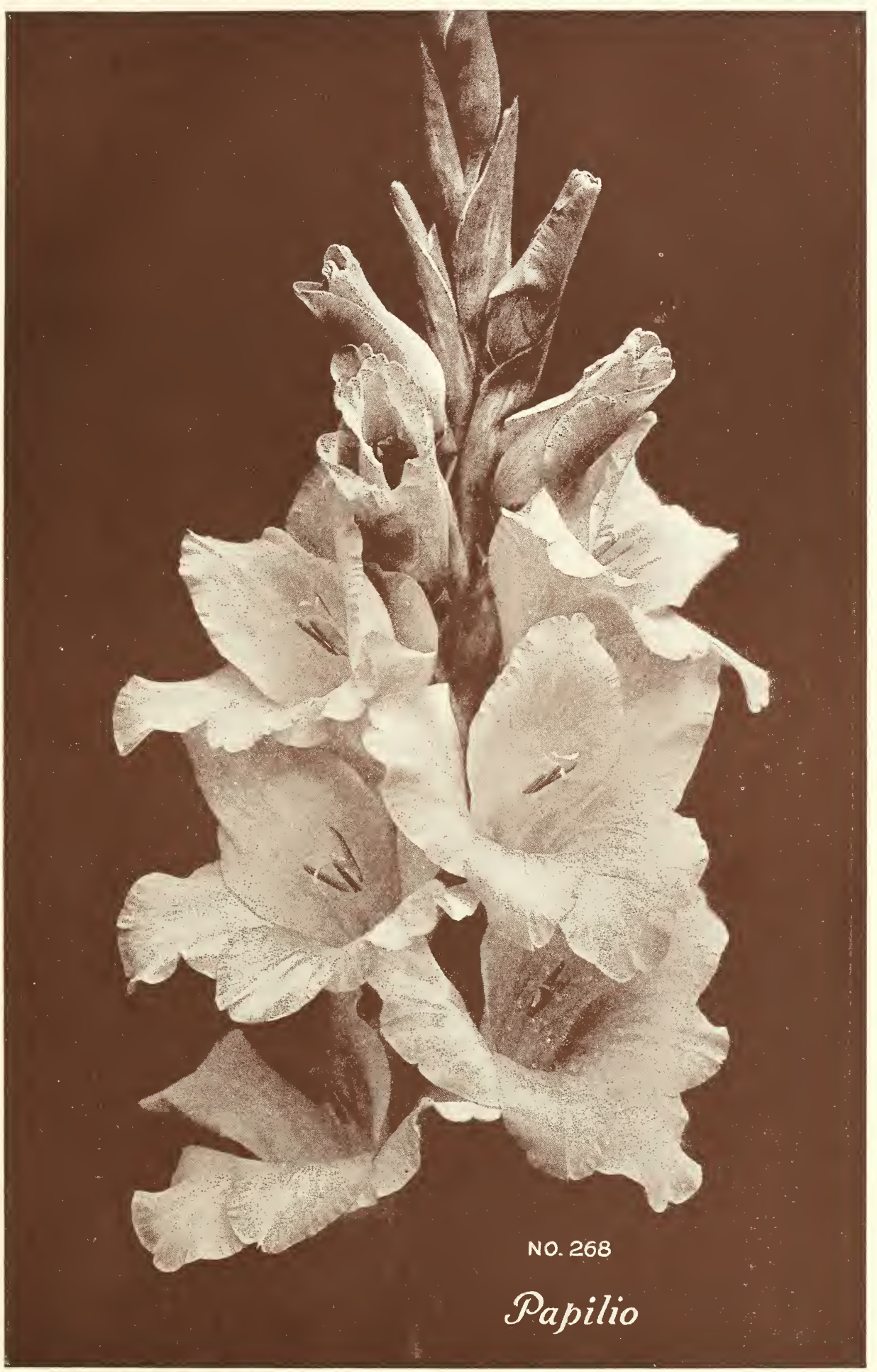


No. 70- "LILLIAN HARRIS COFFIN"

Shrimp pink overlaid with peach. Lower petals lemon yellow with dashes of ruby. Nice long spikes. Fine cut flower variety.

No. 82- "JOHN MERLE COULTER"

Rich scarlet red, almost black throat. Petals of velvety texture, flowers large and flaring. Beautiful variety.

$\begin{array}{lrrcc}\text { Per Bulb } & \text { Bulbs } & \text { One-half Size } & \text { One-quarter Size } & \text { Bulblets } \\ \text { Per Doz. } & 2.50 & 1.2 \dot{5} & .0 .75 & . .45 \\ \text { Per } 100 & 20.00 & 10.00 & 5.00 & 2.00\end{array}$

No. 83 "MRS WILLARD RICHARDSON"

Deep crimson, maroon center. Petals of very heavy texture. Large flaring blossoms. An unexcelled red which does not fade.

No. 89- "NORMA KRAUSGRILL"

Light rose pink with deep strawberry stripes. Lemon yellow splashes on lower petals covered with spots of ruby.
Per Pulb
Per Doz.
Bulbs
Per 100
25.00
One-half
1.00
12.50
200.00
100.00
$6 . \dot{2} \dot{j}$
50.00
Bulblets

No. 90 "CAROLINE FREAR BURKE"

Ground color, white, overlaid with rose pink. Brilliant ruby lip.

$\begin{array}{lrccc}\text { Per Pulb } & \text { Bulbs } & \text { One-lialf Size } & \text { One-quarter Size } & \text { Bulblets } \\ \text { Per Doz. } & .25 & 1.25 & .95 & .4 \dot{5} \\ \text { Per } 100 & 20.00 & 10.00 & 5.00 & .45\end{array}$

No. 96-"RICHARD DIENER"

Pure geranium pink with a slight sprinkling of ruby on creamy yellow center. Large flaring blossoms rivaling an orchid in beauty. The acne of perfection in coloring. This variety has created a sensation wherever shown.

$\begin{array}{lcccc} & \text { Bulbs } & \text { One-half Size } & \text { Onc-quarter Size } & \text { Bnlblets } \\ \text { Per Bulb } & 5.00 & 2.50 & 1.25 & .75 \\ \text { Per Doz. } & 50.00 & 25.00 & 12.50 & 6.25 \\ \text { Per } 100 & 400.00 & 200.00 & 100.00 & 40.00\end{array}$

No. 98-"MABLE HUBBARD"

Pure white. Lower petals tinged with pale sulphur yellow, covered with a violet spot. Very fine spikes.

\begin{tabular}{lr} 
Per Bulb & Bulbs \\
Per Doz. & .50 \\
Per 100 & 50.00 \\
\hline
\end{tabular}

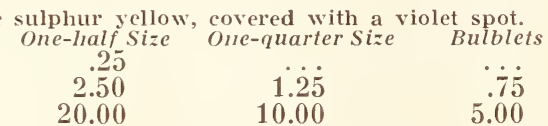

No. 101_"ELSE ROSE"

Light mallow pink overlaid with light ruby. Creamy yellow throat. Orchid flowering. Extra fine

$\begin{array}{lrrrr} & \text { Bulhs } & \text { One-half Size } & \text { One-quarter Size } & \text { Bulblets } \\ \text { Per Bulb } & .75 & .40 & 1.90 & 1.00 \\ \text { Per Doz. } & 7.50 & 3.75 & 1.90 & 7.00 \\ \text { Per 100 } & 60.00 & 30.00 & 15.00 & 7.50\end{array}$

No. 102- "INA COLLINS"

Tyrian pink, striped and spotted with cerise and deep lilac. Lip has a circle of primrose edged with light ruby. Orchid flowering.

$\begin{array}{lrrrr} & \text { Bulhe } & \text { One-half Size } & \text { One-quarter Size } & \text { Bulblets } \\ \text { Per Bulb } & .50 & .25 & .25 & . .75 \\ \text { Per Doz. } & 5.00 & 2.50 & 1.25 & .75 \\ \text { Per 100 } & 40.00 & 20.00 & 10.00 & 5.00\end{array}$

No. 107- "HEART OF JUANITA"

Deep cherry scarlet. Crimson and white throat. A very beautiful variety which does not fade in the sunlight. One of the best dark colored varieties.

$\begin{array}{lcccc} & \text { Bulbs } & \text { One-half Size } & \text { One-quarter Size } & \text { Bulblets } \\ \text { Per Bulb } & .50 & .25 & .0 .5 & .5 \dot{5} \\ \text { Per Doz. } & 5.00 & 2.50 & 1.25 & .75 \\ \text { Per } 100 & 40.00 & 20.00 & 10.00 & 5.00\end{array}$

No. 113_"MRS. NEWELL VANDERBILT"

Light salmon pink, heavily ruffed. Throat sulphur yellow, mottled and striped with crimson. Large flowers, clean grower and A-1 variety. Plant all you can of this varicty.

$\begin{array}{lcccc} & \text { Bulbs } & \text { One-half Size } & \text { One-quarter Size } & \text { Bulblets } \\ \text { Per Bulb } & 1.00 & .50 & .0 . \dot{0} & 1.2 j \\ \text { Per Doz. } & 10.00 & 5.00 & 2.50 & 8.00 \\ \text { Per } 100 & 80.00 & 40.00 & 20.00 & \end{array}$




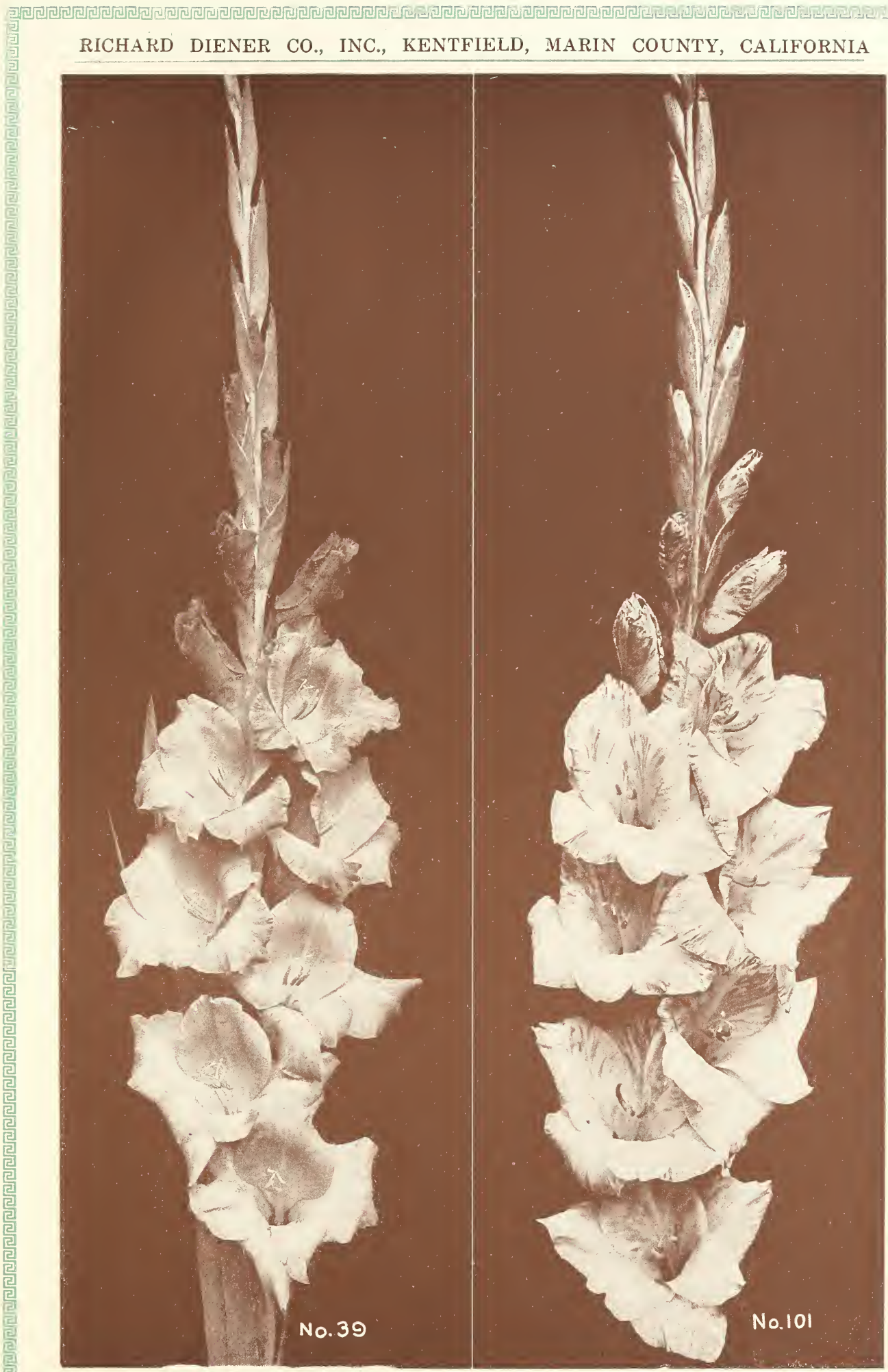




\section{No. 117- "SIERRA"}

Cream, speckled and striped with rose and lilac. Yellow and ruby lip. Flowers large and very heavily ruflled. Strong spikes.

\begin{tabular}{|c|c|c|c|c|}
\hline Per Bulb & $\begin{array}{r}\text { Bulbs } \\
1.00\end{array}$ & One-half Size & One-quarter Size & Bulblets \\
\hline Per Doz. & 10.00 & 5.00 & $2 \dot{5} \dot{0}$ & 1. \\
\hline Per 100 & 80.00 & 40.00 & 20.00 & 8.00 \\
\hline
\end{tabular}

\section{No. 120 - "MRS. STANLEIGH ARNOLD"}

Soft phlox pink, with violet and liseran purple shadings. Lip has markings of Chinese violet. One of the most exquisite colors so far produced in Gladioli. Well formed spikes. As our stock is limited, we cannot offer this in quantity.

$\begin{array}{lcccc} & \text { Bulbs } & \text { One-half Size } & \text { One-quarter Size } & \text { Bulblets } \\ \text { Per Bulb } & 10.00 & 5.00 & 2.50 & 1.25 \\ \text { Per Doz. } & \ldots & \ldots & \ldots & \ldots \\ \text { Per 100 } & \ldots & \ldots & \ldots & \ldots\end{array}$

\section{No. 130 - "THOMAS T. KENT"}

Shell pink overlaid with strawberry. Crimson streak on each petal. Very vigorous in growth; this is one of our largest varieties and one of the heaviest propagators. Spikes very tall. It has proven a favorite everywhere.

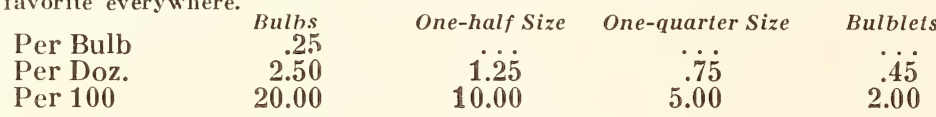

No. 136-"ADELINE KENT"

Delicate light rose pink, heavily ruffled from edges to center. Orchid coloring ranging from flesls to new rose on the outer edges. Throat straw color with ruby dashes. This is one of the most beautiful rufled Gladiolus in existence. The flowers are large and appear on fine long spikes.

\begin{tabular}{|c|c|c|c|c|}
\hline & & & $e-q u a r t$ & \\
\hline Per Bulb & 1.00 & $\cdots$ & $\cdots$ & \\
\hline Per Doz. & 10.00 & $\cdots$ & $\cdots$ & $\cdots$ \\
\hline $\begin{array}{l}\text { Per } 100 \\
\text { ore than } 6\end{array}$ & tom & as our stock is & limited. & 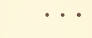 \\
\hline
\end{tabular}

No more than 6 bulbs to a customer, as our stock is limited.

No. 145-"MRS. CO'THRAN"

Corinthian Red and Ashes of Roses. Very iridescent. An odd color. Fine long spikes.

\begin{tabular}{lrccc} 
Per Bulb & Bulbs & One-half Size & One-quarter Size & $\begin{array}{c}\text { Bulblets } \\
\text { Per Doz. }\end{array}$ \\
Per 100 & 2.250 & $1.2 \dot{5}$ &.$\dot{7}$ &. .5 \\
\hline
\end{tabular}

No. 162-"MARY ROBERTS RINEHART"

Very pale Chalcedony yellow overlaid with light mallow purple. Lip lemon with a splash of ruby shading into violet.

$\begin{array}{lrccc}\text { Per Bulb } & \text { Bulbs } & \text { One-half Size } & \text { One-quarter Size } & \text { Bulblets } \\ \text { Per Doz. } & .20 & .0 .00 & .0 \dot{6} & . \dot{3} \dot{5} \\ \text { Per } 100 & 2.00 & 1.00 & \ldots & \ldots\end{array}$

No. 164 "CAPTAIN ASHER CARTER BAKER"

Rich velvety red with darker center. Large flowers. This variety was awarded the silver medal at the P. P. I. E., 1915, for the best of all the red Gladioli shown.

\begin{tabular}{|c|c|c|c|}
\hline Per Bulb & .05 & $\ldots$ & One-quarter Size \\
\hline Per Doz. & .50 & $\ddot{3} \dot{5}$ & $\ddot{2} \dot{5}$ \\
\hline Per 100 & 3.50 & 1.75 & 1.00 \\
\hline Per 1000 & 30.00 & 15.00 & 7.50 \\
\hline
\end{tabular}

No. 165-"DR. FREDERICK J. V. SKIFF"

Clear, flesh pink, light ruby center; sometimes slightly striped with rose pink; strong, vigorous growth, abundant foliage; one bulb will very often bring up to 130 perfect flowers 6 inches in diameter. Awarded Gold Medal at P. P. I. E., 1915.

Withdrawn to increase stock.

\section{No. 166- "DIENER'S WHITE"}

Milk white with a faint touch of pink in the throat. Lower petals overlaid with pale orclid. Flowers extra large. Awarded Gold Medal at P. P. I. E., 1915.

\begin{tabular}{|c|c|c|c|c|}
\hline Per Bulb & Bulbs & alf Size & One-quarter Size & Bulblets \\
\hline Per Doz. & 10.00 & 5.00 & $\dot{2} \dot{50} 0$ & 1.2 \\
\hline Per 100 & 80.00 & 40.00 & 20.00 & 10.00 \\
\hline
\end{tabular}

No. 173-"HAWAII"

Rose pink fading into a lighter center. Lower petals of crimson outlined with white. Clean grower, elegant spikes, flowers large.

$\begin{array}{lrrrr} & \text { Bulbs } & \text { One-half Size } & \text { One-quarter Size } & \text { Bulblets } \\ \text { Per Bulb } & .50 & .25 & . \dot{2} & . \dot{5} \\ \text { Per Doz. } & 5.00 & 2.50 & 1.25 & 5.00 \\ \text { Per 100 } & 40.00 & 20.00 & 10.00 & 50\end{array}$


RICHARD DIENER CO., INC., KENTFIELD, MARIN COUNTY, CALIFORNIA

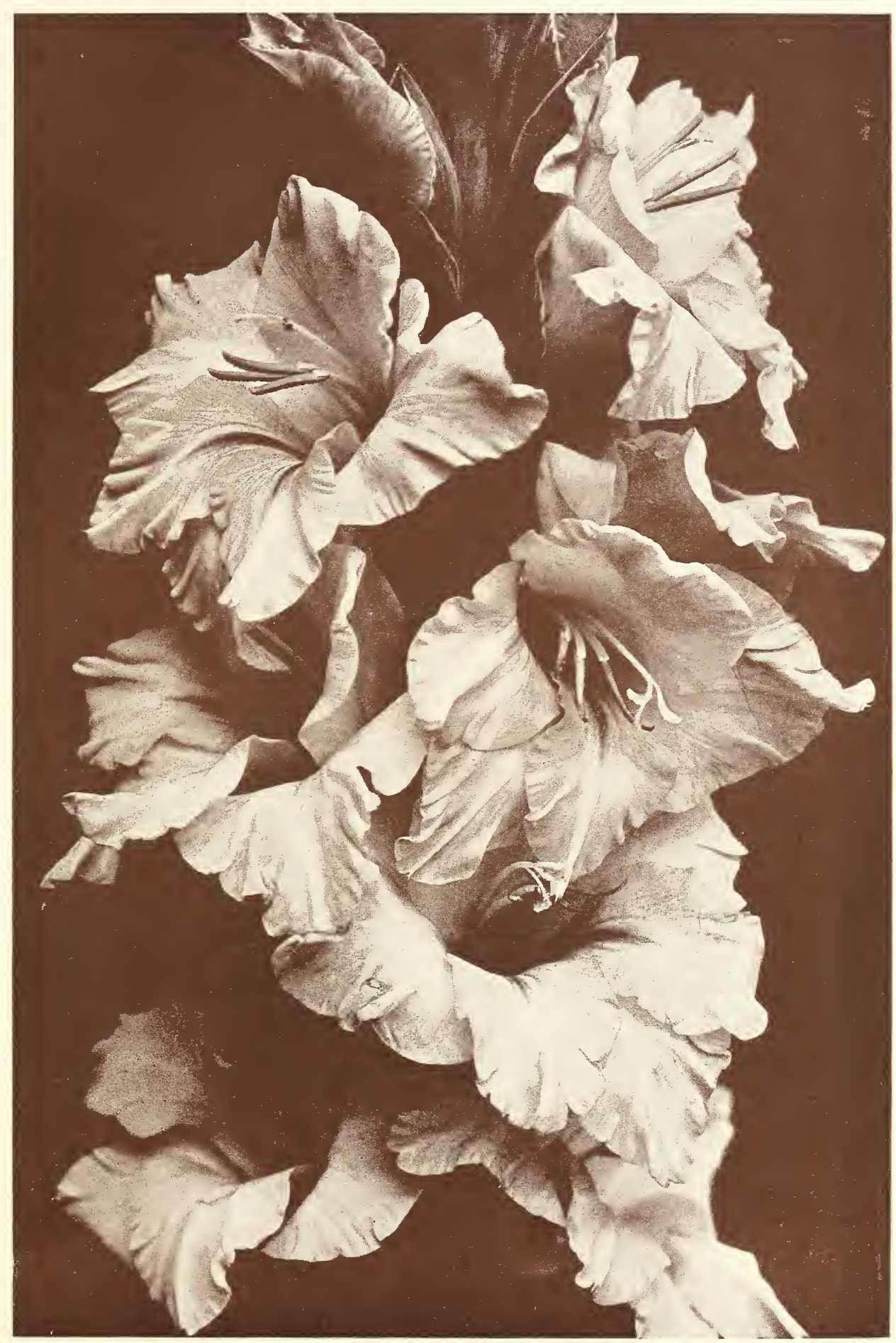

No. 117-SIERRA

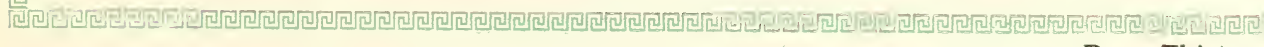




\section{No. 174_-"ATLANTIS"}

Geranium pink" overlaid with vermilion. White eenter with huge spots of scarlet with a deep crimson stripe through the lip. Very large flowers and when open are alnost flat like Amaryllis. Long spikes.

$\begin{array}{lcccc} & \text { Bulbs } & \text { One-half Size } & \text { One-quarter Size } & \text { Bulblets } \\ \text { Per Bulb } & 1.00 & .50 & .25 & . \dot{5} \\ \text { Per Doz. } & 10.00 & 5.00 & 2.50 & 1.25 \\ \text { Per } 100 & 80.00 & 40.00 & 20.00 & 8.00\end{array}$

No. 175-"MRS LEON DOUGLAS"

Ground eolor Begonia Rose, striped with flame and brilliant searlet. Lip is pale lemon slightly speckled with ruby. The size of the flowers, .we believe, is the largest in existence, and all are very open and well set on the long spikes. The spikes are extra long and have up to as many as seven side spikes on each nıain spike. The bulbs are of immense size. This is one of the unost beautiful varieties ever produeed.

$\begin{array}{lrccc} & \text { Bulbs } & \text { One-half Size } & \text { One-quarter Size } & \text { Bulblets } \\ \text { Per Bulb } & 3.00 & 1.50 & .85 & .50 \\ \text { Per Doz. } & 30.00 & 15.00 & 7.50 & 3.75 \\ \text { Per } 100 & 240.00 & 120.00 & 60.00 & 25.00\end{array}$

No. 176 "MISS MAUD FAY",

Pale Amaranth pink with a light stripe running through the eenter of eaeh petal. The edges of the petals shade into Tyrian pink. Flow ers are very open and large. The spikes are never straight but curve into various direetions.

$\begin{array}{lrrrr}\text { into various direetions. } & \text { Bulhs } & \text { One-half Size } & \text { One-quarter Size } & \text { Bulblet.s } \\ \text { Per Bulb } & .50 & .25 & . .5 & . .75 \\ \text { Per Doz. } & 5.00 & 2.50 & 1.25 & .700 \\ \text { Per } 100 & 40.00 & 20.00 & 10.00 & 5.00\end{array}$

\section{No. 177- "JOSEPH FIELD"}

Dawn pink, lightly striped with mallow pink and ruby. Lip has stripe of eream white. Fine, long, well built spikes. Flowers of very large size. First class show and eut variety.

$\begin{array}{lrccc} & \text { Bulbs } & \text { One-half Size } & \text { One-quarter Size } & \text { Bulblets } \\ \text { Per Bulb } & 10.00 & 5.00 & 2.50 & 1.25 \\ \text { Per Doz. } & 100.00 & 50.00 & 25.00 & 12.50 \\ \text { Per } 100 & 800.00 & 400.00 & 200.00 & 80.00\end{array}$

No. 180-"MRS. TRLXTON BEALE"

Cream, rose pink striped, very large open flowers. Lip yellow and brown sprinkled. Fine long well built spikes. A beauty.

$\begin{array}{lrccc} & \text { Bulbs } & \text { One-halfSize } & \text { One-quarter Size } & \text { Bulblets } \\ \text { Per Bulb } & .50 & .25 & 1.25 & . .5 j \\ \text { Per Doz. } & 5.00 & 2.50 & 10.00 & 5.00 \\ \text { Per } 100 & 40.00 & 20.00 & 10.00 & 5.00\end{array}$

\o. 181 "ALMA"

Hermosa pink blotehed with mallow purple. Lip sulphur yellow striped with ruby. Very heavily ruffled. Yery large flowers on long well built spikes.

$\begin{array}{lcccc} & \text { Bulbs } & \begin{array}{c}\text { One-half Size } \\ \text { One-quarter Size }\end{array} & \text { Bulblets } \\ \text { Per Bulb } & 5.00 & 2.50 & 1.25 & .75 \\ \text { Per Doz. } & 50.00 & 25.00 & 12.50 & 6.25 \\ \text { Per } 100 & 400.00 & 200.00 & 100.00 & 50.00\end{array}$

No. 186 - "LILLIAN WEBB"

Strawberry pink with earmine velvety eenters, overlaid with maroon and brownish stripes. The stems are slender and give the appearance of a lily

$\begin{array}{lcccc} & \text { Bulbs } & \text { One-haif Size } & \text { One-quarter Size } & \text { Bulblets } \\ \text { Per Bulb } & .05 & . .5 & . .35 & .20 \\ \text { Per Doz } & .50 & .35 & .250 & .50 \\ \text { Per } 100 & 4.00 & 2.00 & 1.00 & .50\end{array}$

ㄱo. 187- "COSTA RICA"

Clear orehid, ruffled edges tinged with deeper eolor. Crimson eenter. Large fine spikes. A very valuable variety.

\begin{tabular}{|c|c|c|c|c|}
\hline Don Pulb & Bulbs & One-half Size & One-quarler Size. & Bulblet \\
\hline $\begin{array}{l}\text { Per Bulb } \\
\text { Per Doz. }\end{array}$ & 2.50 & 125 & $\ddot{7} \dot{5}$ & . \\
\hline Per 100 & 20.00 & 10.00 & 5.00 & 2.00 \\
\hline
\end{tabular}

No. 188- "MRS. MARY STEARNS BLRKE"

Canary yellow, overlaid with aprieot and tinged with rose. Deep canary eenter with ruby throat. Long spikes with flowers symmetrieally plaeed; stem wiry and upright, flowers large. It is one of the finest yellows known so far. When known it will be one of the most grown varieties for cut flowers.

\begin{tabular}{|c|c|c|c|c|}
\hline Per Bulb & Bulbs & One-half Size & One-quarter Size & Bulblets \\
\hline Per Doz. & 5.00 & 2.50 & $1 . \dot{2} \dot{5}$ & $\ddot{7}$ \\
\hline Per 100 & 40.00 & 20.00 & 10.00 & 5.00 \\
\hline
\end{tabular}


RICHARD DIENER CO., INC., KENTFIELD, MARIN COUNTY, CALIFORNIA

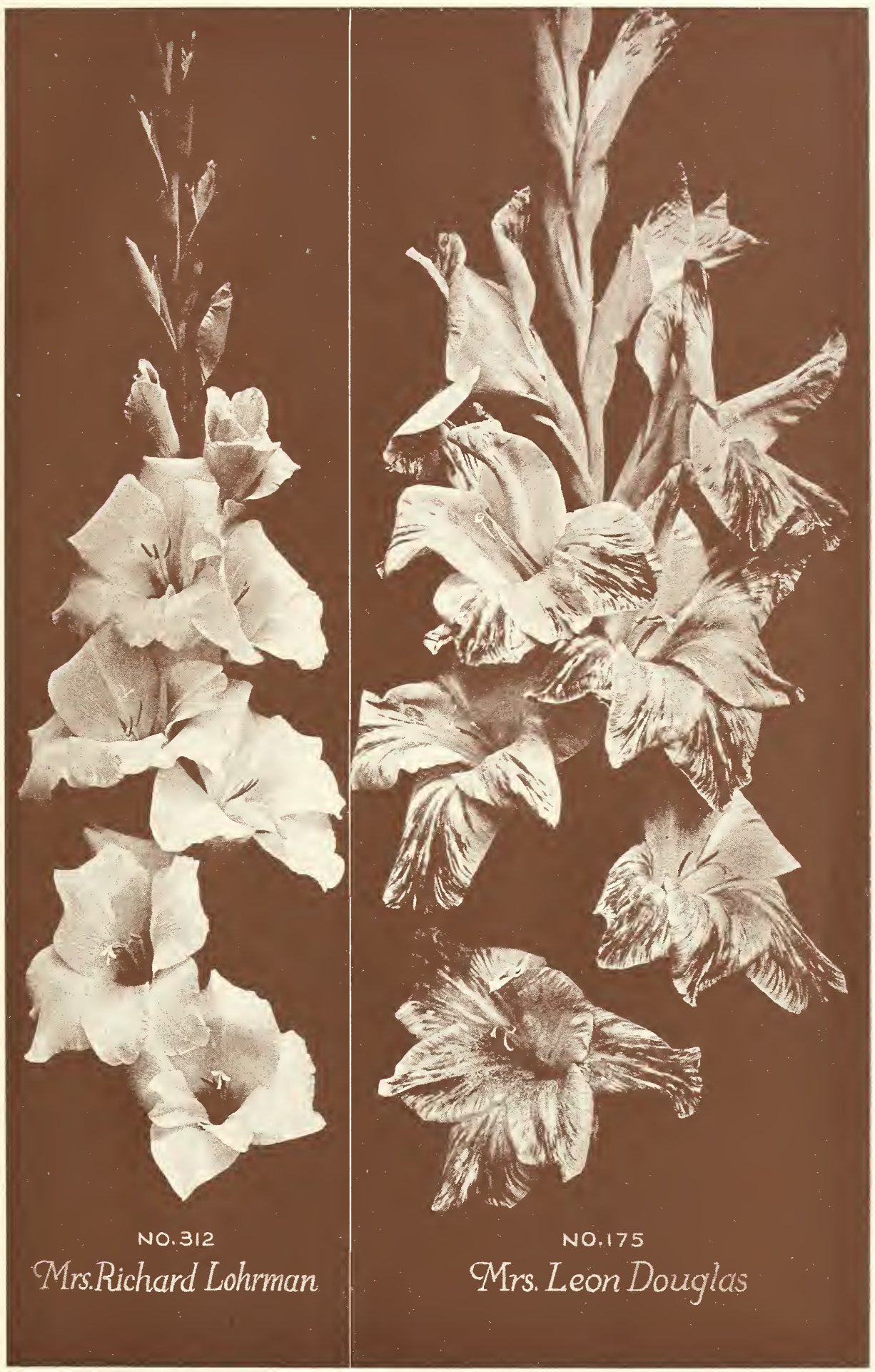




\section{No. 189 -ELIZABETH GERBERDING"}

Shell pink, heavily ruffed, shading into róse on the outer edges. Center pale canary and ruby. Spikes large and full.

$\begin{array}{lrccc}\text { Per Bulb } & \text { Bulbs } & \text { One-half Size } & \text { One-quarter Size } & \text { Bulblets } \\ \text { Per Doz. } & .10 & . .50 & . .35 & . .25 \\ \text { Per } 100 & 1.00 & . .75 & .350 & 1.00\end{array}$

No. 190- "ALTAMYRA"

Lilac pink, striped with mallow purple. White spotted lip. Flowers of the very largest size.

$\begin{array}{lrrrr} & \text { Bulbs } & \text { One-half Size } & \text { One-quarter Size } & \text { Bulblet } \\ \text { Per Bulb } & 2.00 & 1.00 & .50 & 2.50 \\ \text { Per Doz. } & 20.00 & 10.00 & 5.00 & 2.00 \\ \text { Per 100 } & 160.00 & 80.00 & 40.00 & 20.00\end{array}$

No. 191-"MRS. H. E. BOTHIN"

Light geranium pink, heavily ruffled, flame scarlet center. Strong, well built spikes, first class show and cut variety. One of the loveliest combinations in Gladioli. It has created a sensation wherever exhibited. One of the greatest and most perfect creations ever produced. A heavy propagator. We recommend to procure as many bulbs of this variety as possible.

$\begin{array}{lrccc} & \text { Bulbs } & \text { One-half Size } & \text { One-quarter Size } & \text { Bulblet } \\ \text { Per Bulb } & .25 & . \dot{2} & . \dot{5} & . . \dot{5} \\ \text { Per Doz. } & 2.50 & 1.25 & 5.00 & 2.50\end{array}$

\section{No. 192-"VICTORY"}

Carmine, shading into lighter tone in the center. Lip mottled with white. Flowers are very large; immense long spikes; one of our largest reds.

$\begin{array}{lcccc} & \text { Bulbs } & \text { One-half Size } & \text { One-quarter Size } & \text { Bulblets } \\ \text { Per Bulb } & 1.00 & .50 & \dot{0} \dot{0} & 1 . \dot{2} \dot{j} \\ \text { Per Doz. } & 10.00 & 5.00 & 2.00 & 10.00 \\ \text { Per } 100 & 80.00 & 40.00 & 20.00 & \end{array}$

\section{No. 193-"JONATHAN WEBB"}

Light scarlet, a finely penciled white and ruby stripe running through each petal. Ruby mottled center. Very showy variety; makes elegant lily-like spike with wide open flowers.

Withdrawn to increase stock.

\section{No. 196-“"HELEN TODD"}

Amaranth pink with light stripes through the centers of the three upper petals. Ruby lip shading into deeper ruby at the throat. Rosy colored seam around the entire edge of the flower.

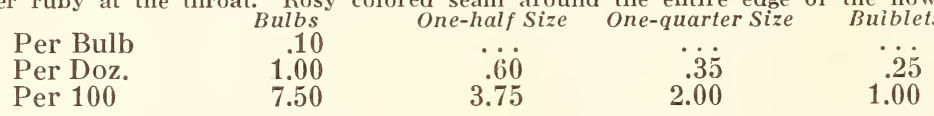

No. 198-"ROSS VALLEY"

Salmon pink delicately overlaid with mauve, giving ashes of roses effect. Red peacock eye in the center.

\begin{tabular}{lr} 
Per Bulb & Bulbs \\
Per Doz. & .10 \\
Per 100 & 1.00 \\
\hline
\end{tabular}
One-half Siz Bulblets

No. 199_"EMILE ASHE"

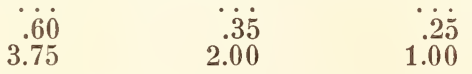

Snow white petals. Small cerise blotches on lower petals. Edges heavily ruffed. This is one of the best ruffed varieties ever put out.

\begin{tabular}{|c|c|c|c|}
\hline Per Bulb & $\begin{array}{c}\text { Bulbs } \\
.50\end{array}$ & $\begin{array}{c}\text { One-half Size } \\
.25\end{array}$ & One-quarter Size \\
\hline Per Doz. & 5.00 & 2.50 & $1 . \dot{2} \dot{5}$ \\
\hline Per 100 & 40.00 & 20.00 & 10.00 \\
\hline
\end{tabular}

No. 200-"MR. H. A. HYDE"

Snow white with blush pink trace throughout. Fine built spikes. Excellent cut flower varicty.

$\begin{array}{lcccc} & \text { Bulbs } & \text { One-half Size } & \text { One-quarter Size } & \text { Bulblet } \\ \text { Per Bulb } & 1.00 & .50 & .25 & .00 \\ \text { Per Doz. } & 10.00 & 5.00 & 2.50 & 1.25 \\ \text { Per } 100 & 80.00 & 40.00 & 20.00 & 10.00\end{array}$

No. 202_"MRS. JOHN R. WALSH"

Heavily ruffled, flesh pink. Three lower petals of flame scarlet shading into a dark ruby at the throat. Flowers are very large. This is a seedling of Mrs. Pendleton, Jr. It is a magnificent variety that should be in every collection.

$\begin{array}{lcccc} & & \\ \text { Per Bulb } & \text { Bulbs } & \text { One-half Size } & \text { One-quarter Size } & \text { Bulblets } \\ \text { Per Doz. } & 1.00 & .50 & .25 & .35 \\ \text { Per 100 } & 10.00 & 5.00 & 2.50 & 1.25 \\ & 80.00 & 40.00 & 20.00 & 8.00\end{array}$




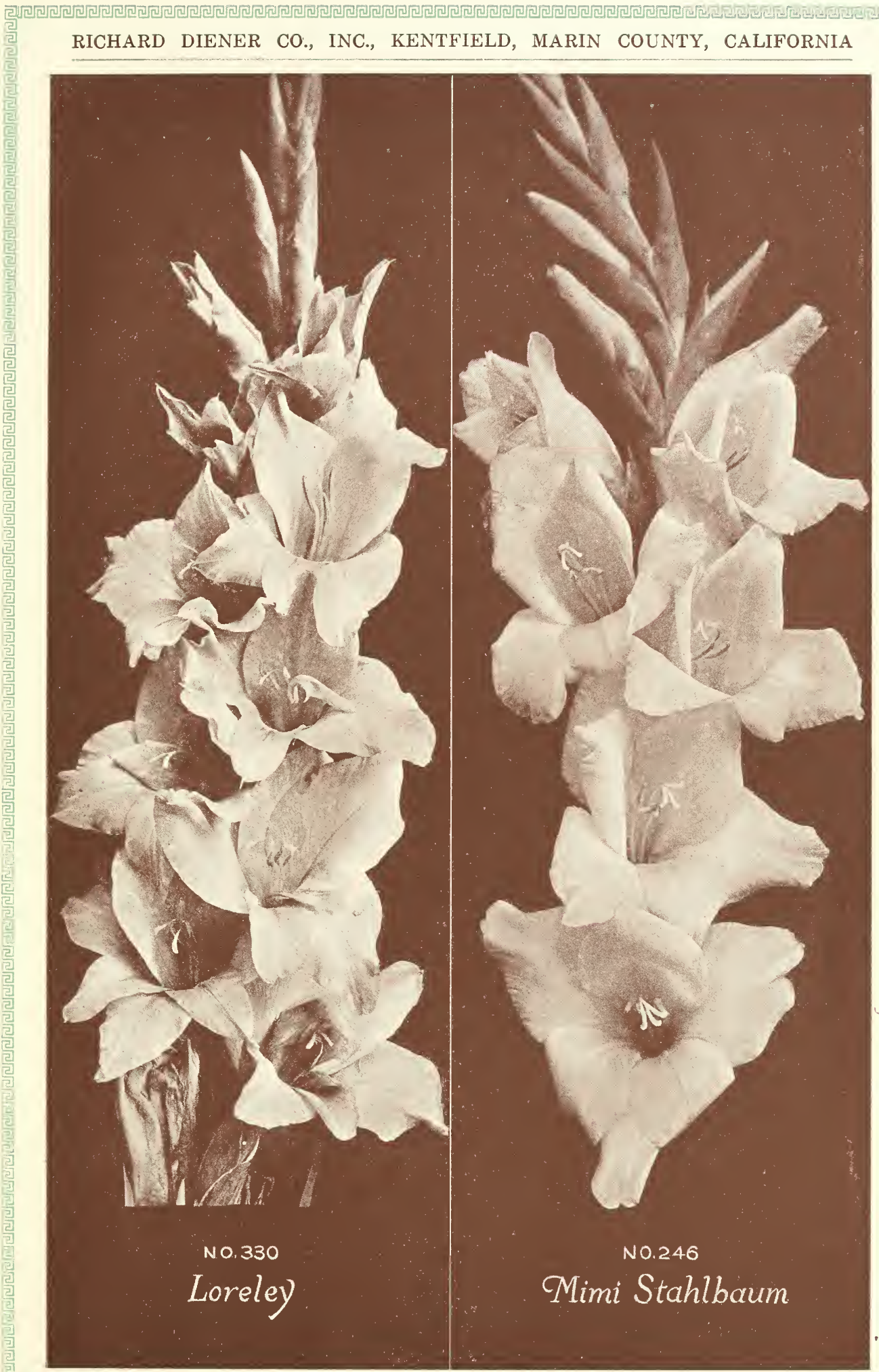




\section{No. 203-"SIR ROGER CASEMENT"}

ox-blood red, heavily ruffled. Dark maroon stripe through the center of the lip. of extremely velvety texture.

Dark
Bulbs

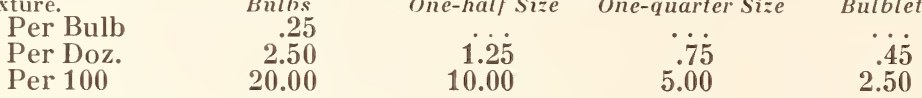

No. 204 -FRANK J. SYMMES"

Heavily ruffled peach red, with the three lower petals shading into a flame color and finally into a deep crimson. The lip has small spots of silver near the throat. This variety should be in every collection.

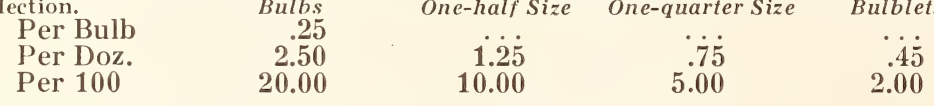

No. 205-"DA VID STARR JORDAN"

Immense flame colored, overlaid with scarlet. Lip mottled with crcam. FIowcrs very open like immense Amaryllis, with fine, wiry upright stems.

\begin{tabular}{|c|c|c|c|c|}
\hline Per Bulb & .25 & One-half Size & One-quarter Size & Bulblet \\
\hline Per Doz. & 2.50 & $1 . \dot{25}$ & .75 & .42 \\
\hline Per 100 & 20.00 & 10.00 & 5.00 & 2.00 \\
\hline
\end{tabular}

No. 206-"WILLIAM KENT"

Creamy shell pink with golden ochre center. Flowers very large. Declared by the biggest experts in Gladioli to be the best rumed Gladiolus yet produccd, considering size and amount of flowers open at one time. Fifteen open flowers at once is no rarity, while most of the ruffled varieties have only 3 to 4 open flowers at one time.

$\begin{array}{lcccc} & & & \\ \text { Per Bulb } & \text { Bulbs } & \text { One-half Size } & \text { One-quarter Size } & \text { Bulblets } \\ \text { Per Doz. } & 5.00 & 2.50 & 1.25 & .75 \\ \text { Per 100 } & 50.00 & 25.00 & 12.50 & 6.25 \\ & 400.00 & 200.00 & 100.00 & 50.00\end{array}$

\section{No. 209-"GENEVIEVE BOTHIN"}

Ground color Alizarine pink overlaid with Vandyke red shading into deep plum color. Tips of petals penciled with steel blue. Very odd. Flowers of large size.

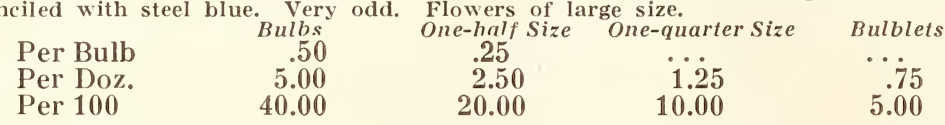

No. 211-"ZOE"

Deep magenta, velvet maroon center. Very large flowers. Exceptionally fine color.

$\begin{array}{lrrcc} & \text { Bulbs } & \text { One-half Size } & \text { One-quarter Size } & \text { Bulblets } \\ \text { Per Bulb } & 3.00 & 1.50 & .75 & . .55 \\ \text { Per Doz. } & 30.00 & 15.00 & 7.50 & 3.75 \\ \text { Per 100 } & 240.00 & 120.00 & 60.00 & 30.00\end{array}$

No. 213-"MRS. LILLIAN GUERNSEY"

Light buff yellow spotted with pink. Canary yellow center. Ruffed edgcs. Flowers very large. Fine long spikes. A-1 variety.

is our stock is limited we cannot offer the same in quantity.

$$
\begin{array}{cccccc} 
& \text { Bulbs } & \text { One-half Size } & \text { One-quarter Size } & \text { Bulblets }
\end{array}
$$

No. 216-"MARTHA FERNEKES"

lris blue, with large violet spot on lower pctals. Odd and beautiful variety admired by everyone.

$\begin{array}{lcccc} & \text { Bulbs } & \begin{array}{c}\text { One-half } \\ \text { Per Bulbe }\end{array} & \text { One-quarter Size } & \text { Bulblets } \\ \text { Per Doz. } & 1.00 & .50 & .25 & .5 \\ \text { Per 100 } & 10.00 & 5.00 & 2.50 & 1.25 \\ & 80.00 & 40.00 & 20.00 & 10.00\end{array}$

No. 218-"YOSEMITE"

Geranium red shading into Rose Doree. Light stripe through the center of each petal. Lip pale lemon yellow. Flowers very large and open. Amaryllis like. Spikes often reach seven feet high.

$\begin{array}{lcccc} & \text { Bulbs } & \text { One-half Size } & \text { One-quarter Size } & \text { Bulblet } \\ \text { Per Bulb } & 1.00 & .50 & .25 & . \dot{5} \\ \text { Per Doz } & 10.00 & 5.00 & 2.50 & 1.25 \\ \text { Per } 100 & 80.00 & 40.00 & 20.00 & 10.00\end{array}$

No. 220_ "ELDIVA"

Cerise with a lighter stripe running through the center. Penciled with crimson. Long fine spikcs. First class show variety.

$\begin{array}{lrccc} & \text { Bulbs } & \text { One-half Size } & \text { One-quarter Size } & \text { Bulblets } \\ \text { Per Bulb } & 4.00 & 2.00 & 1.00 & .60 \\ \text { Per Doz. } & 40.00 & 20.00 & 10.00 & 5.00 \\ \text { Per 100 } & 320.00 & 160.00 & 80.00 & 40.00\end{array}$


RICHARD DIENER CO., INC., KENTFIELD, MARIN COUNTY, CALIFORNIA
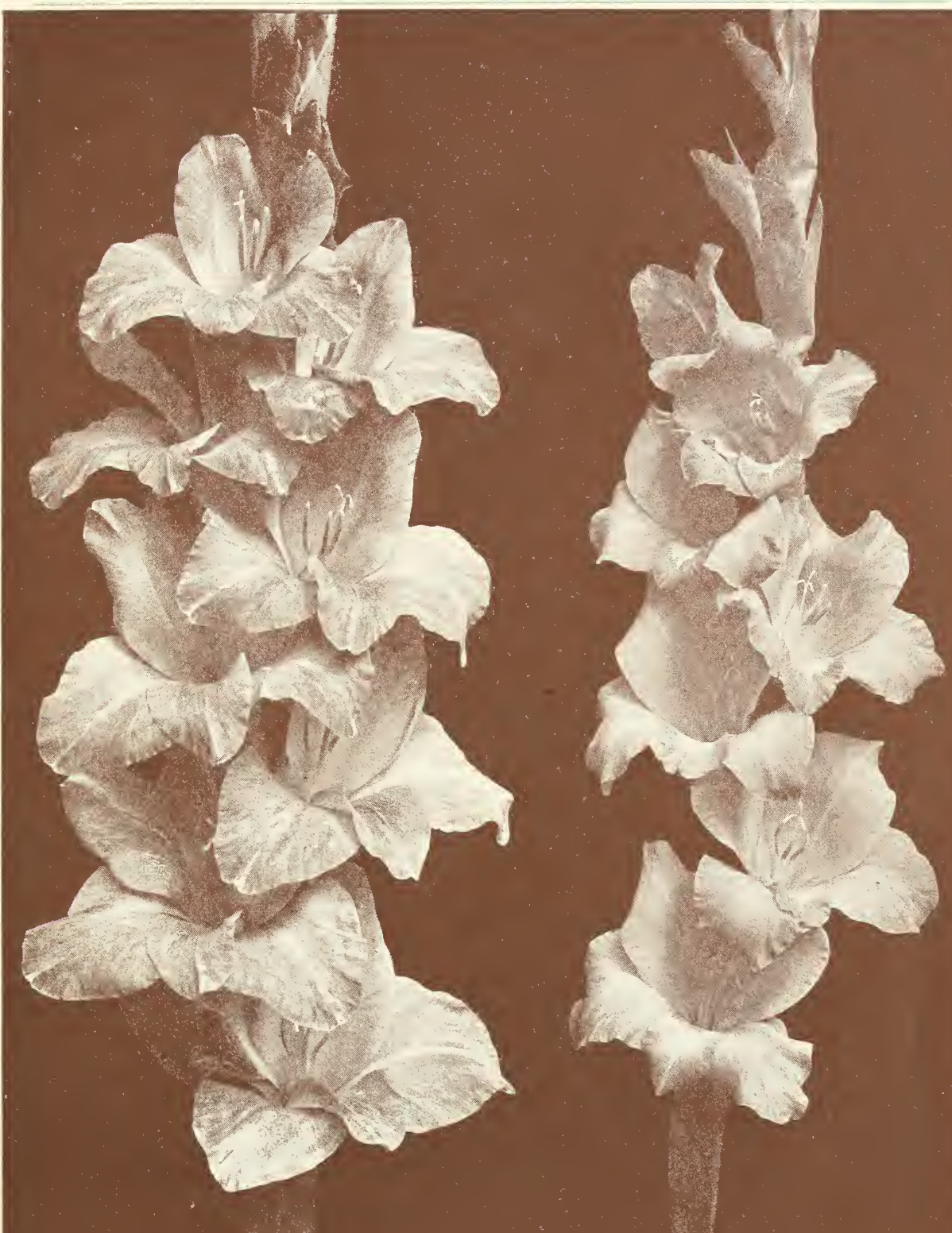

NO 266

NO. 145

Caroline Esberg

Mrs. Cothran 


\section{No. 221- "LONDON SMOKE"}

Corinthian red shading into Bordeaux on the outer edges of the petals. Fine light stripe rumning through the center of each petal. Lip speckled with ruby. Large flowers. Spikes long. A very odd variety and good strong grower.

$\begin{array}{lrccc} & \text { Bulbs } & \text { One-half Size } & \text { One-quarter Size } & \text { Bulblets } \\ \text { Per Bulb } & .50 & .25 & . \dot{5} & . . \dot{5} \\ \text { Per Doz. } & 5.00 & 2.50 & 1.25 & 5.00 \\ \text { Per } 100 & 40.00 & 20.00 & 10.00 & 5.00\end{array}$

No. 226-"PASADENA"

Turkish red iridescent, flame orange striped. Lip white overlaid with ruby. Blooms of enormous size, and petals flaring. Very tall spikes, each one a bouquet in itself.

\begin{tabular}{lcccc} 
Per Bulb & Bulbs & One-half Size & One-quarter Size & Bulblets \\
Per Doz. & 1.00 & .50 & .25 & .9 .25 \\
Per 100 & 80.00 & 400 & 2.50 & 1.25 \\
\hline
\end{tabular}

No. 232-"FLAME"

Flame orange. Yellow lip, well built spikes.

$\begin{array}{lcccc}\text { Per Bulb } & \text { Bulbs } & \text { One-half Size } & \text { One-quarter Size } & \text { Bulblets } \\ \text { Per Doz. } & 1.00 & .50 & .25 & . \ddot{5} \dot{5} \\ \text { Per 100 } & 10.00 & 5.00 & 2.50 & 1.20\end{array}$

No. 234 -BEI.VEDERE"

Grenadine pink, overlaid with strawberry pink. Lip, apricot with large spot of pomegranate purple, also a faint stripe of greenish yellow running through the center of the lip. Fine long spikes.

$\begin{array}{lr}\text { Per Bulb } & \text { Bulbs } \\ \text { Per Doz. } & 50.00 \\ \text { Per } 100 & 400.00\end{array}$

$\begin{array}{ccc}\text { One-half Size } & \text { One-quarter Size } & \text { Bulblets } \\ 2.50 & 1.25 & .75 \\ 25.00 & 12.50 & 6.25\end{array}$

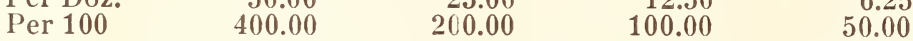

No. 236-"MARIN"

Picric yellow, with faint traces of ruby in the throat. Rufled edges, fine cut flower variety:

$\begin{array}{lrrrrr}\text { Per Bulb } & \text { Bulbs } & \text { One-half Size } & \text { One-quarter Size } & \text { Bulblets } \\ \text { Per Doz. } & .50 & .25 & .95 & .7 \dot{5} \\ \text { Per 100 } & 5.00 & 2.50 & 1.25 & 5.00\end{array}$

No. 237-“"THOMAS A. EDISON"

Clear Begonia Rose. Large deep blood red spots on lower petals. Flowers very large. First class show variety.

$\begin{array}{lcccc}\text { Bety. } & \text { Bulbs } & \text { One-half Size } & \text { One-quarter Size } & \text { Bulblets } \\ \text { Per Bulb } & 5.00 & 2.50 & 1.25 & .75 \\ \text { Per Doz. } & 50.00 & 25.00 & 12.50 & 6.25 \\ \text { Per 100 } & 400.00 & 260.00 & 100.00 & 50.00\end{array}$

No. 240_"SALOME JANE"

Geranium pink, lower petals scarlet spotted with deep ruby. Flowers very large. A beauty.

$\begin{array}{lcccc} & \text { Bulbs } & \text { One-half Size } & \text { One-quarter Size } & \text { Bulblets } \\ \text { Per Bulb } & 1.00 & .50 & .25 & 1.2 \dot{5} \\ \text { Per Doz. } & 10.00 & 5.00 & 2.50 & 1.20 \\ \text { Per 100 } & 80.00 & 40.00 & 20.00 & 10.00\end{array}$

No. 241-"VICTORIA FREDERICKS"

Eosine pink, overlaid with flame orange. Yellow lip with ruby speckles. Flowers immense Early flowering. One of the finest pinks. Stock limited, one bulb to each customer.

$\begin{array}{lcccc}\text { ering. One of the finest pinks. } & \begin{array}{c}\text { Stock limited, one bulb to each customer. } \\ \text { One-half Size }\end{array} \\ \text { One-quarter Size } & \text { Bulblets } \\ \text { Per Bulb } & 5.00 & 2.50 & 1.25 & .75 \\ \text { Per Doz } & \ldots . & \ldots & \ldots & \ldots \\ \text { Per } 100 & \ldots & \ldots & \ldots & \ldots\end{array}$

No. 244 -BOLINAS"

Cream white with faint ruby stripes. Lip, pale sulphur tinge. Fine, full, long and well built spikes. Flowers of good size.

$\begin{array}{lrccc} & \text { Bulbs } & \text { One-half Size } & \text { One-quarter Size } & \text { Bulblets } \\ \text { Per Bulb } & 2.00 & 1.00 & .50 & .00 \\ \text { Per Doz. } & 20.00 & 10.00 & 5.00 & 2.50 \\ \text { Per } 100 & 160.00 & 80.00 & 40.00 & 20.00\end{array}$

No. 246-"MIMI STAHLBAUM" Creamy white tinged with very pale orchid. Lip yellow with small ruby spots. An exquisite
variety.

Per Bulb

Per 100
1.50
15.00

120.00

.75
7.50

7.50
60.00

.50
3.75$$
\begin{array}{r}
30.00 \\
240.00
\end{array}
$$

240.00

\begin{abstract}
120.00
\end{abstract}




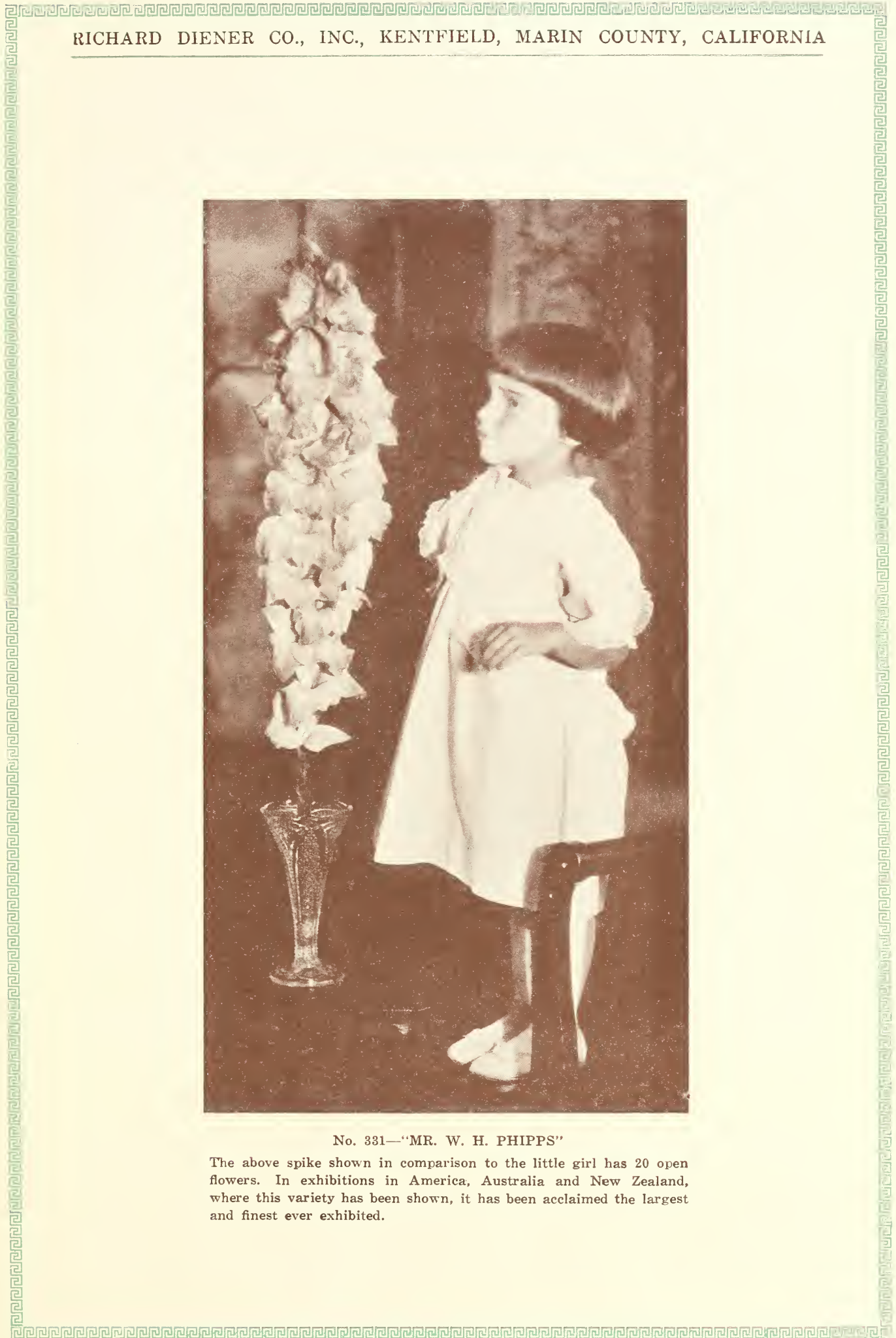




\section{No. 257- "CALIFORNIA GIANT"}

Light mallow purple, thin white stripe running through center of each petal. Very large.

$\begin{array}{lcccc} & \text { Bulbs } & \text { One-half Size } & \text { One-quarter Size } & \text { Bulblet } \\ \text { Per Bulb } & 3.00 & 1.50 & .75 & .50 \\ \text { Per Doz. } & 30.00 & 15.00 & 7.50 & 3.75 \\ \text { Per 100 } & 240.00 & 120.00 & 60.00 & 30.00\end{array}$

\section{No. 265- "ANNE THOMPSON"}

Very pale sulphur yellow with a faint trace of shell pink and splashes of ruby in the throat. A

first class variety.

$\begin{array}{lrccc}\text { variety. } & \text { Bulbs } & \text { One-half Size } & \text { One-quarter Size } & \text { Bulblets } \\ \text { Per Bulb } & 5.00 & 2.50 & 1.25 & .75 \\ \text { Per Doz. } & 50.00 & 25.00 & 12.50 & 6.25 \\ \text { Per } 100 & 400.00 & 200.00 & 100.00 & 50.00\end{array}$

\section{No. 266-"CAROLINE ESBERG"}

Flame scarlet red. No markings with the exception of a fine crimson stripe through the center of the lower petals. Most vivid red imaginable. The color of this variety does not fade under any condition.

$\begin{array}{lrccr}\text { Per Bulb } & \text { Bulbs } & \text { One-half Size } & \text { One-quarter Size } & \text { Bulblets } \\ \text { Per Doz. } & 4.00 & 2.00 & 1.00 & .60 \\ \text { Per 100 } & 40.00 & 20.00 & 10.00 & 5.00 \\ & 320.00 & 160.00 & 80.00 & 40.00\end{array}$

No. 268_"PAPILIO"

Shrimp pink and apricot. Lip real lemon yellow, speckled with ruby. Immense flowers, very long, strong spikes. Lip real 10

$\begin{array}{lrccc}\text { ng spikes. } & \text { Bulbs } & \text { One-half Size } & \text { One-quarter Size } & \text { Bulblets } \\ \text { Per Bulb } & 5.00 & 2.50 & 1.25 & .75 \\ \text { Per Doz. } & 50.00 & 25.00 & 12.50 & 6.25 \\ \text { Per 100 } & 400.00 & 200.00 & 100.00 & 50.00\end{array}$

No. 269_"GEISHA"

Cream yellow, bright canary center, overlaid with ruby. Long sturdy spikes.

$\begin{array}{lrccr} & \text { Bulbs } & \text { One-half Size } & \text { One-quarter Size } & \text { Bulblets } \\ \text { Per Bulb } & 10.00 & 5.00 & 2.50 & 1.25 \\ \text { Per Doz. } & 100.00 & 50.00 & 25.00 & 12.50 \\ \text { Per 100 } & 800.00 & 400.00 & 200.00 & 100.00\end{array}$

No. 276-"BERNARD SHAW"

Very pale lilac delicately penciled with light phlox purple. Faint dash of lemon on lower petals. Flowers immense, very open Amaryllis shaped. Lollg well built spikes.

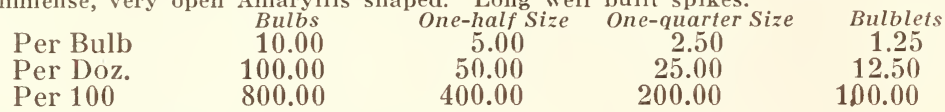

No. 277- "BEATRIZ MICHELENA"

Peach red overlaid with fiery orange. A thin ruby stripe through lower petals surrounded with white speckles. Large flowers, with a large amount of flowers open at one time, same of very lasting quality. This variety is perfect in every detail and a masterpiece in Gladioli.

$\begin{array}{lcccc} & \text { Bulbs } & \text { One-half Size } & \text { One-quarter Size } & \text { Bulblets } \\ \text { Per Bulb } & 4.00 & 2.00 & 1.00 & .60 \\ \text { Per Doz. } & 40.00 & 20.00 & 10.00 & 5.00 \\ \text { Per } 100 & 320.00 & 160.00 & 80.00 & 40.00\end{array}$

No. 281- "DEL MONTE"

Aster purple, lighter toward the center. White stripe through the center of each petal. White lip striped with ruby. Large flowers on strong well built spikes.

$\begin{array}{lcccc} & \text { Bulbs } & \text { One-half Size } & \text { One-quarter Size } & \text { Bulblets } \\ \text { Per Bulb } & 5.00 & 2.50 & 1.25 & .75 \\ \text { Per Doz. } & 50.00 & 25.00 & 12.50 & 6.25 \\ \text { Per 100 } & 400.00 & 200.00 & 100.00 & 50.00\end{array}$

No. 282_" "PHINI"

Cameo pink blotched and striped with aster purple. Large spots of purple on the lip. Very free flowering. Enormous blooms. Fine long spikes. An improvement on AMERICA.

$\begin{array}{lrrrr} & \text { Bulbs } & \text { One-half Size } & \text { One-quarter Size } & \text { Bulblets, } \\ \text { Per Bulb } & 10.00 & 5.00 & 2.50 & 1.00 \\ \text { Per Doz } & 100.00 & 50.00 & 25.00 & 10.00 \\ \text { Per } 100 & \ldots & \ldots & \ldots & \ldots\end{array}$

No. 283 "NECTARINE"

Peach red, with yellow and ruby centers. Flowers large. Long spikes. Very heavy propagator.

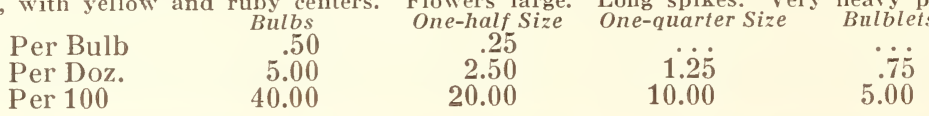




\section{RICHARD DIENER CO., INC., KENTFIELD, MARIN COUNTY, CALIFORNIA}

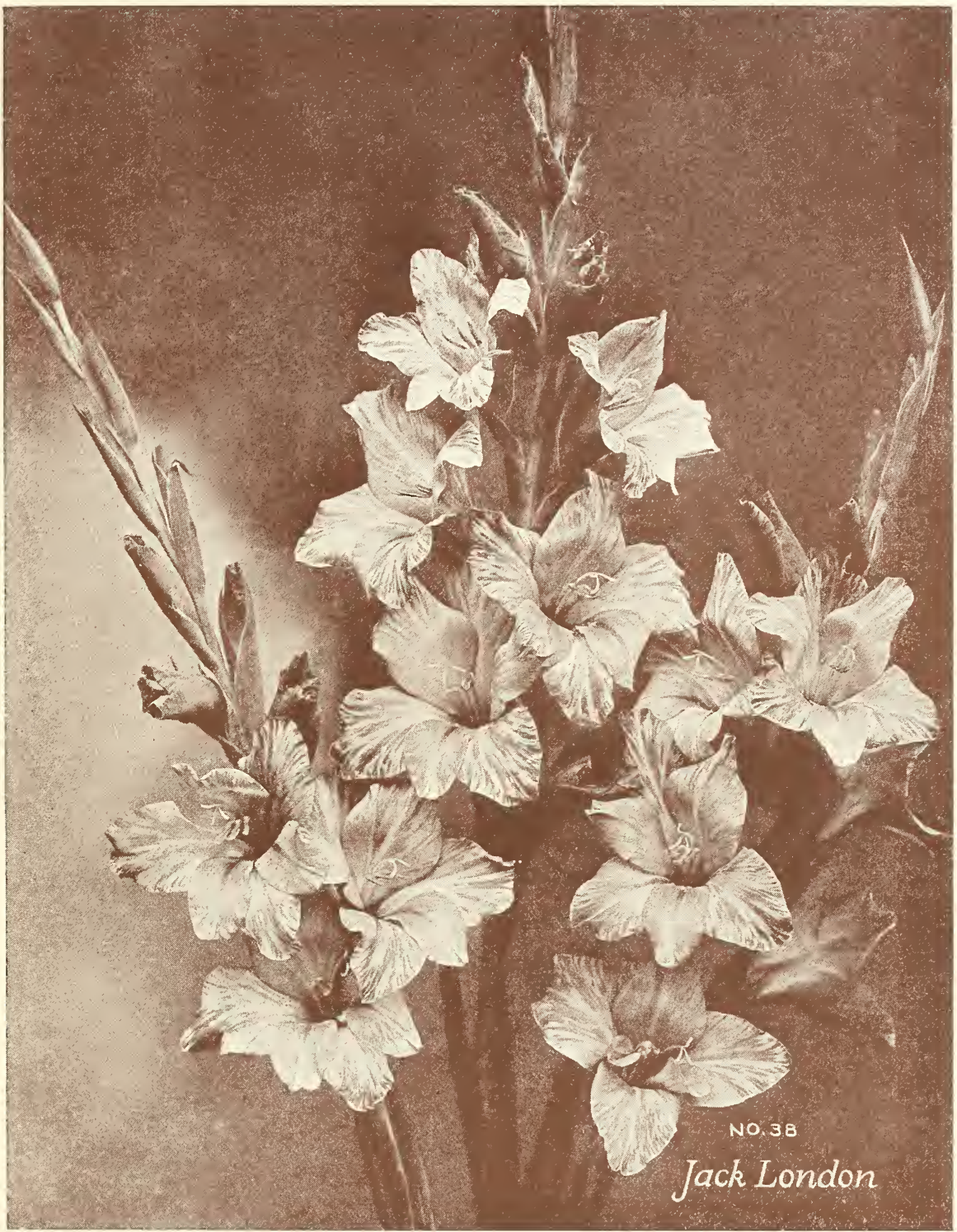

THE ABOVE THREE SPIKES WERE DERIVED FROM ONE SINGLE INCH AND A HALF BULB

Mr. C. E. Oldacre of Toronto, Canada, writes us the following on September 20, 1920:

"Meritorious articles deserve favorable notice and commendation, and that applies to your Gladioli. Of some forty varieties, most of which are considered the cream of the Gladioli world, which I had this year, your No. 38 Jack London is right up in the front ranks with all points considered. Neither your short catalogue description nor your colored illustration of same does this flower justice.

"It is of great substance, a most beautiful color or color combination, and the individual flowers are well placed, forming a shapely, erect spike, which carries five to seven wide open flowers at a time that makes a bouquet in itself. More than this, it seems to be a most lasting variety, as the individual fiowers retain their true color for twice as long a period as do many others. No. 38 is easily the top-notcher in its color class, and should be one of any first-class selection of varieties.

"Wren friends were looking over my Glads the question was asked as to what six varieties were the most admired, and No. 38 was always one of the six that were chosen." 


\section{No. 285-"MRS. RUDOLPH SPRECKELS"}

Light shrimp pink with bright rose stripes. Lip has apricot shadings and a large spot of ruby. Long full spikes. A-1 show and cut flower variety,

\begin{tabular}{|c|c|c|c|c|}
\hline Per Bulb & $\begin{array}{r}\text { Bulbs } \\
.75\end{array}$ & $\begin{array}{c}\text { One-half Size } \\
.40\end{array}$ & One-quarter Size & Bulblets \\
\hline Per Doz. & 7.50 & 3.75 & 2.00 & 1.00 \\
\hline Per 100 & 60.00 & 30.00 & 15.00 & 7.50 \\
\hline
\end{tabular}

No. 286- "RUTH TAYLOR"

Snow white, without any markings. Violet stamens. Flowers 6 to 7 inches in diameter. Very long strong spikes. We believe that this is the first real white of large size.

$\begin{array}{lrccc}\text { Per Bulb } & \text { Bulbs } & \text { One-half Size } & \text { One-quarter Size } & \text { Bulblets } \\ \text { Per Doz. } & 15.00 & 7.50 & 3.75 & 2.00 \\ \text { Per } 100 & 150.00 & 75.00 & 37.50 & 18.75 \\ & \ldots & \ldots . & \ldots & \ldots\end{array}$

\section{No. 293-"POLA NEGRI"}

Apricot with yellow lip. Ruffled, shading into rose and salmon on outer edges. One of the lovliest color combinations ever seen. The flowers on the spikes sometimes come irregular. It is a very heavy propagator. We especially recommend this variety, as all who have seen it have placed orders for it.

$\begin{array}{lcccc} & \text { Bulbs } & \text { One-half Size } & \text { One-quarter Size } & \text { Bulblets } \\ \text { Per Bulb } & 1.00 & .50 & .25 & . \dot{5} \\ \text { Per Doz. } & 10.00 & 5.00 & 2.50 & 1.25 \\ \text { Per 100 } & 80.00 & 40.00 & 20.00 & 10.00\end{array}$

\section{No.295- "BLASCO IBANEZ"}

Very deep Dahlia Purple with a lighter stripe through the upper petals. Very large size. An extra fine variety.

$\begin{array}{lcccc} & \text { Bulbs } & \text { One-half Size } & \text { One-quarter Size } & \text { Bulblets } \\ \text { Per Bulb } & 10.00 & 5.00 & 2.50 & 1.25 \\ \text { Per Doz. } & 100.00 & 50.00 & 25.00 & 12.50 \\ \text { Per } 100 & 800.00 & 400.00 & 200.00 & 100.00\end{array}$

\section{No. 297-"MRS. ROBERT CUNNINGHAM"}

Cameo pink overlaid with lilac and ruby. Flowers of the very largest size. Many blossoms open at one time. One of the largest Gladioli yet produced.

$\begin{array}{lcccc} & \begin{array}{c}\text { Bulbs } \\ \text { Per Bulb }\end{array} & 5.00 & \begin{array}{c}\text { One-half Size } \\ \text { One-quarter Size }\end{array} & \text { Bulblets } \\ \text { Per Doz. } & 50.00 & 2.50 & 1.25 & .75 \\ \text { Per 100 } & \ldots . & 25.00 & 12.50 & 6.25 \\ & & \ldots & \ldots & \ldots\end{array}$

No. 298_"SONOMA'

Cream yellow. Lip finely striped with crimson. Fine, long, well built spikes.

$\begin{array}{lcccc} & \text { Bulbs } & \text { One-half Size } & \text { One-quarter Size } & \text { Bulblets } \\ \text { Per Bulb } & 1.00 & .50 & .25 & .25 \\ \text { Per Doz. } & 10.00 & 5.00 & 2.50 & 1.25 \\ \text { Per 100 } & 80.00 & 40.00 & 20.00 & 10.00\end{array}$

No. 304 "SAN JOSE"

Center lemon yellow shading into apricot on outside petals. Peach red striping. Flowers large. Very fine well built spikes.

$\begin{array}{lrccc} & \text { Bulbs } & \text { One-half Size } & \text { One-quarter Size } & \text { Bulblets } \\ \text { Per Bulb } & 5.00 & 2.50 & 1.25 & .75 \\ \text { Per Doz. } & 50.00 & 25.00 & 12.50 & 6.25 \\ \text { Per } 100 & 400.00 & 200.00 & 100.00 & 50.00\end{array}$

No. 305- "J. A. CARBONE"

Orange salmon, very iridescent, darker shadings toward the outer edges of petals. Yellow center. Fine, long, well built spikes. One of our largest and finest productions.

$\begin{array}{lrccc} & \text { Bulbs } & \text { One-half Size } & \text { One-quarter Size } & \text { Bulblets } \\ \text { Per Bulb } & 30.00 & 15.00 & 7.50 & 3.75 \\ \text { Per Doz. } & 300.00 & 150.00 & 75.00 & 37.50 \\ \text { Per } 100 & 2400.00 & 1200.00 & 600.00 & 300.00\end{array}$

No. 311 " "GLADIS PLATH"

Mallow purple, darker near the outer edges of the petals. Large crimson spots on the lip. White stripe through each petal. Orchid flowering. Beautiful variety.

$\begin{array}{lrrrr} & \text { Bulbs } & \text { One-half Size } & \text { One-quarter Size } & \text { Bulblets } \\ \text { Per Bulb } & .75 & .40 & 0.00 & .00 \\ \text { Per Doz. } & 7.50 & 3.75 & 2.00 & 1.00 \\ \text { Per 100 } & 60.00 & 30.00 & 15.00 & 7.50\end{array}$




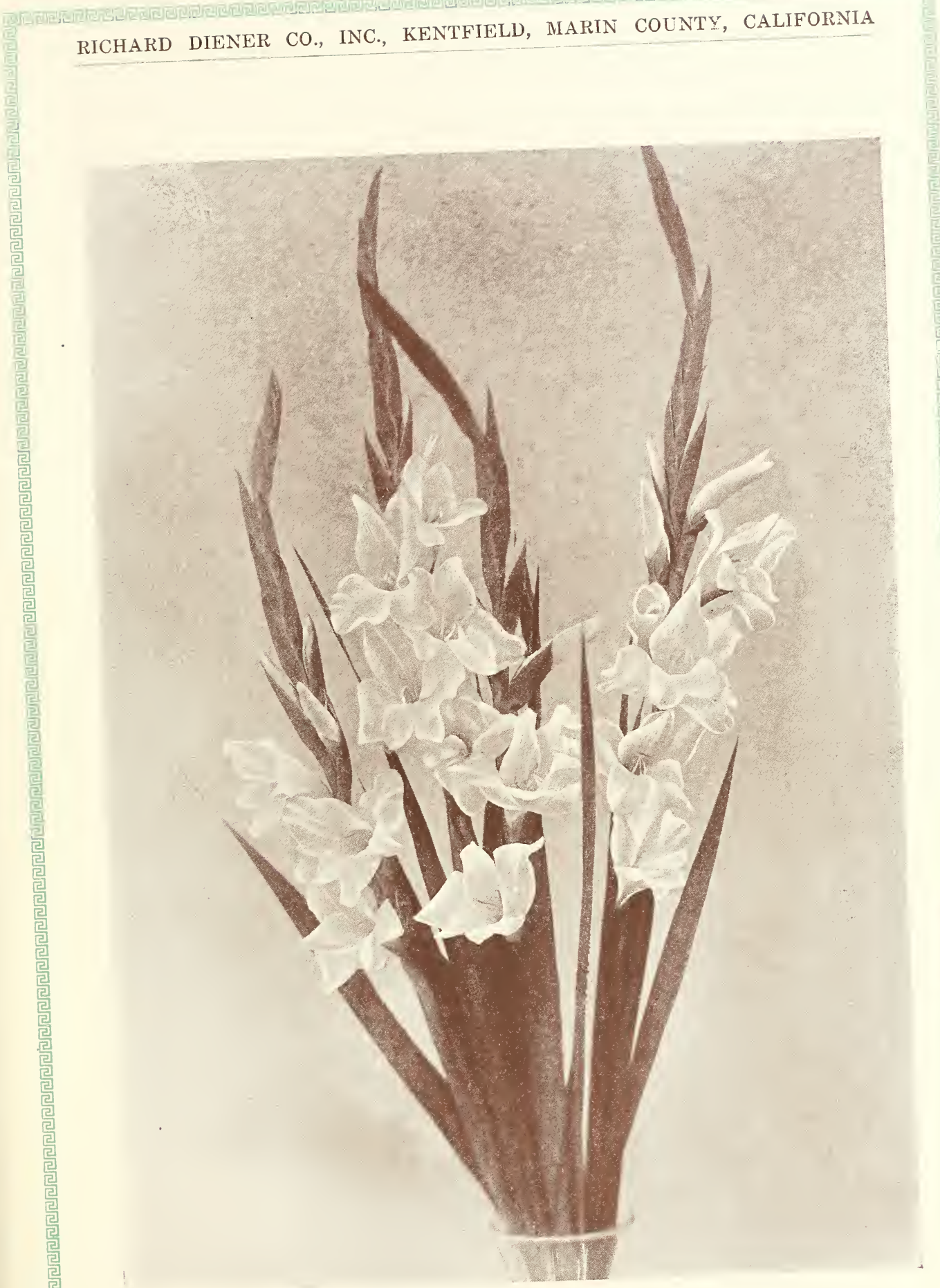

No. 286-"RUTH TAYLOR" 


\section{No. 312-"MRS. RICHARD LOHRMAN"}

Creamy shell pink with pale yellow lip. A coloring rivaling the finest orchid. Flowers are very large and edges heavily rumed. Long, sturdy spikes which sometimes kink, wonderful for decorative purposes. One of the greatest productions in Gladioli and of indescribable beauty.

$\begin{array}{lcccc} & \text { Bulbs } & \text { One-half Size } & \text { One-quarter Size } & \text { Bulblets } \\ \text { Per Bulb } & 1.50 & 7.75 & .40 & .00 \\ \text { Per Doz. } & 15.00 & 7.50 & 3.75 & 2.00 \\ \text { Per 100 } & 120.00 & 60.00 & 30.00 & 15.00\end{array}$

\section{No. 315- "ELIZABETH HARDEE"}

Watermelon pink, with Nopal red stripes and blotches. Ruby lip with cream white stripe appearing in the center, surrounded by cream and red speckles. A beautiful and attractive variety.

$\begin{array}{lrrrr} & \text { Bulbs } & \text { One-half Size } & \text { One-quarter Size } & \text { Bulblets } \\ \text { Per Bulb } & 3.00 & 1.50 & .75 & .50 \\ \text { Per Doz. } & 30.00 & 15.00 & 7.50 & 3.75 \\ \text { Per 100 } & 240.00 & 120.00 & 60.00 & 30.00\end{array}$

No. 317—"LUCIA"

Amaranth pink with rose stripings. Flowers of the very largest size.

$\begin{array}{lrccc} & \text { Bulbs } & \text { One-half Size } & \text { One-quarter Size } & \text { Bulblets } \\ \text { Per Bulb } & 5.00 & 2.50 & 1.25 & .75 \\ \text { Per Doz. } & 50.00 & 25.00 & 12.50 & 6.25 \\ \text { Per 100 } & 400.00 & 200.00 & 100.00 & 50.00\end{array}$

No. 318- "KATHARINE F. HESS"

Geranium pink showing shadings of flame, lighter toward the center. Very large flowers and long spikes. Fine grower. A beautiful variety with a great future.

$\begin{array}{lcccc} & \text { Bulbs } & \text { One-half Size } & \text { One-quarter Size } & \text { Bulblets } \\ \text { Per Bulb } & 1.00 & .50 & .25 & .2 \dot{2} \\ \text { Per Doz. } & 10.00 & 5.00 & 2.50 & 1.25 \\ \text { Per } 100 & 80.00 & 40.00 & 20.00 & 10.00\end{array}$

No. 320-"ESTHER OLSON"

Seashell pink, shading to shrimp pink on the outer edges. Faint ruby stripes rumning through the lower petals. Lip straw color. Exquisite color and perfect in every way.

$\begin{array}{lcccc} & \text { Bulbs } & \text { One-half Size } & \text { One-quarter Size } & \text { Bulblets } \\ \text { Per Bulb } & 3.00 & 1.50 & .75 & .50 \\ \text { Per Doz. } & 30.00 & 15.00 & 7.50 & 3.75 \\ \text { Per 100 } & 240.00 & 120.00 & 60.00 & 30.00\end{array}$

No. 321_"ELF"

Lemon yellow when in bud. Open flowers pure white, lip delicate lemon. Large flowers on a perfectly built spike. Even the smallest bulblet will produce a fine flower spike. A commercial variety of great promise.

$\begin{array}{lrccc} & \text { Bulbs } & \text { One-half Size } & \text { One-quarter Size } & \text { Bulblets } \\ \text { Per Bulb } & .50 & .25 & 1 . \dot{2} & . \dot{5} \dot{5} \\ \text { Per Doz. } & 5.00 & 2.50 & 10.00 & 5.00 \\ \text { Per } 100 & 40.00 & 20.00 & 10.00\end{array}$

No. 324 -"W. McGAVIN"

Very light rosalane purple with rose blotches. White center. Very beautiful color. Spikes very long and well built.

$\begin{array}{lcccc}\text { well built. } & \text { Bulbs } & \text { One-half Size } & \text { One-quarter Size } & \text { Bulblets } \\ \text { Per Bulb } & 10.00 & 5.00 & 2.50 & 1.25 \\ \text { Per Doz. } & 100.00 & 50.00 & 25.00 & 12.50 \\ \text { Per 100 } & \ldots & \ldots & \ldots . & \ldots\end{array}$

No. 326- "PHYLOS"

Silvery rosalane pink. Cerise and silver overlaid. Flowers large and spikes very long. Odd and attractive.

$\begin{array}{lcccc} & \text { Bulbs } & \text { One-half Size } & \text { One-quarter Size } & \text { Bulblets } \\ \text { Per Bulb } & 1.00 & .50 & .25 & . \dot{2} \dot{5} \\ \text { Per Doz. } & 10.00 & 5.00 & 2.50 & 10.00 \\ \text { Per 100 } & 80.00 & 40.00 & 20.00 & 100\end{array}$

No. 327-"DR. ADOLPH ROSENTHAL"

Real geranium pink, overlaid with scarlet. White stripes through lower petals. Flowers large on fine, well built spikes. . An exquisite variety.

\begin{tabular}{|c|c|c|c|c|}
\hline iull & Bulbs & One-half Size & One-quarter Size & Bulblets \\
\hline $\begin{array}{l}\text { Per Bulb } \\
\text { Per Doz. }\end{array}$ & $\begin{array}{r}1.00 \\
10.00\end{array}$ & 5.00 & 2.250 & 125 \\
\hline Per 100 & 80.00 & 40.00 & 20.00 & 8.00 \\
\hline
\end{tabular}




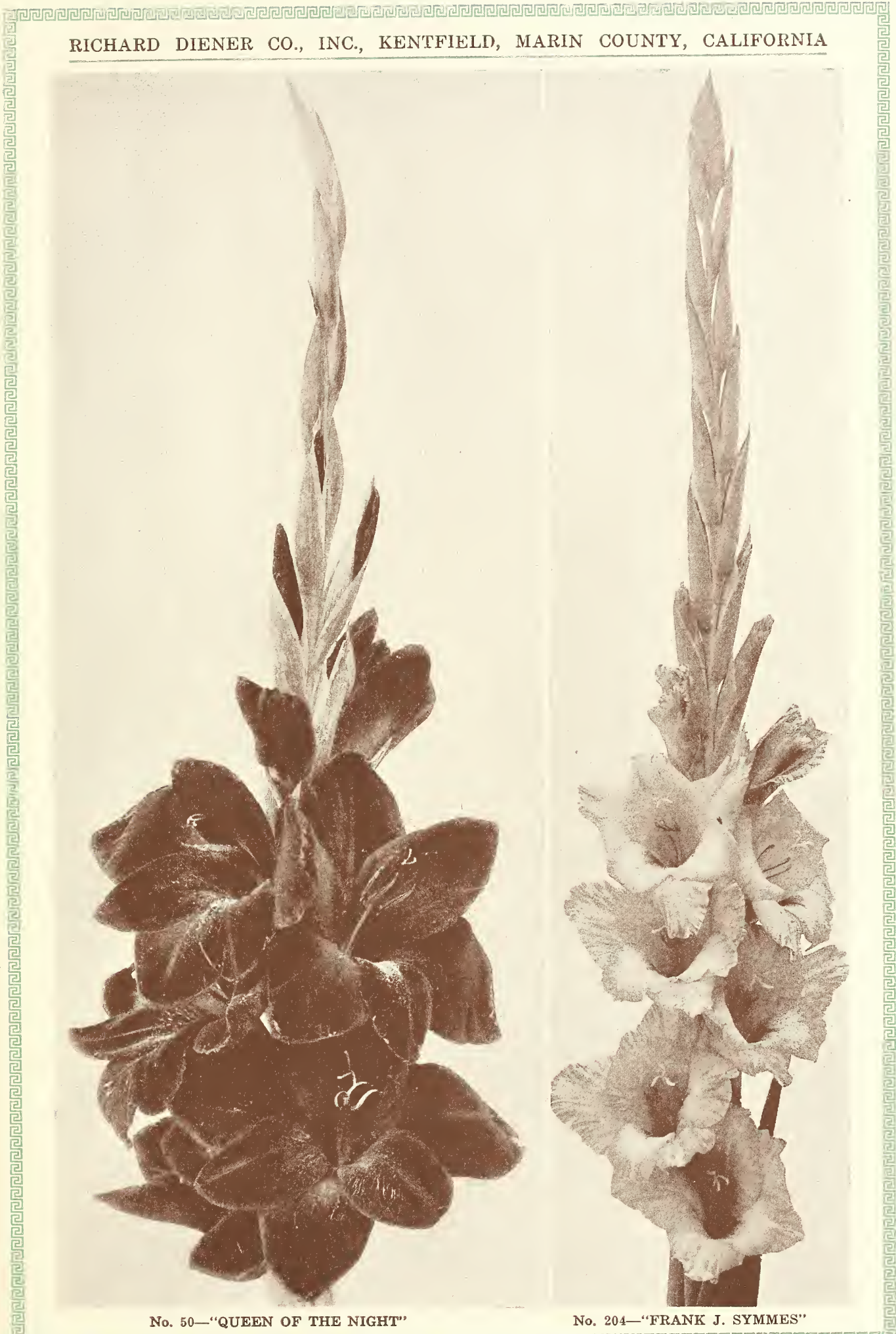

No. 50-"QUEEN OF THE NIGHT"

No. 204-"FRANK J. SYMMES"

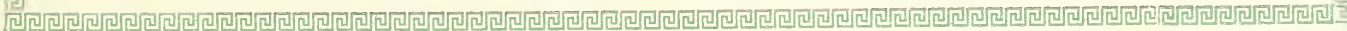




\section{No. 328---"BUBBLES"}

Flesh color overlaid with shrimp pink. Lower petals of light greenish yellow. One spike forms a large close set bouquet. A beauty. Should be in every collection.

$\begin{array}{lrrrr}\text { Per Bulb } & \begin{array}{r}\text { Bulbs } \\ 2.00\end{array} & \begin{array}{c}\text { One-half Size } \\ \text { One-quarter Size }\end{array} & \text { Bulblets } \\ \text { Per Doz. } & 20.00 & 1.00 & .50 & . \dot{0} \\ \text { Per 100 } & 160.00 & 80.00 & 4.00 & 2.50 \\ & & & 40.00 & 20.00\end{array}$

No. 329-"MOONBEAM"

Light chamois color. Canary center. Of fine clean growth.

\begin{tabular}{|c|c|c|c|}
\hline Per & $\begin{array}{l}\text { Bulbs } \\
10.00\end{array}$ & $\begin{array}{c}\text { One-half Size } \\
5.00\end{array}$ & One-quarter Size \\
\hline & 100.0 & 50.00 & 25.00 \\
\hline & 800.00 & 400.00 & 200.00 \\
\hline
\end{tabular}

No. 330-"LORELEY"

True canary yellow overlaid with lilac. Ruby throat. Cican grower and heavy propagator. One of the best yellows ever produced.

$\begin{array}{lrccc}\text { yellows ever produced. } & \text { Bubs } & \text { One-half Size } & \text { One-quarter Size } & \text { Bulblets } \\ \text { Per Bulb } & .25 & 1.25 & .7 .5 & . .45 \\ \text { Per Doz. } & 2.50 & 10.00 & 5.00 & 2.50 \\ \text { Per 100 } & 20.00 & 10.00 & .5\end{array}$

\section{No. 331 - "NR. W. H. PHIPPS"}

La France pink overlaid with light rose salmon, lighter toward the center. Lower petals very La France pink overlaid with light rose salmon, lighter toward the center. Lower petals very
faintly striped and speckled with ruby. Flowers enormous. A masterpiece in Gladioli, the great-
est of our productions.

$\begin{array}{lcccc}\text { productions. } & \text { Bulbs } & \text { One-half Size } & \text { One-quarter Size } & \text { Bulblets } \\ \text { Per Bulb } & 30.00 & 15.00 & 7.50 & 3.75 \\ \text { Per Doz. } & 300.00 & 150.00 & 75.00 & 37.50 \\ \text { Per 100 } & 2400.00 & 1200.00 & 600.00 & 300.00\end{array}$

No. 332- "ROSE ASH"

Corinthian red shading into Ashes of Roses color on the outer edges. Lower petals light yellow speckled with ruby. Flowers large, well set on the spike. This variety has a great future. "Rose Ash" was originated by Richard Diener, and all other claims of origination are false.

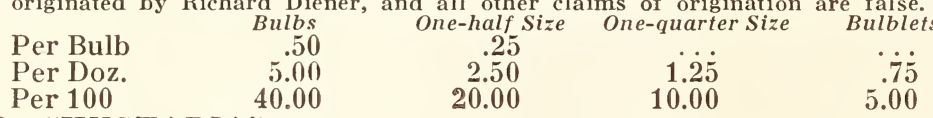

No. 333-"WISTARIA"

Orchid coloring, ruby and mauve striped. Ruby centers. Long spikes with flowers well placed.

Flowers large. Bulbs

\begin{tabular}{lrrrr} 
large. & Bulbs & $\begin{array}{c}\text { One-half Size } \\
\text { Per Bulb }\end{array}$ & $\begin{array}{c}\text { One-quarter Size } \\
\text { One- }\end{array}$ & $\begin{array}{c}\text { Bulblets } \\
\text { Pel Doz. }\end{array}$ \\
Per 100 & 2.50 & 1.25 & .65 & .35 \\
\hline
\end{tabular}

No. 331 -"EUDORA"

Rhodamine purple, white splashes in the center. Flowers large. Exceptionally fine growth.

$\begin{array}{lcccc}\text { e purple, white } & \begin{array}{c}\text { splashes in } \\ \text { Bulbs }\end{array} & \text { the center. Fowe-half Size } & \text { One-quarter Size } & \text { Bulblets } \\ \text { Oner } & 7.50 & 3.75 & 2.00 \\ \text { Per Bulb } & 15.00 & 75.00 & 37.50 & 18.75 \\ \text { Per Doz. } & 150.00 & 600 & 300.00 & 150.00\end{array}$

No. 335-"MR. HENRY BECHMAN"

lridescent steel blue, sulphur ycllow lip. Ligliter sulphur stripe through the center of each petal. Each petal has a pink tip. Something extraordinary.

$\begin{array}{lcccc} & \text { Bulbs } & \text { One-lalf Size } & \text { One-quarter Size } & \text { Bulblets } \\ \text { Per Bulb } & 5.00 & 2.50 & 1.25 & .75 \\ \text { Per Doz. } & 50.00 & 25.00 & 12.50 & 6.25 \\ \text { Per 100 } & 400.00 & 200.00 & 100.00 & 50.00\end{array}$

No. 336 -DR. F. E. BENNETT" Deep peach red overlaid with flame scarlet. Lip speckled with ruby and white. Most fiery red
imaginable. Flowers of the very largest size with a great many opening at one time. Exceptionally well placed on the stem. Most perfect red we have ever seen. This has been named in honor of the President of American Gladiolus Socieiv.

$\begin{array}{lcccc} & & \\ \text { Per Bulb } & \text { Bulhs } & \text { One-half Size } & \text { One-quarter Size } & \text { Bulblets } \\ \text { Per Doz. } & 300.00 & 15.00 & 7.50 & 3.75 \\ \text { Per Doz. } & 3000.00 & 1500.00 & 75.00 & 37.50 \\ \text { Per 100 } & 2400.00 & 1200.00 & 600.00 & 300.00\end{array}$

No. 337-"VIRGINIAN"

Rose red with bright vermilion spots on the outer petals. Deep Crimson Lake stripes through the center of the lower petals. Fine, long, well built spikes.

$\begin{array}{lcccc} & \begin{array}{c}\text { Bulbs } \\ \text { Per Bulb }\end{array} & 4.00 & \begin{array}{c}\text { One-half Size } \\ \text { One-quarter Size }\end{array} & \text { Bulblets } \\ \text { Per Doz. } & 40.00 & 2.00 & 1.00 & .60 \\ \text { Per 100 } & 320.00 & 20.00 & 10.00 & 5.00 \\ & & 160.00 & 80.00 & 30.00\end{array}$


RICHARD DIENER CO., INC., KEN'TFIELD, MARIN COUNTY, CALIFORNIA
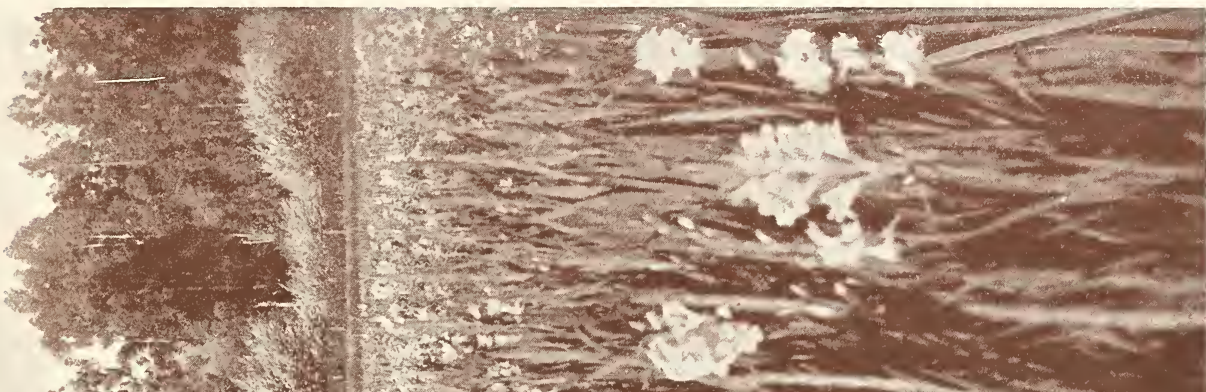

$723^{2}-3$
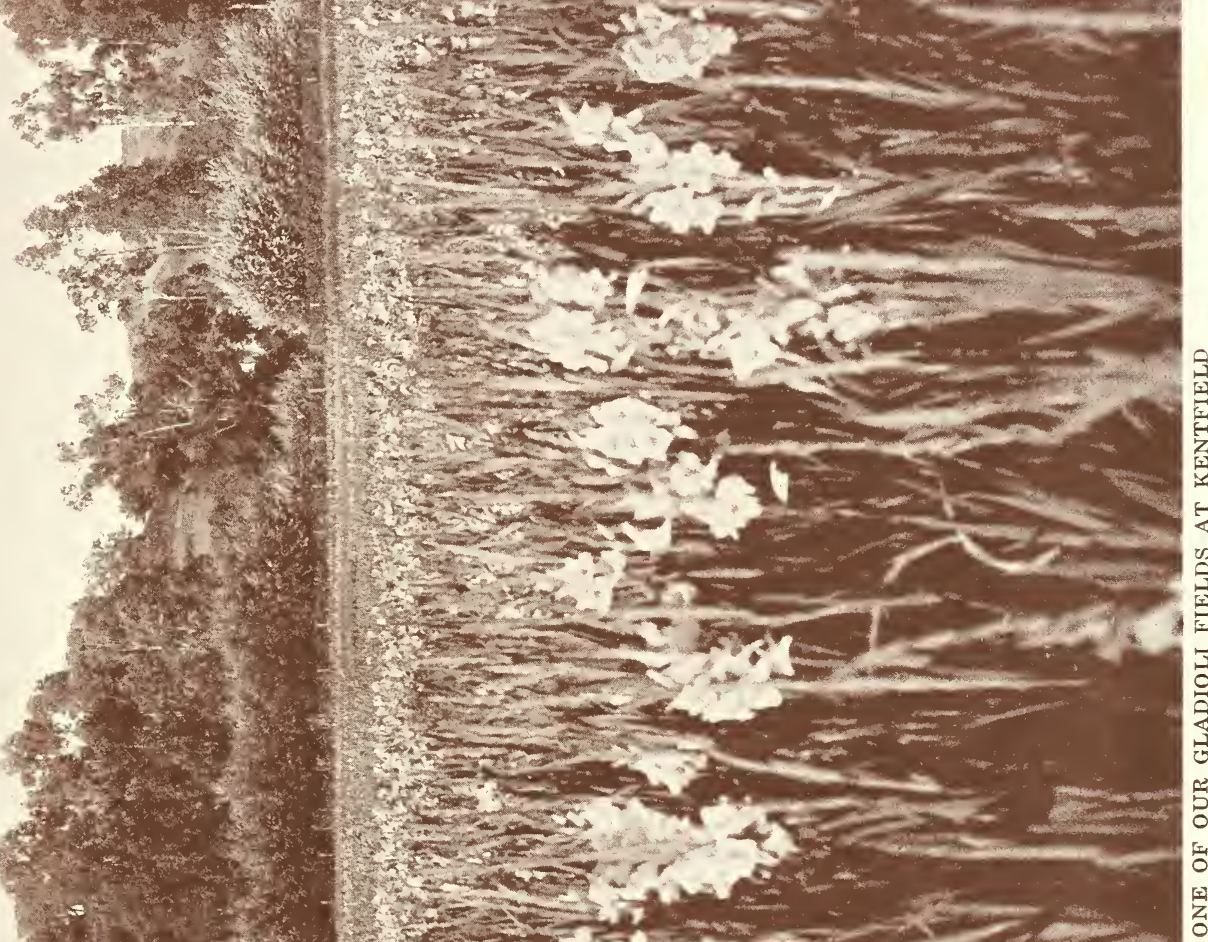

$x^{2}+2$
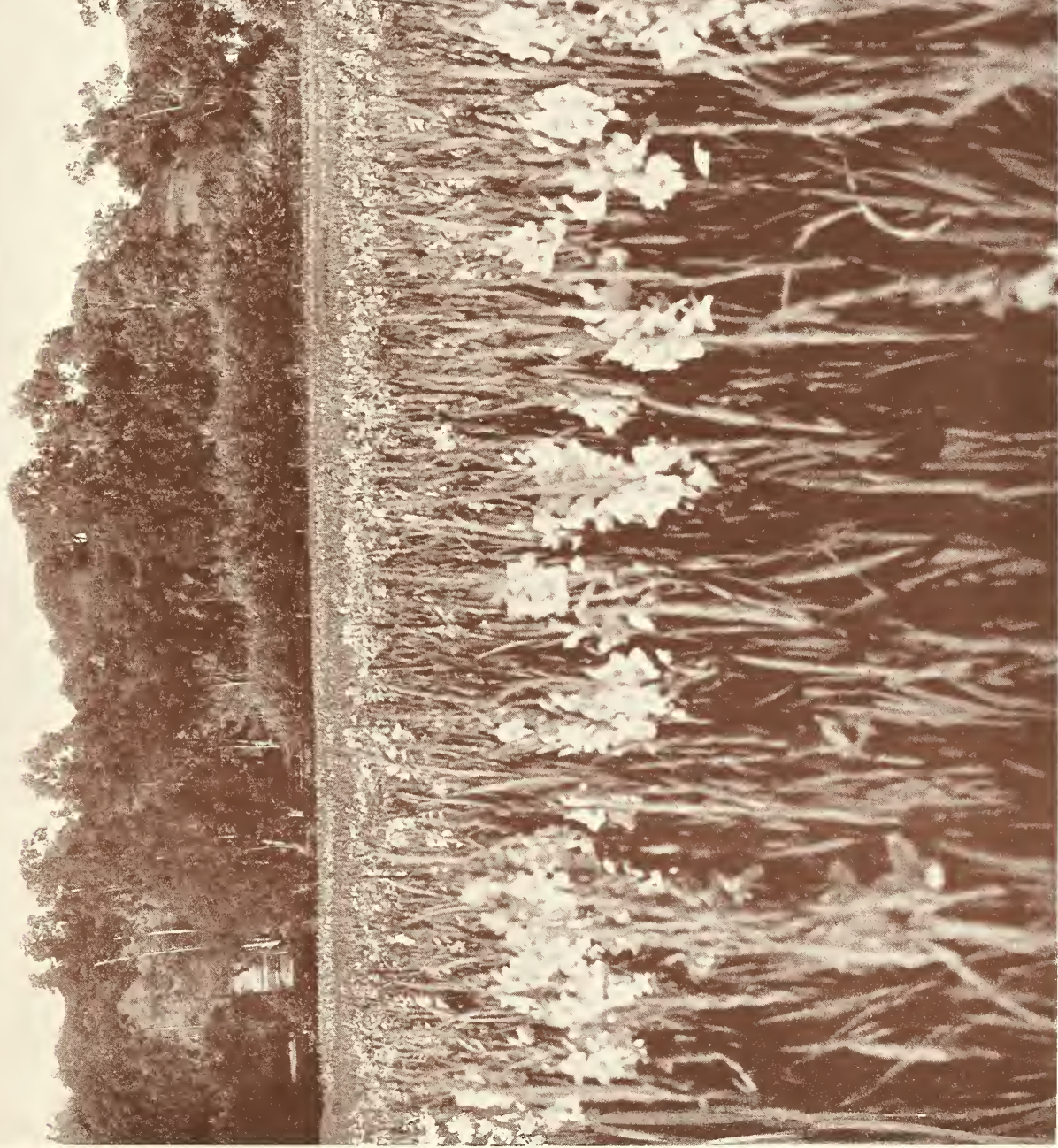


\section{No. 338-“GERALDINE FARRAR"}

Pale lavender-violet with deep violet spot on the lip. Unlike all the blue varieties, the growtl of this variety is very vigorous and strong. It has four side spikes on the main spike and brings two extra spikes from the bottom, out of the same eye, which is extremely new from any varieties ever seen. This variety has made a big impression with all of the visitors to this place, AIVARDED FIRST PRIZE for the finest blue variety at the convention of the American Gladiolus AVARDED FIRST PRIZE for the finest blue variety at the conv
Society, held at Rochester, N. Y., August 15 th and 16 th, 1923.

NOTE: As the demand for this new variety is so great, our stock is very limited.

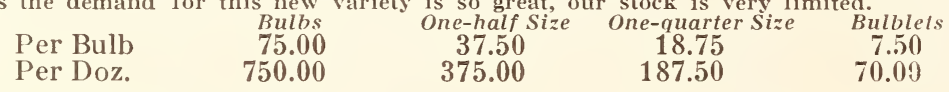

No. 339- "HENRY FORD"

Very dark Rlodamine purple shading into deeper towes near the center. Color very clear, like Anna Eberius, but much larger and cleaner. It is a tremendous propagator, and even the smallest bulblet will bring a flower spike three to four feet tall five months after planting. This variety far surpasses our very famous Anna Eberius, and we predict a great future for the "HENRY FORD." Bulbs one-half Size One-quarter Size Bulblets

$\begin{array}{lrrrr} & & & \\ \text { FORD." } & \text { Bulbs } & \text { One-half Size } & \text { One-quarter Size } & \text { Bulblets } \\ \text { Per Bulb } & 50.00 & 25.00 & 5.00 \\ \text { Per Doz. } & 500.00 & 250.00 & 12.50 & 50.00 \\ \text { Per 100 } & 4000.00 & 2000.00 & 1000.00 & 300.00\end{array}$

No. 341- "WAIKIKI"

Flesh color tinted with shrimp pink and showing faint touches of pale amaranth pink. Lip canary yellow. Deep throat with slight trace of ruby. Ruffled edges. Flowers very large.

$\begin{array}{lcccc} & \text { Bulbs } & \text { One-half Size } & \text { One-quarter Size } & \text { Bulblets } \\ \text { Per Bulb } & 1.00 & .50 & .25 & .2 .5 \\ \text { Per Doz. } & 10.00 & 5.00 & 2.50 & 1.25 \\ \text { Per } 100 & 80.00 & 40.00 & 20.00 & 10.00\end{array}$

No. 354 -THAIS VALDEMAR"

Iridescent old rose overlaid with bright vermilion. No markings. Large flowers. Very beautiful.

$\begin{array}{lcccc} & \text { Bulbs } & \text { One-half Size } & \text { One-quarter Size } & \text { Bulblets } \\ \text { Per Bulb } & 3.00 & 1.50 & .85 & .50 \\ \text { Per Doz. } & 30.00 & 15.00 & 7.50 & 3.75 \\ \text { Per } 100 & 240.00 & 120.00 & 60.00 & 30.00\end{array}$

No. 355- "LAGUNITAS"

Very pale shade between lilac and rose pink. Lip pale yellow overlaid with ruby. Long sturdy spikes.

$\begin{array}{lrccc} & \text { Bulbs } & \text { One-half Size } & \text { One-quarter Size } & \text { Bulblets } \\ \text { Per Bulb } & 5.00 & 2.50 & 1.25 & .75 \\ \text { Per Doz. } & 50.00 & 25.00 & 12.50 & 6.25 \\ \text { Per 100 } & 400.00 & 200.00 & 100.00 & 50.00\end{array}$

No. 358-"NOLENE"

Very deep shade between carmine and ox-blood, with darker velvet lip. Spike well built.

$\begin{array}{lcccc} & \text { Bulbs } & \text { One-half Size } & \text { One-quarter Size } & \text { Bulblets } \\ \text { Per Bulb } & 5.00 & 2.50 & 1.25 & .75 \\ \text { Per Doz. } & 50.00 & 25.00 & 12.50 & 6.25 \\ \text { Per 100 } & 400.00 & 200.00 & 100.00 & 50.00\end{array}$

No. 361- "CARMELITA"

Smoky pink, blotched and striped with steel blue. Yellow and slate speckled lip. Very large flowers. Very odd and beautiful.

$\begin{array}{lrccc}\text { Very odd and beautiful. } & \text { Bulbs } & \text { One-half Size } & \text { One-quarter Size } & \text { Bulblets } \\ \text { Per Bulb } & 3.00 & 1.50 & .85 & .50 \\ \text { Per Doz. } & 30.00 & 15.00 & 7.50 & 3.75 \\ \text { Per 100 } & 240.00 & 120.00 & 60.00 & 30.00\end{array}$

\section{No. 365-“TORADOR"}

Deep Nopal red with still darker seam around the edges. Very large funnel shaped flowers. Long well built spikes. Beautiful variety.

$\begin{array}{lrccc} & \text { Bulbs } & \text { One-half Size } & \text { One-quarter Size } & \text { Bulblets } \\ \text { Per Bulb } & 5.00 & 2.50 & 1.25 & .75 \\ \text { Per Doz. } & 50.00 & 25.00 & 12.50 & 6.25 \\ \text { Per } 100 & 400.00 & 200.00 & 100.00 & 50.00\end{array}$

No. 367- "CATTLAYA"

Amaranth pink, cream yellow center. Long fine spikes, exceptionally fine variety.

$\begin{array}{lrrrr} & \text { Bulbs } & \text { One-half Size } & \text { One-quarter Size } & \text { Bulblets } \\ \text { Per Bulb } & 3.00 & 1.50 & .85 & .50 \\ \text { Per Doz. } & 30.00 & 15.00 & 7.50 & 3.75 \\ \text { Per 100 } & 240.00 & 120.00 & 60.00 & 30.00\end{array}$




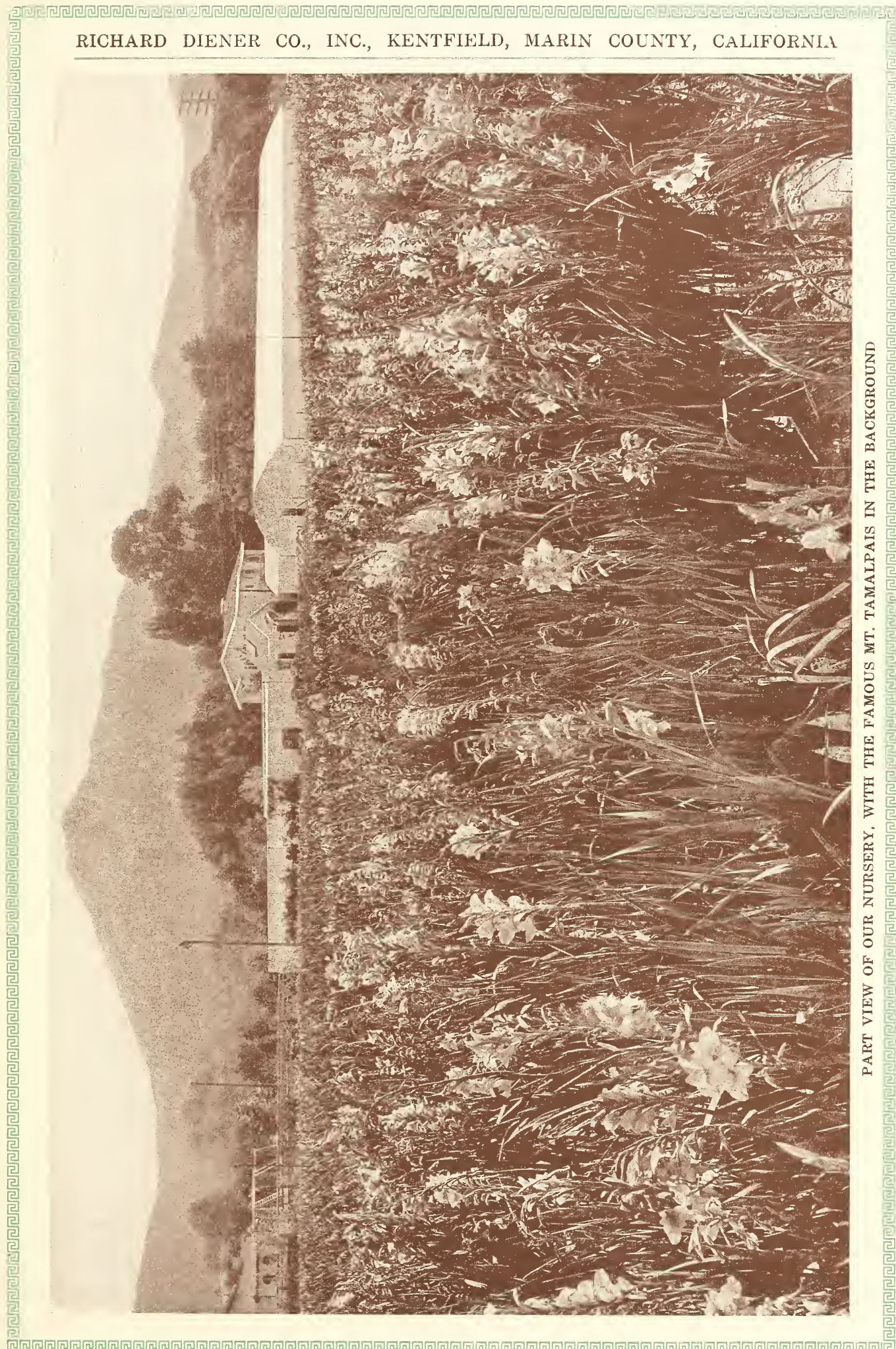




\section{Diener's Mixed Hybrids - 1923 Mixture}

Our old Hybrid Mixtures are entirely sold out, and the 1923 Mixture is made up of very fine stock, and unexcelled in size and coloring. The colors are mostly salmon, lilac, pink, yellow and orange-very few red. This is the very best mixture put on the market, and we are positive you will be highly satisfied with the same. It is made up of many thousands of unnamed varieties of our lyybrids. A wonderful combination of colors.

$\begin{array}{lcccc} & \text { Bulbs } & \text { One-half Size } & \text { One-quarter Size } & \text { Bulblets } \\ \text { Per Doz. } & 2.00 & 1.00 & .50 & \ldots \\ \text { Per } 100 & 15.00 & 7.50 & 3.75 & .0 . \\ \text { Per } 1000 & 75.00 & 37.50 & 18.75 & 5.00\end{array}$

We do not sell less than 1,000 bulblets. If you desire greater amounts of bulbs and bulblets than 1,000 , write us and we can make a more reasonable figure.

\section{Gladioli Seed}

Selected from all our named varieties and seedlings, well mixed. The sizes of the flowers range from 4 to 9 inches in diameter, and the height of the stems from 5 to 8 feet. The seedlings of this seed will flower in from 5 to 6 months after sowing if they receive proper care. It must be understood that the right size of the flowers and stems of seedling Gladiolus comes out in the second year of cultivation. These seedlings will range in all colors imaginable, but salmon, white, lilac, pink, rose-pink and yellow are the predominating colors. There is no other firm in the world that can put out any Gladiolus seed at such a low price, and of such high quality as these on the market.

Price of 10 grams (about 1,000 seeds) ..........\$ 1.00

Price per pound ............................ 35.00

\section{Method of Sowing Gladioli Seed}

The seed of Gladioli should be sown early in the spring. If the ground is fine, loose, sandy soil, then it is best right in the open ground, like onions in rows, The seeds should be covered as little as possible, and the soil used for covering should be finely sifted and contain half sand. Like all the seeds of plants of South African origin, Gladiolus seeds sprout unevenly, some sprout at once while others will not sprout until six to ten months after sowing under the same conditions. This is not due to the bad quality of the seed, but is a habit inherent in it where they grow wild. For this reason, seed beds should be made so that they should not otherwise be disturbed when the seedlings are lifted out, for all seeds will eventually come up. These beds should, in parts where it freezes, be covered with leaves thick enough so that the frost cannot reach the seeds in the beds. If sown out in boxes, and started indoors young plants of Gladiolus can be easily transplanted in the open if they are handled properly.

Gladiolus cormels or bulblets will grow faster if the outer hard shell is removed just before planting. They should not be covered more than one-half inch with soil; the ground should be enriched with old manure and worked over good and deep. If given the proper treatment, they will almost all be flowering five months after planting.

Richard Diener, Kentfield, Cal.

Dear Mr. Diener:- I have had some of the most wonderful flowers that I ever saw this year, and I want to say that they were, with a few exceptions some of your productions. Among the ones I had so many thrills over were, Queen of the Night, Mrs. Leon Douglas, Mrs. J. K. Armsby IVilliam Kent, Pola Negri, Ricliard Diener, J. A. Carbone and Mr. W. H. Phipps. Want to say that Phipps is certainly my ideal of a glad. The spike I have is over 16 inches long and has eight flowers open on it today, and from a $3 / 4$ inch bulb. What will it be next year when I will have a good sized No. 1? Pola Negri is very dainty, and I think it is a coming cut flower variety.

Had our County Fair here last week, and I exhibited over 50 varieties, and people came here from Dubuque, a city of 40,000 population, 28 miles away, to see them. Had more comments on the display, and I could not help notice how many people would point to William Kent and say what a pretty flower it was.

Thanking you for the wonderful stock you sent me, and hoping to have some more of your new productions next season. I am into the game now for nothing but the finest stock that will be able to get. My time is limited, and it will not cost me more to raise Richard Diener and such as it will America, Schwaben, King and such stock.

Awaiting your new list and hoping to hear from you in the near future, I beg to remain, Yours very truly,

JOSEPH A. FREYMANN. 


\section{Gladioli Collections}

We are offering this season, SPECIAL COLLECTIONS, made up of one bulb each of our most popular varieties, in order to give our customers the opportunity of getting a choice collection at a greatly reduced cost. However, in collections amounting to twenty-five dollars $(\$ 25.00)$ or over, we will give no discount, as we have allowed a greater reduction when offering the same. Kindly order by collection number.

SUBSTITUTION IN COLLECTIONS: We reserve the right to substitute when out of a variety listed in any of the collections. Please do not include varieties not mentioned in the same. If your order contains any such, we will be obliged to ignore it.

\section{WE PAY ALL POSTAGE}

\section{COLLECTION NO. 1}

1 Mrs. Wm. Kent......... .15

4 Sarah Lillie ........... $\mathbf{. 1 5}$

22 San Anselmo .......... $\quad .10$

31 Pres. C. C. Moore...... .15

32 Fairfax .............. $\quad .15$

38 Jack London .......... .25

162 Mary Roberts Rinehart.. $\quad .20$

164 Capt. A. C. Baker...... . .05

186 Lillian Webb ......... .05

189 Elizabeth Gerberding.... $\quad .10$

196 Helen Todd .......... $\quad .10$

198 Ross Valley........... .10

Value $\ldots \ldots \ldots \ldots \ldots \ldots \ldots \$ \overline{1.55}$

One each of above for......\$ 1.00

\section{COLLECTION NO. 2}

1 Mrs. Wm. Kent.... .... .. .15

19 Myrtle McNally ........ .25

39 Dr. Lincoln Cothran.... $\quad .25$

82 John Merle Coulter..... .25

90 Caroline Frear Burke.... .25

102 In a Collins . . . . . . . . . .50

130 Thos. T. Kent......... .25

145 Mrs. Cothran .......... $\quad .25$

187 Costa Rica ........... .25

198 Ross Valley .......... $\quad .10$

203 Sir Roger Casement..... . .25

204 Frank J. Symmes....... .25

205 David Starr Jordan. . . . . . .25

330 Loreley ............. .25

Value .............\$ $\overline{3.50}$

One each of above for......\$ 2.50
COLLECTION NO. 3

5 Aloha .............. .50

13 Sunset ........... .50

24 D. J. Whitney ........ 1.00

38 Jack London .......... .25

98 Mable Hubbard ........ .50

188 Mrs. Mary Stearns Burke. .50

191 Mrs. H. E. Bothin...... . .25

202 Mrs. John R. Walsh.... 1.00

204 Frank J. Symmes....... .25

221 London Smoke ........ .50

283 Nectarine ............ .50

311 Gladis Plath. .......... .75

330 Loreley ........... .25

Value ...............\$ 6.75

One each of above for.....\$\$ 5.00

\section{COLLECTION NO. 4}

23 American Beauty....... 1.00

101 Else Rose............ .75

107 Heart of Juanita....... .50

113 Mrs. Newell Vanderbilt. $\quad 1.00$

136 Adeline Kent ......... 1.00

191 Mrs. H. E. Bothin...... . .25

216 Martha Fernekes....... 1.00

218 Yosemite ........... 1.00

285 Mrs. Rudolph Spreckels. . .75

293 Pola Negri .......... 1.00

330 Loreley .............. . .25

332 Rose Ash............ .50

341 Waikiki ............ 1.00

Value .............\$ $\overline{10.00}$

One each of above for ......\$ 7.50 
COLLECTION NO. 5

\begin{tabular}{|c|c|c|}
\hline 19 & Myrtle McNally & .25 \\
\hline 23 & American Beauty & 1.00 \\
\hline 90 & Caroline Frear Burke.... & .25 \\
\hline 101 & Else Rose. & .75 \\
\hline 102 & In a Collins & .50 \\
\hline 113 & Mrs. Newell Vanderbilt. . & 1.00 \\
\hline 166 & Diener's White........ & 1.00 \\
\hline 173 & Hawaii. & .50 \\
\hline $1 / 6$ & Miss Maud Fay & .50 \\
\hline 30 & Mrs. Truxton Beale. & .50 \\
\hline & Mrs. Mary Stearns Burke. & .00 \\
\hline 199 & Emile Ashe. & .50 \\
\hline 202 & Mrs. Jno. R. Walsh & 1.00 \\
\hline & Genevieve Bothin & .50 \\
\hline & Yosemite $\ldots . .$. & 1.00 \\
\hline & London Smoke. . & .50 \\
\hline & Marin & .5 \\
\hline & Mrs. Rudolph Spreckels. . & \\
\hline & Pola Negri ...... & 1.00 \\
\hline & 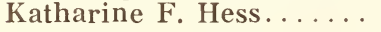 & 1.00 \\
\hline
\end{tabular}

\section{COLLECTION NO. 6}

\begin{tabular}{|c|c|c|}
\hline & Diener's American Beauty & \\
\hline & D. J. Whitney........... & \\
\hline 67 & Mrs. J. K. Armsby...... & 3.00 \\
\hline & Else Rose ........ & .75 \\
\hline & Sierra .... & 1.00 \\
\hline 174 & Atlantis & \\
\hline & Mrs. Leon Douglas. & 3.00 \\
\hline & Altamyra $\ldots \ldots \ldots \ldots$ & \\
\hline & Victory & 1.00 \\
\hline & Mr. H. A. Hyde. . & 1.00 \\
\hline & Martha Fernekes. . & \\
\hline & Yosemite & 1.00 \\
\hline & Pasadena & \\
\hline & Salome Jane & \\
\hline & California Giant .. & 00 \\
\hline & Nectarine & \\
\hline & Gladis Plath & \\
\hline & Katharine F. Hess . & 1.00 \\
\hline & Elf $(2$ bulbs $) \ldots \ldots$ & \\
\hline & Phylos & \\
\hline & Dr. Adolph Rosenthal . & \\
\hline & Bubbles & \\
\hline & & \\
\hline
\end{tabular}

Value .................\$29.25 One each of above for .....\$25.00

COLLECTION NO. 7

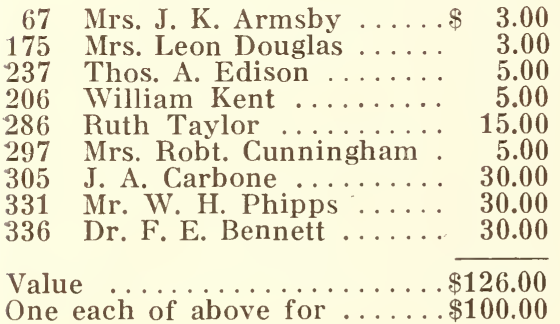

COLLECTION NO. 8

\begin{tabular}{|c|c|c|}
\hline 89 & Norma Krausgrill & 2.50 \\
\hline 96 & Richard Diener. & 5.00 \\
\hline 175 & Mrs. Leon Douglas ....... & 3.00 \\
\hline 184 & Alma & 5.00 \\
\hline 211 & Zoe & 3.00 \\
\hline & Yosemite & \\
\hline 220 & Eldiva. & 4.00 \\
\hline 234 & Belvedere & \\
\hline 241 & Victoria Fredericks & 0 \\
\hline 257 & California Giant ... & 3.00 \\
\hline 312 & Mrs. Richard Lohrman & 1.50 \\
\hline 320 & Esther Olson ......... & .00 \\
\hline & Mr. Henry Bechman .... & \\
\hline 337 & Virginian. & 4.00 \\
\hline & Thais Valdemar & 3.00 \\
\hline & Lagunitas $\ldots \ldots$. & 5.00 \\
\hline & Carmelita & 3.00 \\
\hline & Cattlaya & 3.00 \\
\hline
\end{tabular}

One each of above for ......\$ 50.00

\section{COLLECTION NO. 9}

276 Bernard Shaw ( $1 / 2$ size) $\$ \$ 5.00$

286 Ruth Taylor ( $1 / 2$ size) . 7.50

295 Blasco Ibanez ( $1 / 2$ size) . $\quad 5.00$

305 J. A. Carbone ( $1 / 2$ size) ‥ 15.00

331 Mr. W. H. Phipps ( $1 / 2$ size) 15.00

336 Dr. F. E. Bennett ( $1 / 2$ size) 15.00

338 Geraldine Farrar ( $1 / 2$ size) $\quad 37.50$

339 Henry Ford ( $1 / 2$ size) ... 25.00

Value .................. $\$ \overline{\$ 125.00}$

One each of above for . . . . $\$ 100.00$

\section{COLLECTION NO. 10}

269 Geisha ...........\$ 10.00

276 Bernard Shaw ......... 10.00

304 San Jose .............. 5.00

317 Lucia ............ 5.00

329 Moonbeam ............. 10.00

331 Mr. W. H. Phipps ..... 30.00

335 Mr. Henry Bechman .... 5.00

338 Geraldine Farrar ...... 75.00

339 Henry Ford .......... 50.00

Value ...............\$200.00

One each of above........ $\$ 150.00$

\section{COLLECTION NO. 11}

In Shades of Red

82 John Merle Coulter ....\$ .25

107 Heart of Juanita ........ .50

164 Capt. A. C. Baker ...... $\quad .05$

192 Victory .............. 1.00

203 Sir Roger Casement ... $\quad .25$

226 Pasadena .......... 1.00

Value $\ldots \ldots \ldots \ldots \ldots \ldots \ldots \$ 3.05$

One each of above for .....\$ 2.00 
RICHARD DIENER CO., INC., KENTFIELD, MARIN COUNTY, CALIFORNIA

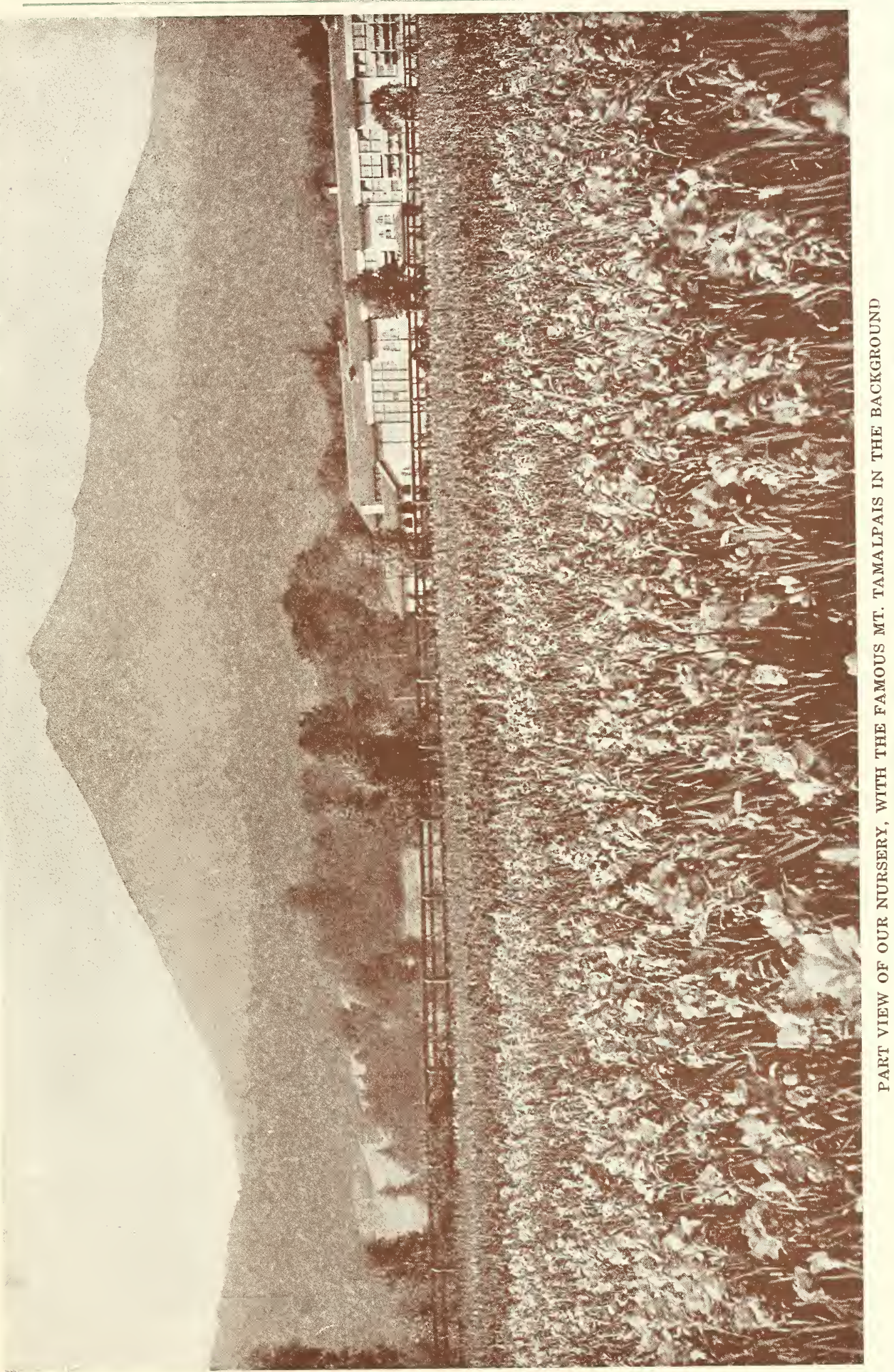

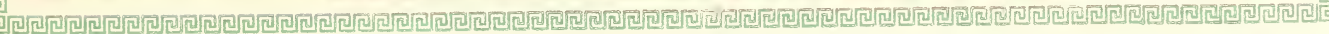


COLLECTION NO. 12

Ashes of Roses Color

145 Mrs. Cothran ........ . .25

186 Lillian Webb .......... .05

198 Ross Valley ............. .10

209 Genevieve Bothin ........ 50

221 London Smoke ......... $\quad .50$

332 Rose Ash .............. $\quad .50$

361 Carmelita .............. 3.00

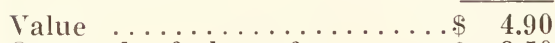

One each of above for .....\$ 3.50

COLLECTION NO. 13

White, Cream and White With Markings

19 Myrtle McNally .......\$ .25

22 San Anselmo .......... .10

90 Caroline Frear Burke ...

98 Mable Hubbard ....... $\quad .50$

117 Sierra .............. 1.00

166 Diener's White ........ 1.00

180 Mrs. Truxton Beale ..... $\quad .50$

199 Emile Ashe ......... $\quad .50$

200 Mr. H. A. Hyde ....... 1.00

Value .................\$ 5.10

One each of above for .....\$ 3.50

\section{COLLECTION NO. 14}

Lavender and Rose Shades

Sarah Lillie.......... .15

9 Sausalito ........... .50

13 Sunset ..............

101 Else Rose ............. $\quad .75$

130 Thomas T. Kent........ 25

173 Hawaii .............. 50

176 Miss Maud Fay.......... . .50

187 Costa Rica ............ .25

189 Elizabeth Gerberding. . . . .

196 Helen Todd ............ $\quad .10$

311 Gladis Plath............ $\quad .75$

333 Wistaria ........... .25

Value ............... $\overline{4.60}$

One each of above for.......\$\$ $\quad 3.00$

\section{COLLECTION NO. 15}

American Beauty Color and Magenta

23 Diener's American Beauty $\$ 1.00$

32 Fairfax ............... 15

220 Eldiva $\left(\begin{array}{lll}1 / 2 & \text { size }\end{array} \ldots \ldots \ldots .2 .00\right.$

281 Del Monte $\left(\begin{array}{l}1 / 2 \text { size }) \ldots . \\ 2.50\end{array}\right.$

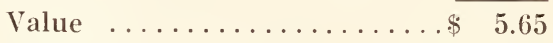

One each of above for .....\$ 4.00

COLLECTION NO. 16

Yellow, Salmon and Orange Shades

24 D. J. Whitney ........ \$ 1.00

31 Pres. C. C. Moore ..... .15

38 Jack London ......... . .25

39 Dr. Lincoln Cothran .... . .25

162 Mary Roberts Rinehart .. $\quad .20$

188 Mrs. Mary Stearns Burke .50

191 Mrs. H. E. Bothin ..... .25

198 Ross Valley ........... . 10

240 Salome Jane .......... 1.00

283 Nectarine ........... .50

293 Pola Negri ......... 1.00

333 Wistaria ........... .25

Value ..............\$ 5.45

One each of above for......\$ 3.75

\section{COLLECTION NO. 17}

Flame Color

205 David Starr Jordan .....

218 Yosemite ........... 1.00

232 Flame ............ 1.00

277 Beatriz Michelena ..... 4.00

283 Nectarine ...............50

354 Thais Valdemar....... 3.00

value $\ldots \ldots \ldots \ldots \ldots \ldots \ldots \$ 9.75$

One each of above for $\ldots \ldots \$ 7.50$

Mr. Richard Diener, Kentfield, Cal.

Detroit, Mich., January 17, 1923.

Dear Sir:-My small order of Glads was received. It pleased me immensely. Most of the corms measurcd over three inches across. This order convinced me that you give much attention and consideration not only to large orders, but to small ones as well; since it consisted only of one corm of a kind, and in several cases of a small one.

About last year's order, will say that Mrs. Wm. Kent, American Beauty, John Merle Coulter, Mary Stearns Burke, Mrs. Willard Richardson gave each over a hundred cormels and those fron Anna Eberius and irrs. Willard Richardson were exceptionally large.

Very respectfully,

ADAM S. STEINHAUER, M. S.

Richard Diener Co. Inc.

Midland, Michigan, Aug. 30, 1923.

Dear Sirs:-I had some wonderful blooms from your bulbs and took scven prizes at the Saginaw show, three of them being firsts.

My spike of American Beauty was very much admired, and according to comment was better than had been shown in previous years. California Giant is just opening with a $5^{\prime} 6^{\prime \prime}$ spike and is still growing. I am looking forward to the receipt of your new catalog, as I shall wish to try out a few of your recent and more of your older varieties. I am looking forward to the time when Henry Ford, Geraldine Farrar and W. H. Phipps will come within the range of my purchasing power. 
RICHARD DIENER CO., INC., KENTFIELD, MARIN COUNTY, CALIFORNIA

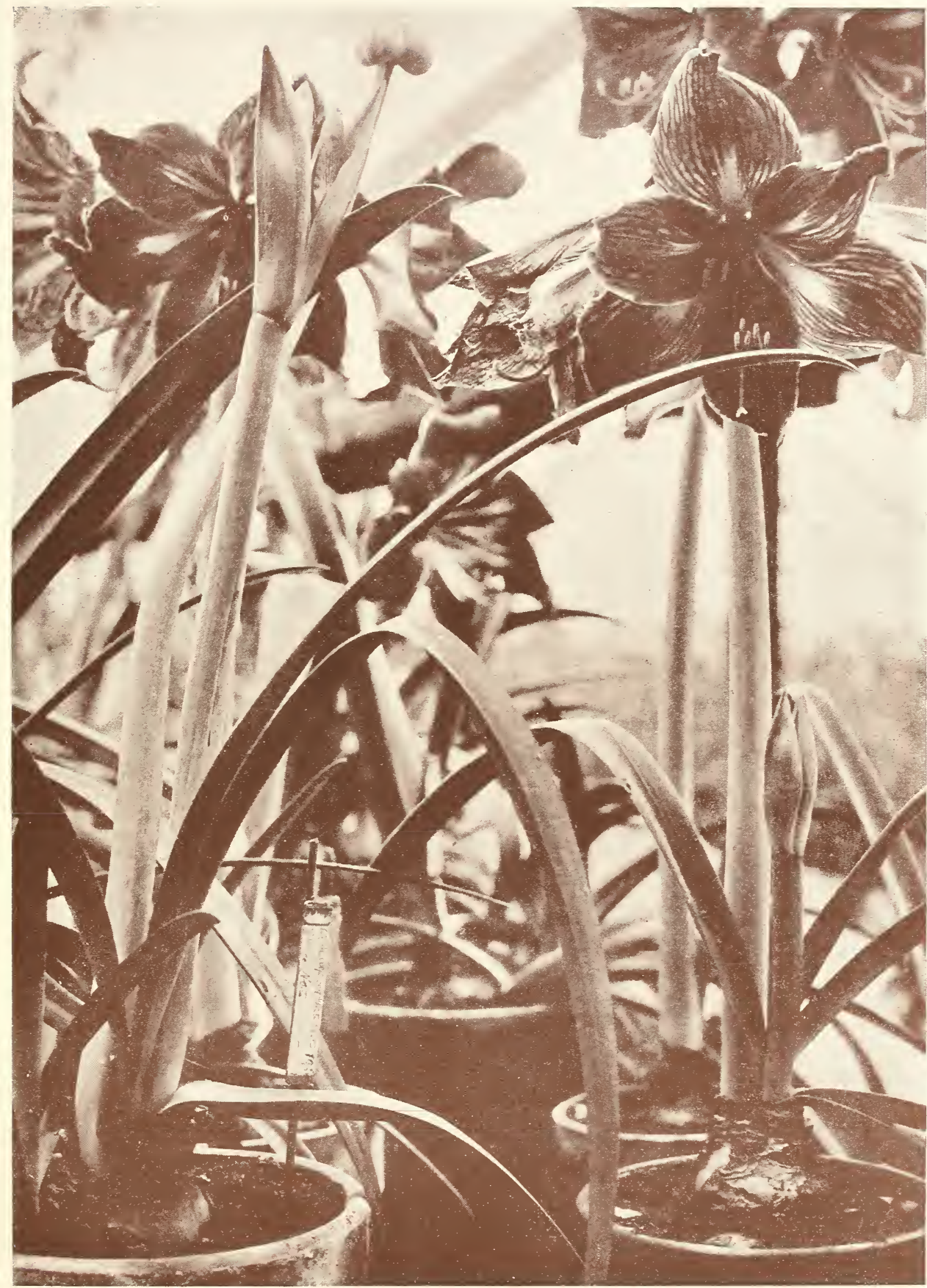

NOTE-WHILE PLANT HAS SEED RIPE, NEW FLOWER SPIKE IS AFPEARING 


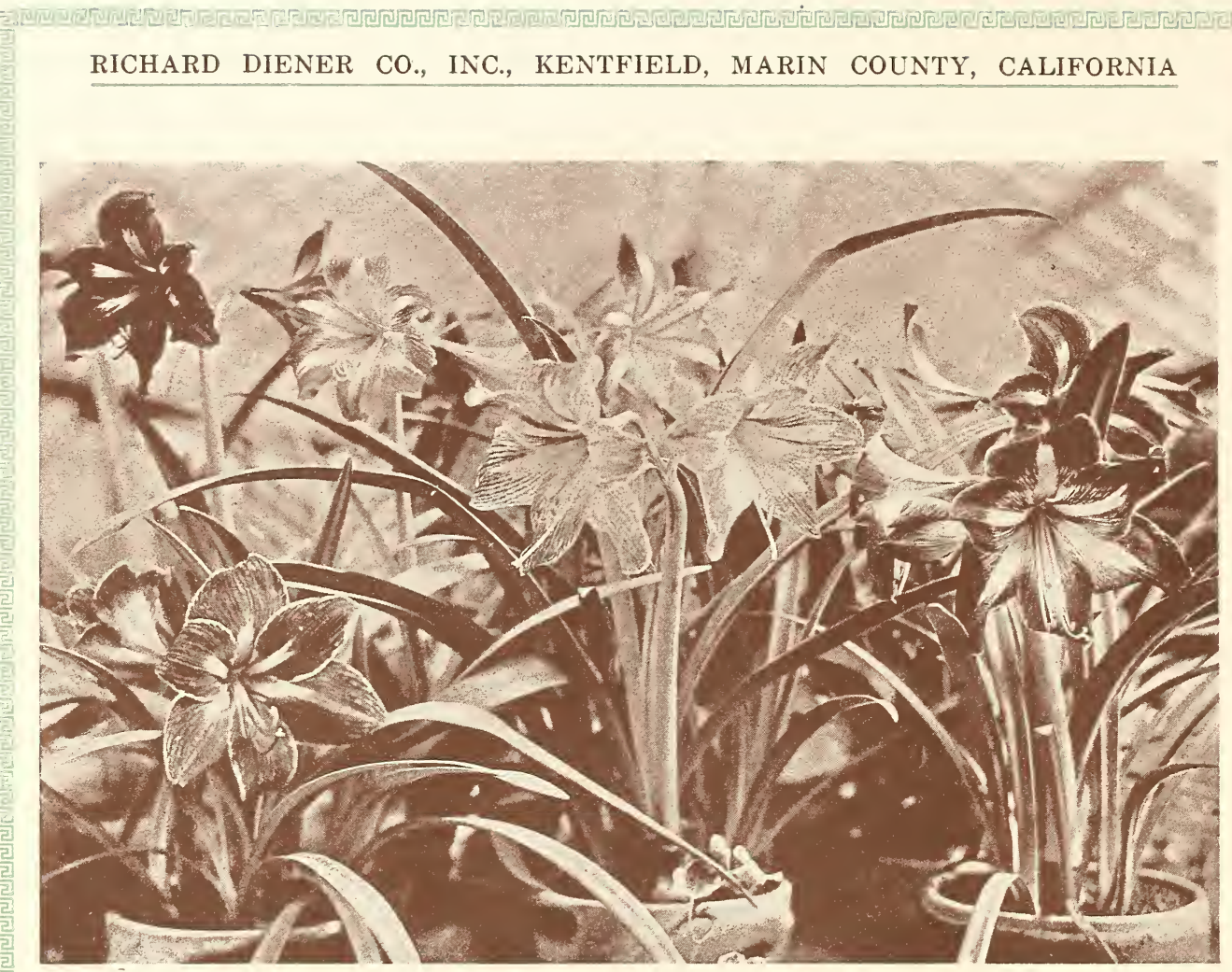

\section{Diener's Mammoth Amaryllis Hybrids}

For years Mr. Diener has been working on Amaryllis to enlarge and enrich the coloring, and we have now a good stock on hand, the colors ranging from white to all shades of pink; red to the deepest scarlet; the most brilliant and dazzling colors imaginable. The flowers are from 10 to 14 inches in diameter and there are mostly four flowers to the stem. Unlike the old Amaryllis, which flowers only once a year, these new hybrids bring a spike every 3 to $4 \mathrm{months}$. They are very valuable for cut flowers. If the pollen is removed when the flowers open, they will last for several weeks.

Diener's Mammoth Amaryllis Hybrids are the coming big show objects of the future. Anyone who has some space in the greenhouse should raise a batch of these seedlings. Cultivation is simple, as they grow very easily. Soil suitable for a geranium or Fuchsia is all right for these Amaryllis. They must have very little shade when under glass, and during the summer kept out in the open in the full sun. When bulbs are larger, it is necessary in planting that just the roots be placed in the ground, the whole bulb must be above the ground. Another point of importance is: Do not water while the ground is wet. Wait until soil gets dry.

These Amaryllis should be kept under continual cultivation all the year around; never rested or kept dry, the nearer the temperature is kept to 70 degrees during the night, the more, and the better they will flower.

\section{HOW TO SOW OUT AMARYLLIS SEED}

Sow out in boxes or seed pans (in greenhouse or hotbed), containing finely sifted sandy leaf mold to a thickness of three inches. Smoothen out surface of soil evenly and wet thoroughly. lear mold the same Spread seeds closely together (so close that they touch one another), and cover with the same
soil just sufficient to hold them down. Keep moist but not too wet. If air is too dry, cover with glass. In case of very hot sun, care must be taken that glass is covered with a sheet of newspaper, in order to prevent heat forming between glass and soil, which will cook the young plants.

Young plants will come up in about three weeks and should remain in the same box for another two or three months. Then replant in three inch pots, or in boxes about two inches apart. Seedlings, when given the proper care, will commence to flower 18 months after sowing. Seed can be sowed out at any time of the year.

15 seeds $\$ 1.00,100$ seeds $\$ 4.00,1000$ seeds $\$ 35.00$

\section{AMARYLLIS BULBS-DIENER'S HYBRIDS}

Young stock ready to flower, $\$ 2.00$ per bulb, $\$ 20.00$ per dozen; $\$ 3.00$ per bulb, $\$ 30.00$ per dozen. Other bulbs of larger size at $\$ 5.00, \$ 7.00, \$ 10.00$ and $\$ 15.00$ each, according to the size of the flowers. Prices of larger quantities on request. 


\section{Petunias}

\section{DIENER'S RUFFLED MONSTERS}

Diener's Ruffled Monsters are so well known to amateurs and professionals alike, and have been shown at almost every flower show in the United States, Canada, Australia and New Zealand, as well as in Europe, that they are proving to be the greatest sensation among flower novelties.

The varieties that we are sending out this year have again been greatly improved over those of former years, and have some new colors added as well as the Double Varieties, that we are offering for the first time.

Thousands of visitors from all parts of the Globe have visited our place, expecting to see something great in Petunias, and were more than surprised at the high perfection to which we have brought them; and rightly so, the old petunias were considered by everyone to be the commonest of garden flowers, while the new Ruffled Monsters have been so greatly improved, that they stand on an equal level with the orchid in beauty and coloring.

Our aim is to give our customers the finest Petunia Seed obtainable, and we will go to any trouble and expense to produce it. All of our seed is raised in lath houses, where insects are excluded and pollenation is done artificially by hand. This is the only way to get seed true to type. Besides this, our climate is the most ideal in the world for producing perfectly ripened seed, as we have no rain during the time that it is setting. Most all of the Petunia Seed produced today is raised in the open, and the pollenation is done by insects. Seeds raised in such a way are inferior.

As Petunias revert to the old type continually, it does not pay anyone to raise his own seed, as in most cases the results will be poor. It requires long years of experience to know the habits of Petunias and to overcome the effects of reverting. No matter how small a quantity is needed, you will find you will do better by buying our seed, than by raising your own.

In shipping seed, we take the utmost care to have it arrive in perfect condition. We take pains in packing so that it will be moisture-proof, and at the same time will not be crushed in transit.

Most of the other flowers have their seasons, and bloom only once a year, but not so with the Petunias. They flower continually, all the year round, making them of still greater value. They are very good as a cut flower, but many people are not aware of the fact. When put into water, the stems of the cut petunias will last from 2 to 3 weeks and new flowers will bloom while the old blossoms are dropping off.

\section{Instructions for Sowing Petunia Seeds}

Prepare a seed bed by taking two parts of any light compost or leaf mold thoroughly mixed with one part of clean sand. Sift all through a fine sieve. With this mixture, fill flats or seed pans, pressing down the soil to a flat surface within two inches of the top. Give a thorough soaking. After the water has seeped into the soil, sprinkle the Petunia Seed evenly over this surface, but do not cover with the least bit of soil. To prevent attacks, however, of fungi, which might easily kill the young Petunia Plants, sprinkle the newly sown flats with charcoal dust. Place a clean pane of glass over the flats or seed pans, and in the daytime, if reached by the sun's rays, place a single sheet of newspaper over the glass to prevent burning. At night and in dark weather remove this sheet of paper. Avoid carefully all excess of moisture and have pans near glass in well ventilated atmosphere. As soon as the seed sprouts, (say in 8 to 10 days after sowing, if kept at a temperature of 60 degrees) take off the pane of glass entirely. The young plants should be transplanted as soon as possible into flat boxes, spacing them an inch apart.

If this seed is handled right, every kernel will germinate, as they are cleaned by a process where only fertile kernels are selected. Ants will carry off the seeds if they have any access to the same. Snails will eat the voung seedlings, a great many in one night. For this reason, seed boxes should be placed so that snails and ants are unable to reach them. Most of the loss of the seed occurs in this way.

We would suggest in this case that the seed box be placed on small blocks of wood, the blocks resting in jars or pans, the latter containing either oil or water. The ants or snails are unable to cross the oil or water, and consequently cannot reach the seed. 


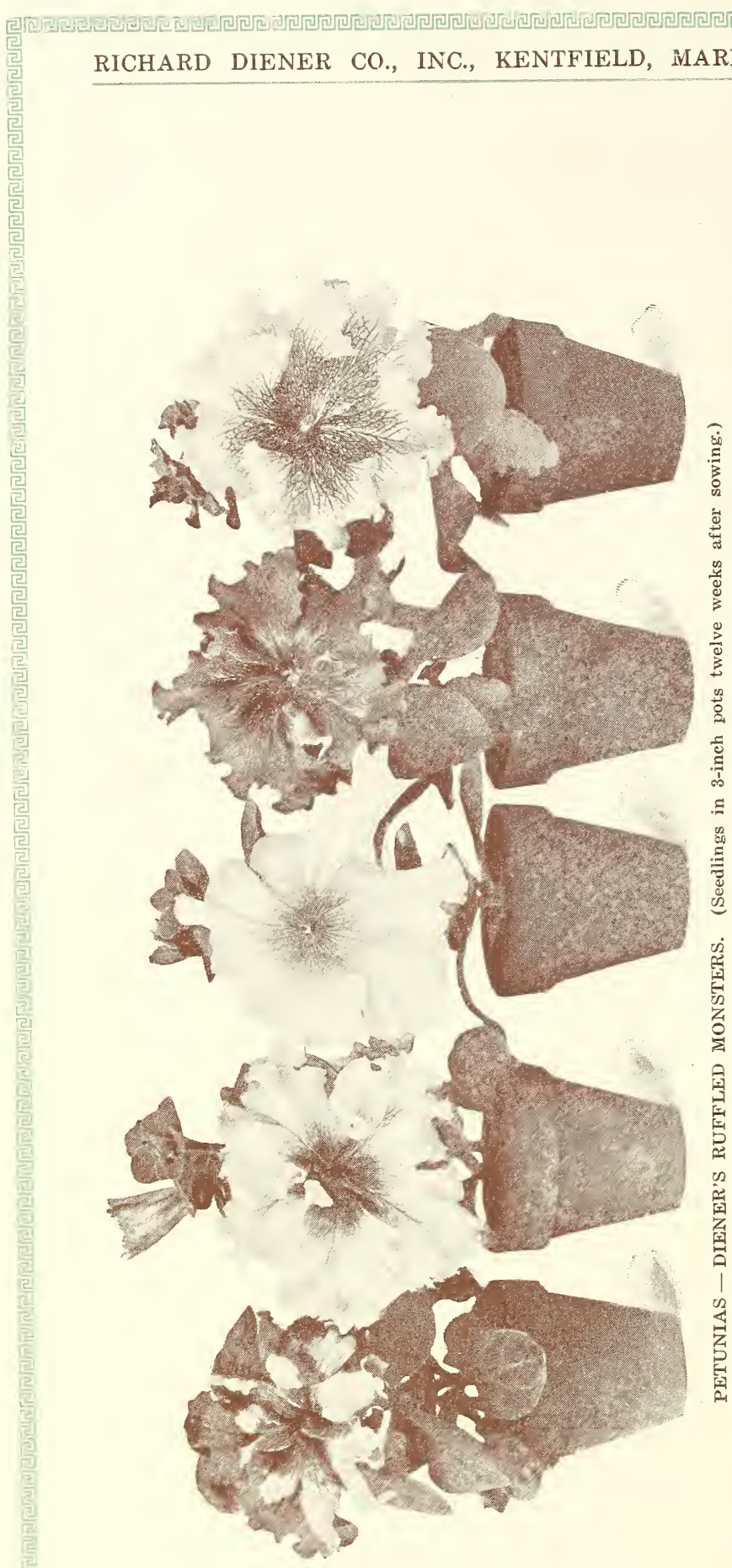

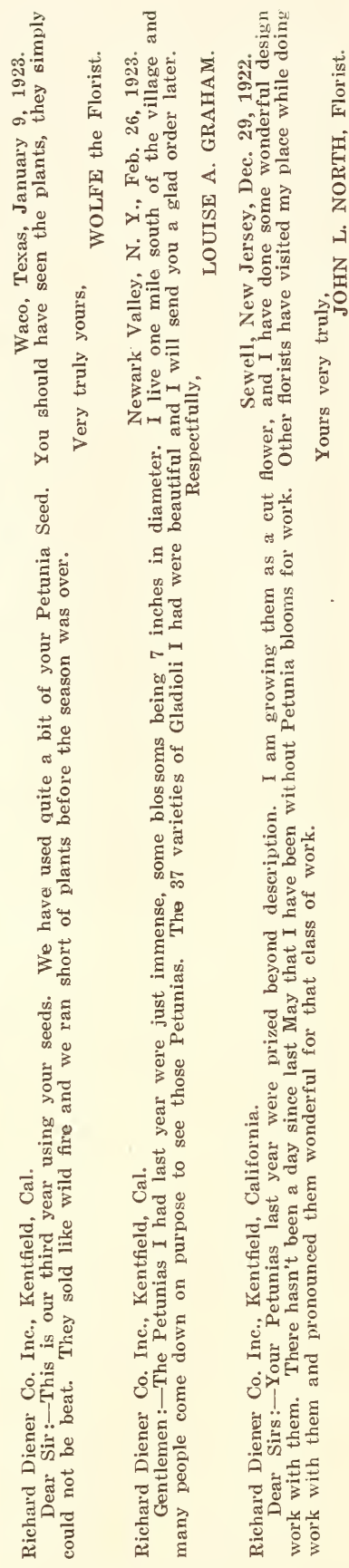




\section{Diener's Ruffled Monsters}

When ordering, order by number.

No. 1. Pink: Strong veined center. The size of this variety is from 6 to 8 inches in diameter and the color combination is most exquisite, rivaling the orchid.

No. 2. Red: With strong veined Mahogany and Black center. Average flower from 5 to 7 inches in diameter.

No. 3. Variegated: Colors appearing in checks, blotches and stripes of red, pink and white. Centers strong veined. Size 5 to 7 inches in diameter.

No. 4. White.

No. 5. Red with Black center: The outside part of the flower is red with the center coal black.

No. 6. Pale Lilac Pink: Heavily ruffled and center strongly veined; size 4 to 5 inches in diameter.

No. 7. Red and White variegated: Large Yellow center.

No. 18. Heavy ruffled: Outside white with blotches and checks of magenta appearing on the edges of the petals. Large yellow center. Size of the average flower is from 4 to 5 inches.

No. 20. Mauve: Center heavily veined with Royal Purple. One of our most exquisite varieties. The average flower is 5 inches in diameter.

No. 8. Ruffled Monsters Mixed. All colors of the above mentioned varieties evenly mixed.

THE FOLLOWING VARIETIES ARE SOMEWHAT SMALLER THAN THE RUFFLED MONSTERS.

No. 9. Flesh Pink Frilled: A most beautiful variety, with edges of the flowers heavily ruffled. The size of the flower is from 3 to 4 inches in diameter.

No. 10. White Frilled: Snow white with flowers 3 inches in diameter. Plants of very dwarf growth.

No. 11. Blue or Purple.

No. 12. Apple Blossom: Pale Apple Blossom pink. Size 3 inches in diameter. Plants of very dwarf growth. With frilled edges.

No. 13. All varieties mixed including Ruffled Monsters and Pink Glory.

No. 14. Diener's Pink Glory: The greatest novelty in Petunias, pure flame rosepink, without a trace of purple. Flowers measure 3 to 4 inches in diameter and are slightly ruffled. Plants grow to a height of from 12 to 15 inches. A very compact grower and is continually covered with flowers. This variety is destined to become the great bedding Petunia of the future.

No. 15. Blue and White: The flowers of this variety are of medium size. The color combination of the flowers is blue and white, the same appearing in blotches, stripes and speckles. Especially adapted for window boxes or hanging baskets.

No. 16. White Giant: Snow white. Flowers of the largest size, 5 to 6 inches in diameter. The seedlings of this variety come $95 \%$ true. As this seed is very scarce, we will limit it to one package to a customer.

No. 21. Deep Violet: A velvety flower with a delicate silver seam around the entire edge. Plants grow very sturdy and compact.

Any of the above varieties, $50 \mathrm{c}$ a package.

Between two and three hundred seeds in a package.

We do not sell less than full packages.

\section{Wholesale Prices - Petunia Seeds}

Diener's Ruffled Monster Petunia Seed Mixed and in Separate colors including Numbers $1,2,3,4,5,6,7$ and 8 .

$1 / 16$ Ounce.............\$ $7.50 \quad 1 / 4$ Ounce............. 25.00

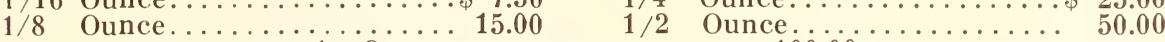

$$
1 \text { Ounce.................... } 1000
$$

Diener's Blue or Purple and Flesh Pink Frilled, Numbers 9 and 11.

$1 / 16$ Ounce.............\$5.00 1/4 Ounce ...........\$ 20.00

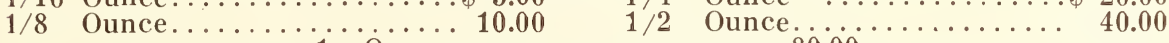

1 Ounce................ 80,00

Diener's Pink Glory No. 14 (Pure Flame Rose-Pink).

$1 / 16$ Ounce.............\$\$ $8.50 \quad 1 / 4$ Ounce.............\$ 32.50

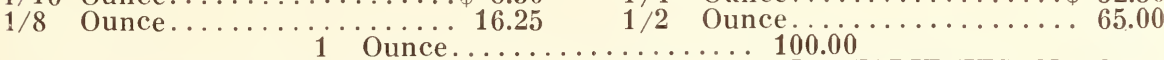

Note: Prices on larger quantities of DIENER'S DOUBLE VARIETIES, Numbers $17,22,23,24$ and 25 mav be had on request. 


\section{Double Varieties}

No. 17. Pale Sulphur Yellow: Flowers single and double with heavy frilled edges. Entirely new. It being the first clear yellow. Size from 3 to 4 inches.

Price per package........................ 50

No. 22. Rose Color Double: The brilliancy of this flower makes it stand out above everything else. Very showy and striking, wonderful for massing in beds or borders. Very free blooming. Plants are of very dwarf growth, reaching a height of only 6 to 7 inches. This variety comes $50 \%$ double. Flowers 2 inches in diameter.

Price per package........................... 50

No. 23. "ROSA BONHEUR": Pale amaranth pink. Flowers double, averaging 2 inches in diameter. Of very dwarf growth. Makes an attractive bed or border. Flowers bloom constantly.

Price per package......................... 50

No. 24. GIANT DOUBLE FRINGED MIXED: Colors ranging from snow white to all the shades of pink and mauve, purple and dark red, the lighter shades predominating. The double flowers are very large and perfectly formed, some being beautifully fringed, while others are heavily ruffled and fluted. This mixture is the very finest that can be had.

Price per package........................\$1.00

No. 25. DOUBLE FRINGED MIXED: Flowers double and of all colors imaginable. Plants sturdy and erect, yielding a profusion of brilliantly marked flowers, some being fringed, while others are fluted and frilled, having lace-like edges. The pastel coloring in these blossoms rivals the orchid. Price per package...................... 50

\section{Pansy Seed}

\section{Giant Excelsior}

A most beautiful Pansy Mixture. They contain every color in the rainbow, and the single flowers are of the very largest size. They are ruffled and fluted, and for bedding purposes are unexcelled.

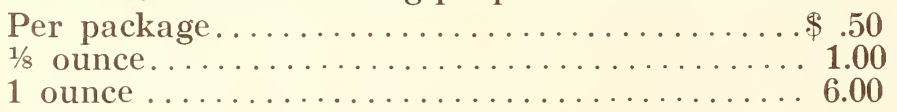

\section{Oregon Giants}

A pansy strain of immense size and of wonderful colors.

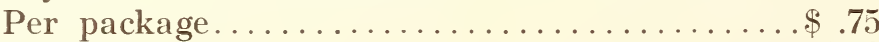

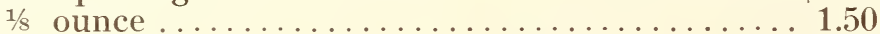

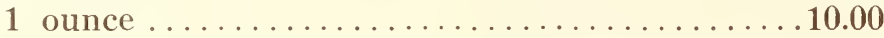

\section{Directions for Sowing}

Any rich, sandy compost soil or old hotbed soil is good to sow the seeds out in, either in boxes or hotbeds. Level off the ground and sow seeds thickly. Cover to the thickness of the seeds with the same soil, and keep moist. As soon as the seedlings have from 2 to 3 leaves, transplant them to two inches apart either in boxes or in the open. 
RICHARD DIENER CO., INC., KENTFIELD, MARIN COUNTY, CALIFORNIA

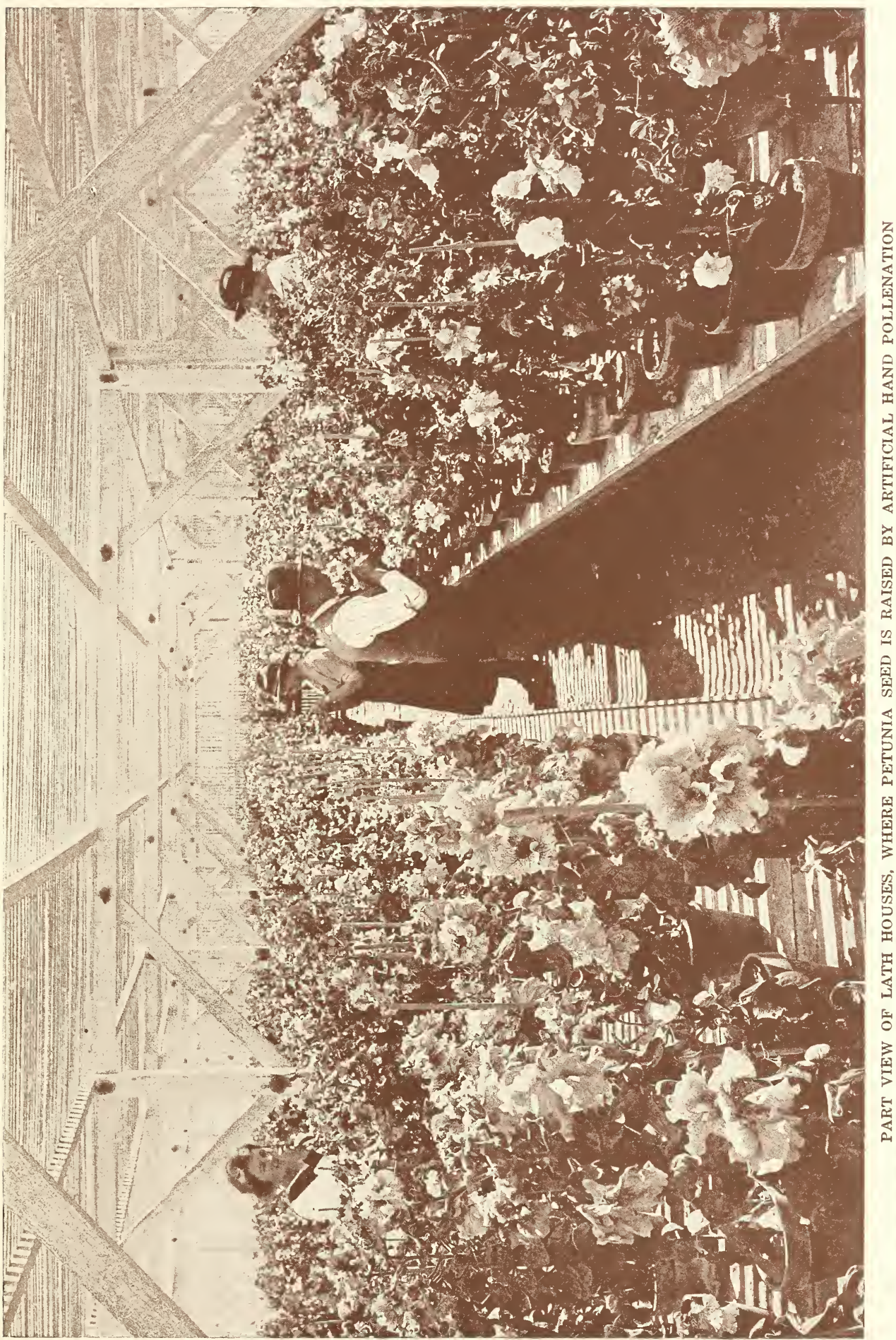




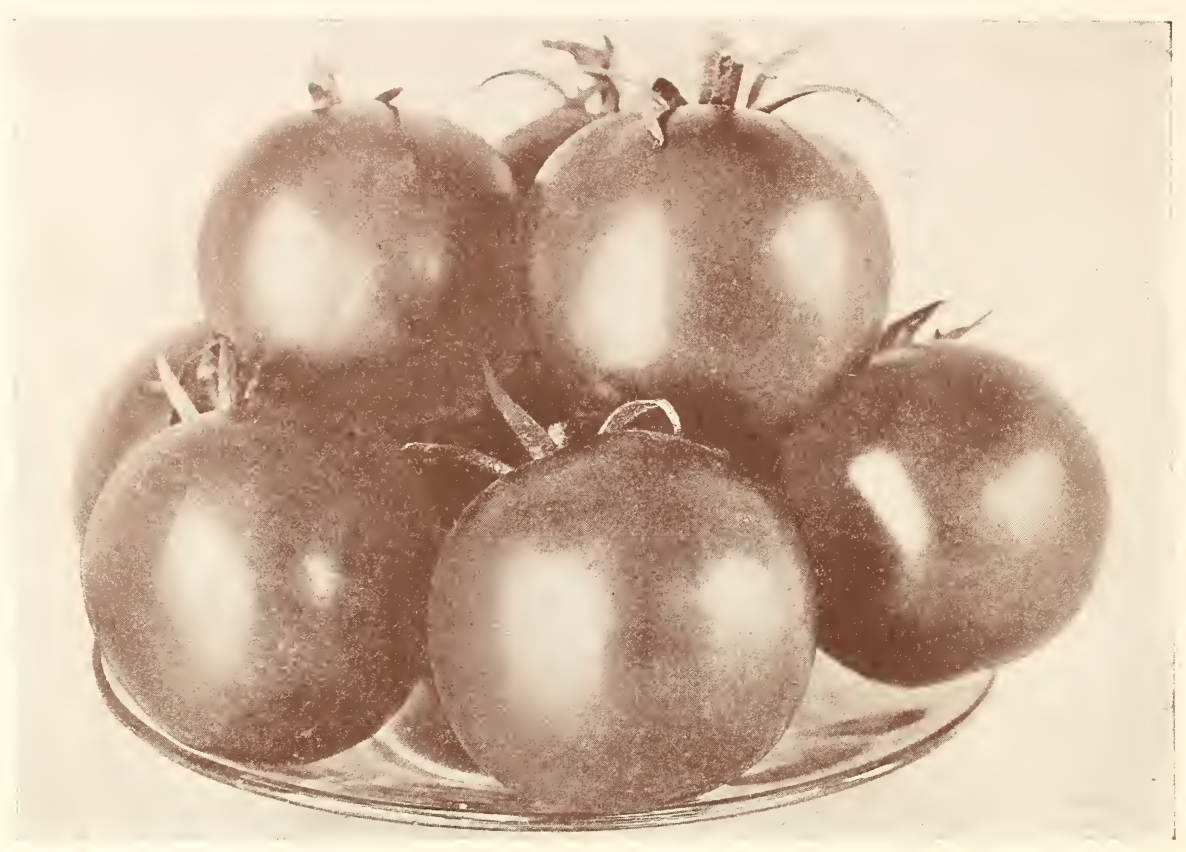

\section{The Pepper Tomato}

An entirely new fruit, a cross between a bell pepper and tomato. Many of our visitors this summer have tested this fruit, and all invariably declared the same very much richer in flavor than the tomato. The shape of the fruit is oval, and shows the corners like the bell pepper. The color is scarlet and very different from the tomato. Flesh is of a beef steak color, the inside is fully filled out, but shows more the pepper formation, single fruit weighing up to one pound. The growth of this plant is very vigorous, and should be planted 6 to 8 feet apart. The leaves show no signs of any disease whatsoever. It is an extra early ripening and free-bearing variety. We predict a great future for this tomato, as it is perfect in every respect.

Price per package. $1 / 2$ oz.
$1 \mathrm{oz}$.

$1 \mathrm{lb}$.

\section{Carters Sunrise Tomato}

A very early but small variety; round as an apple. It grows in clusters, about ten tomatoes in one cluster. Six tomatoes weigh about one pound. The flesh is a dark red and of good taste. It is a very heavy yielder and one of the best varieties for forcing under glass.

Per package...................... 25

\section{The Burbank Tomato}

This is an extra early variety. The flesh is a deep red and of good flavor. The bushes reach a diameter of 4 feet only and are loaded with fruit.

$$
\text { Per package................... .25 }
$$




\title{
The Diener Tomato
}

\author{
(Blight Resisting)
}

AWARDED GOLD MEDAL at the California Industries and Land Show, Oct. 5, 1919

This tomato was originated by Richard Diener in 1917, and has proven in every respect far superior to anything existing now. The size of the first fruit reaches the weight of 3 pounds, but the average fruit weight is about one pound, is perfectly filled out and does not contain any hollows like so many other varieties and the surface is as smooth as an apple.

The flesh is more solid than any other Tomato now known, and for dehydrating is a most valuable variety. It is a magnificent dark red shade, which makes it very attractive; it is very sweet and free of the acid taste. For catsup it is unexcelled, as it makes a very high red color; contains very little seed. The skin is a deep orange red.

The yield is enormous and is not reached by any other variety in existence. The most astonishing thing about this tomato is that it has proven to be absolutely blight resisting. It also endures drought better than any other variety on the market today.

The growth of this variety is strong and healthy, and plants have a diameter of 6 to 8 feet.

For long distance shipping purposes this variety is ideal.

All the seed we send out is absolutely true, as we raise it from cuttings of the original plant, and as the whole field is of the same plant, it is impossible for side crossings. For this particular reason we advise you to always buy your seed direct from us in order that you are sure to have the same quality that you have now, as seeds raised from seedlings will vary and not be the same.

This tomato has been grown all over the United States and Canada, and the reports from everywhere are highly satisfactory, as you will note when reading testimonials.

\section{Diener Tomato Seed}

\section{Cutting Raised Seed}

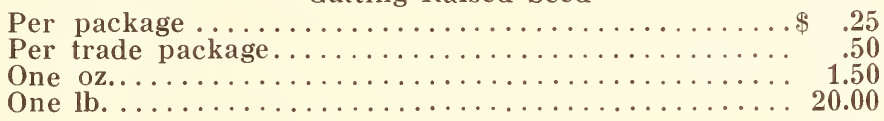

Seedling Raised Seed

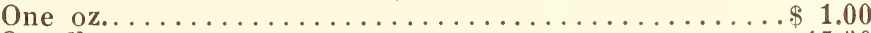

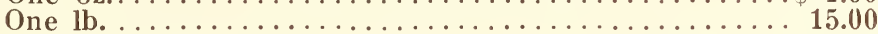

INSTRUCTIONS FOR SOWING SEED

Seeds should be sowed in boxes during the months of January and February. If possible, replant once about two inches apart in light compost of leaf mold thoroughly mixed with one part of sand. Keep indoors in a frost-protected place. As soon as danger of frost is over put out into the open field in the full sun. Have ground plowed from 10 to 12 inches deep. If set in a light or sandy ground, plant six feet apart, and if in heavy ground, eight feet apart. in a light or sany ground, plant six feet apart, and if in theavy ground, Keep the ground free from weeds and anything on the vines or stake the plants. Let the vines grow their natural way, as in this way the growth will give the fruit even shade and the sun will not cook it. Do not be afraid of the fruit rotting when on the ground, as this variety will not do so; even rainy weather will do no harm to the fruit. This tomato can be washed without being cracked by the water.

Richard Diener Co. Inc., Kentfield, Cal

Flournoy, Calif.

Dear Sirs:-I bought some seed of you several years ago, and my sister-in-law took some to the Tehama County Fair. Three tomatoes weighed 15 pounds. They are surely a fine tomato. Please send your catalog.

Yours very respectfully,

MRS. J. B. OSBORN.

Richard Diener Co. Inc.

Hollister, Cal., January 8, 1923.

Dear Sirs:- It might be of interest to you to know that I was presented with 8 acres of your plants last year and had a most satisfactory yield. Sold 101 tons and gave several tons away. Did not irrigate. Largest tomatoes weighed 2\% lbs. each. Many boxes of 45 tomatoes weighed 60 lbs. each. Cannery was always pleased to see my truck unload. Was not docked once. Many vines yielded over two boxes to a picking.

HERBERT A. SCHULZE. 


\section{Dahlias}

The following is a list of new Dahlias that we are offering for the first time. They are the results of many years of labor, and are crossings of the very finest varieties in existence. They are the cream of fifty thousand seedlings.

The main object in producing these varieties was to get long wiry stems, which the majority of the finest Dahlias now on the market are lacking. Our new varieties have great long, wiry stems, and are of extremely sturdy growth. The sizes of most all of them are the very largest so far produced. Their colorings are the acme of perfection.

These creations will equal, in the Dahlia World, what the Diener varieties of Gladioli stand for in the Gladiolus line.

We are sending out young green cuttings out of three inch pots the latter part of May. Only those varieties, for which the dozen price is given, may be had in quantity. Others will be limited to one of each to a customer.

\section{NOTE}

As there are thousands of named varieties on the market today we are listing ours according to number, in order to avoid using names which may have already been given some other varieties.

DIENER NO. 1.

An immense Chrysanthemum Type Dahlia of real ivory white with a pink underground, sliading. lighter toward the center. Because of its delicate color, fine foliage, long, wiry stems, it is a great contribution to the Dahlia Kingdom.

DIENER NO. 2.

$\$ 10.00$ each

Flame grenadine red with the extreme outer petals tipped with gold. Petals fold back very regularly, showing a very neatly folded center of red. This bloom resembles a large flaming ball, and is extremely striking and attractive. Stems long and rigid. A distinctly beautiful variety, admired by every visitor to our place.

$$
\$ 3.00 \text { each } \quad \$ 30.00 \mathrm{doz} \text {. }
$$

DIENER NO. 3.

A semi-cactus of great size. Bright Picric yellow, no markings. Petals long and curly, sturdy growth and enormously free blooming. Long, strong stems.

\section{$\$ 3.00$ each}

DIENER NO. 4.

This exquisitely beautiful semi-cactus is of bright picric yellow. The petals are of good substance, incurved and twisted so that each blossom is a striking bouquet in itself. Long, wiry stems. One of the finest in its class. Hot and dry weather does not affect this variety. Very large. One of the finest varieties ever produced. $\$ 5.00$ each

$\$ 50.00$ doz.

DIENER NO. 5.

Another beauty of the semi-cactus class. A very odd color combination. Amber-buff witl old rose and ruby shadings. Long twisted and curling petals. Good stems and a free bloomer.

DIENER NO. 6. $\$ 3.00$ each

Splendid variety of Champagne color shading into yellow at the center. This is an ellormous bloom, each petal being very large and folded back, producing a large, round mass of color. Long, rigid stems. Should be in every collection.

DIENER NO 7. $\$ 10.00$ each

Snow white with faint touch of lemon in the center which gradually grows lighter as the bloom

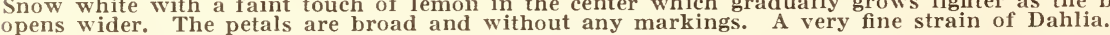

DIENER NO. 8. $\$ 5.00$ each

One of the most beautiful decoratives yet produced, of pure Chalcedony yellow. A most delicate One of the most color. Very long petals, some having pointed tips and folded together, whec others are of its class. divided and cut out. Center petals very closely folded together. A perfect specimen of its class.
The flowers are borne high above the foliage on long, rigid stems. Perhaps the largest in existence.

DIENER NO. 9 $\$ 10.00$ each

Cream background, petals colored with pinkish lilac, growing into a deeper shade of mallow purple in the center. Tips of petals are divided, giving the flower a beautifully fringed effect The stems are extra fine and long, and the blooms last well when cut.

$$
\$ 10.00 \text { each }
$$




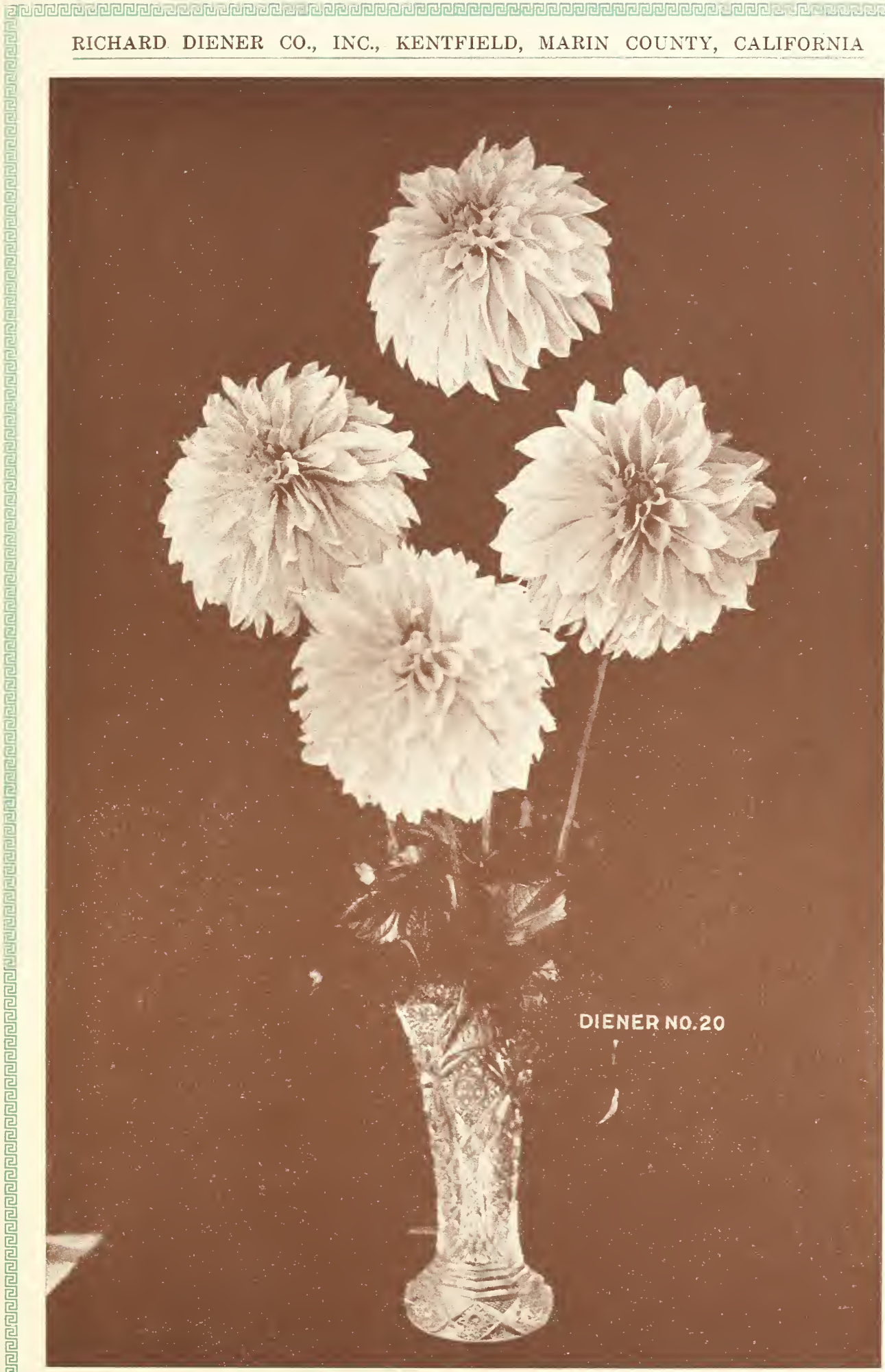


DIENER NO. 10.

A most striking combination of flaming coral and buff. The larger part of the flower is an intense coral and very iridescent, while the two outer rows of petals are tipped with straw. Petals are long and folded back, forming a large, flat flower. Good foliage and healthy grower. Stems very long and tough.

DIENER NO. 11.

\section{$\$ 5.00$ each}

A large blossom of Dahlia Purple with maroon shading. A well rounded bloom with petals very symmetrical. Deep colored center. Very long, wiry stems. An exquisitely beautiful specimen of the Dahlia World.

DIENER NO. 12.

$\$ 5.00$ each

A many-petaled Peony of pure Magenta with no markings. Petals are notched and cut out so that each one has three points, giving a wonderfully fringed effect. A most unique creation which should be in every collection.

\section{$\$ 5.00$ each}

DIENER NO. 13.

A most pleasing blossom of rose pink with straw color shading. Outer points show traces of lilac. A large, flat bloom, having long, broad petals arranged symmetrically. Good cut flower variety. Fine foliage and long, strong stems.

\section{$\$ 5.00$ each}

\section{DIENER NO. 14.}

An ellormous bloom of velvety carmine. No markings. Petals are long and twisted. Center practically covered with smaller petals, so that the blossom gives a glorious red effect. Fine foliage and strong growth.

DIENER NO. 15.

$$
\$ 5.00 \text { each }
$$

Champagne tinged with pinkish lilac. An unusually soft and well-blended color. Petals very broad and evenly arranged. A good, full decorative. Stems long and rigid.

\section{$\$ 5.00$ each}

DIENER NO. 16

A rosy pomegranate color with tips of petals shading into old rose. Petals of extra fine substance. Flowers immense and borne on long, rigid stems. An excellent cut flower variety.

DIENER NO. 17.

$$
\$ 5.00 \text { each }
$$

This very attractive Decorative of Bordeaux, tipped with Dahlia purple, very iridescent. Large, broad, open petals. Good long stenis.

DIENER NO. 18.

A very beautiful Dahlia of the Decorative type, rosy pink underground, overlaid and striped with Anaranth pink. Very free blooming. Sturdy stems.

DIENER NO. 19.

$\$ 3.00$ each

An exquisite combination of sulphur yellow and shell pink. A Decorative of great merit. Petals broad with slightly rounded tips, each being outlined with a faint pink line, so that every petal stands out individually. Flowers very hardy and set on lolg, sturdy stems. One of our best.

DIENER NO. 20. $\$ 10.00$ each

Large Decorative of fine form with very broad petals. Ground color is white with purplish lilac coloring. Long, strong stems. This Dahlia is perfect in every respect. Immense in size.

\section{$\$ 10.00$ each}

DIENER NO. 21.

A large full blossom of Vandyke red. Petals long and open, and of extremely heavy texture. Free blooming and of splendid growing habits. Good, strong stems.

$\$ 5.00$ each

DIENER NO. 22.

Large open flower of velvety texture. Color is ox-blood red, very striking. Petals broad and wide open. Fine foliage and flowers held erectly on very long stems.

\section{$\$ 5.00$ each}

DIENER NO. 24.

A very fine semi-double variety. Amaranth pink overlaid with Tyrian pink. Good, broad petals. Very free blooming. Good foliage and stems.

$$
\$ 3.00 \text { each }
$$

DIENER NO. 25.

Another of the semi-double variety. Real mallow purple with wide open petals. Iarge blossom, very free blooming. Long, wiry stems. 


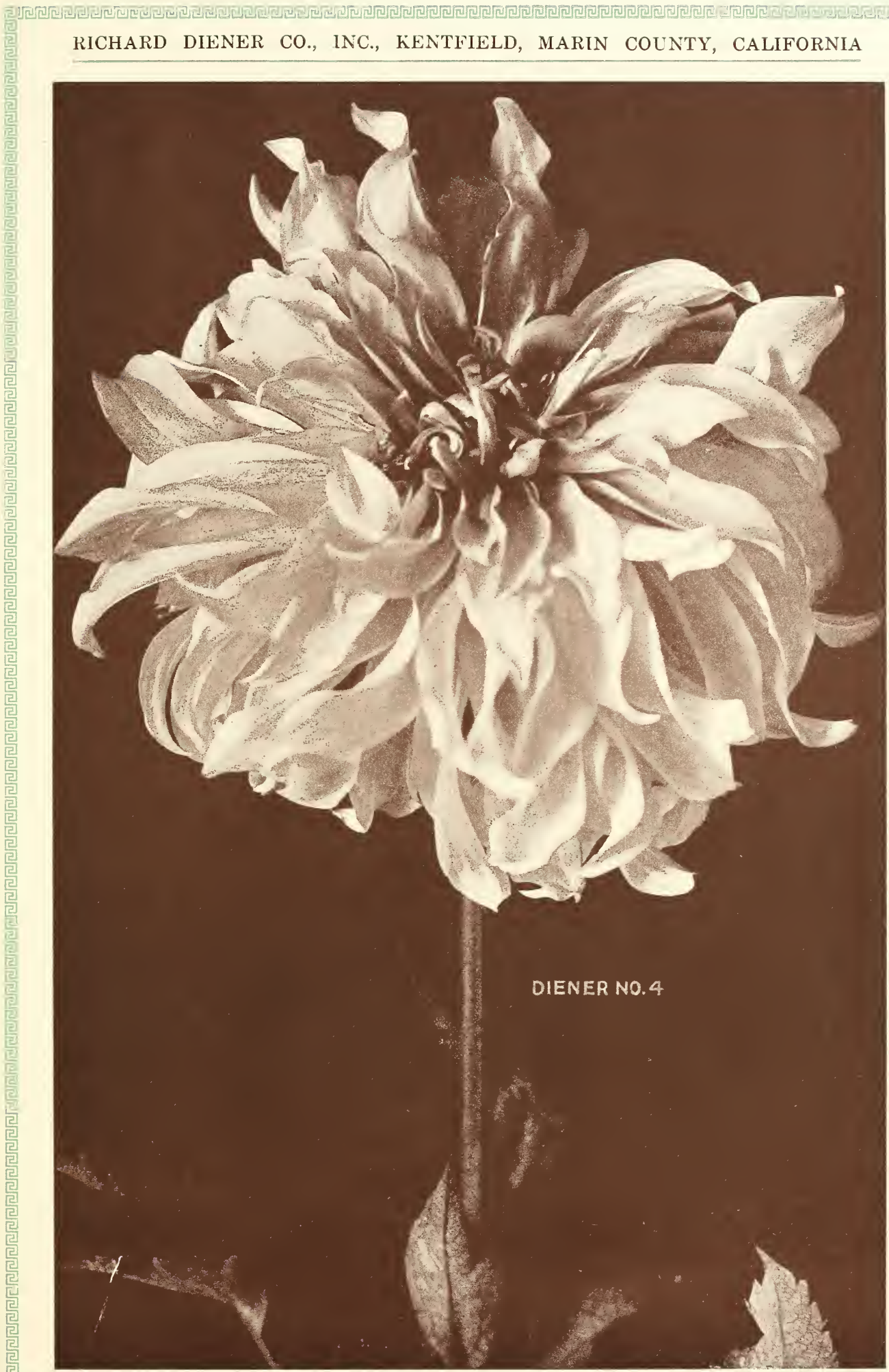

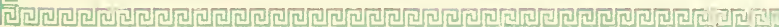


DIENER NO. 26.

A very much admired Decorative of pure white. No markings whatsoever. Wide open petals, blossoms large and full. Exceptionally fine stems and foliage. Good, clean grower.

DIENER NO. 27.

$\$ 5.00$ each

A semi-double of fine form. Liseran purple shading into pale olive-buff in the center. Large twisted petals, curling artistically. Very fine stems.

DIENER NO. 28.

$\$ 3.00$ each

Citron yellow heavily overlaid with pale Rose Purple. Very large decorative. Petals extremely wide and long. Wide open. Good, tough stems. A beauty.

DIENER NO. 29. $\$ 5.00$ each

An immense decorative of extremely heavy substance. Deep Nopal red. Outer rows of petals tipped with old gold. Very iridescent and velvety. Good, long, wiry stems.

DIENER NO. 30.

$\$ 3.00$ each

Liseran purple with rose purple underground. Long, broad petals, slightly curled and twisted. Immense flower. Stems long, very heary and wiry, and of erect build.

DIENER NO. 31.

$$
\$ 5.00 \text { each }
$$

Enormous Decorative of Barium Yellow, overlaid with pinkish cinnamon and iridescent old rose. Very long, wide petals. Stems extra long and wiry and bear the blooms correctly.

DIENER NO. 34 .

$\$ 10.00$ each

A striking large Double. Very iridescent Rhodamine purple. Center Aster purple. Each pctal is unusually large and wide opcn. Long, wiry stems bear the blooms beautifully.

$$
\$ 5.00 \text { each } \quad \$ 50.00 \mathrm{doz} \text {. }
$$

\section{"HELIO"}

A very striking Pcony type of bright canary yellow. Stems long and wiry and hold the flowers correctly. Flowers of the very largest size and enormously free blooming. Strong and healthy grower. It is perfect in every respect-one of the finest productions on the markt.

$$
\$ 1.00 \text { each }
$$

$\$ 10.00 \mathrm{doz}$

\section{"APRICOT"}

Large semi-double. Jasper pink, overlaid with lemon chrome around the center. Shell nink underground. Very iridescent. The whole coloring blended together gives an apricot shading. fiorgeous flower. Stems long and strong. Petals are large and wide open, curling artistically. $\$ 1.00$ each

$\$ 10.00 \mathrm{doz}$

Peekskill, New York, September 11, 1923.

My Dear Mr. Diener:-Last Spring I purchased a package of your Mrs. Carl Salbach Dahlia sects, and I have succeeded in raising about fifty plants. During July I was in Chicago, and when I returned found that all of our hillside was pretty well dried up, as there had been only three or four short showers during the whole month. August was no better and we were forbiddel to use the hose by the village authorities. However, I had the dish and bath water saved, anc managed to keep the Dahlias growing, but of course their growth is much stunted under thesc adverse conditions. They are just now beginning to bloom. Seven or eight of the Salbach Seedlings have bloomed thus far and I am delighted with them. One is a beautiful clear pink and soft vellow peony perfectly formed. Another is a most unusual flower of old rose and tan, witl soft yellow peony perfectly formed. Another is a most unusual flower of old rose and tan, with
petals curled and twisted and all of the petals having three divisions. It looks more like a hugc Crego Giant Aster than like a Dahlia.

Then there arc two lovely white Peony Dahlias, one creamy, and the other a pure white, also a perfect white decorative. The first blossom being rather small. Then there is a large tango cluplex and a mauve pink duplex. They stand up as stiff and straight as a ramrod.

Being a Sister of Mercy with no money of my own at my disposal it has only been possiblc for me to buy a few of the cheaper priced Dahlias each year and I tried to add to my numbers for me to buy a en of red or magenta singles. Yours was the first expensive seed I have ever bought, and already will red or magenta singles. Yours was the first expensive seed I have ever

Hoping that you will forgive this letter from an entire stranger and that next year you will again give the public the opportunity of purchasing seed which your skill has produced.

Yours very sincerely, SISTER MARY IRENE, C. S. M.

Richard Dicner Co. Inc., Ientfield, Cal.

Savannah, Ga., Mar. 13, 1922

Gived (100) seed of your dahlias. I purchased a package of seed from you last spring (50 cent package) and I must say that I never saw such beautiful flowers as to size and shades of colors. Some of the flowers were of the cactus type and as large as $5 \%$ inches in dianeter.

$$
\text { Yours very truly, }
$$

E. J. RANKIN. 


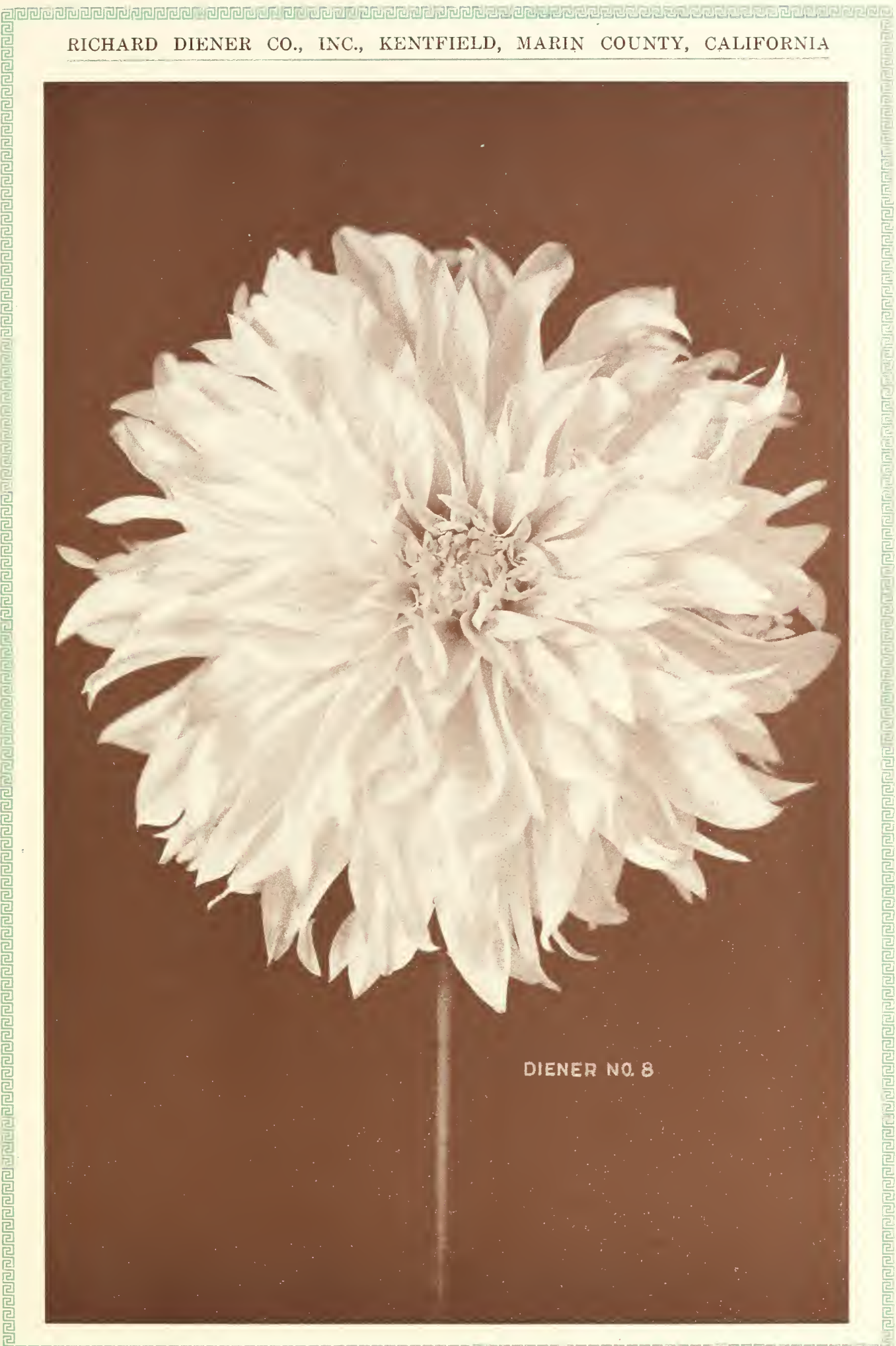

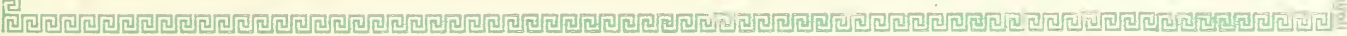




\section{Dahlia Seed}

Selected from the most up-to-date Dahlias in existence of all the most modern types. This mixture contains all colors. Our rainless California summers make it possible to get seed from the very double varieties, which is extremely difficult in states where it is rainy during the time when seeds should set. People handling our seeds this past year have written us of their successful results, which vou may see by the testimonials.

Price per package $(100$ seeds $) \ldots \ldots \ldots \ldots \ldots \ldots \ldots \$ .50$

\section{Dahlia Seeds from Mrs. Carl Salbach's Seedlings}

The new Dahlia-Mrs. Carl Salbach-is in our opinion the longest and strongest stemmed Dahlia we have ever seen. Most of the large Dahlias in existence now lack good long stems. For this reason. Mr. Diener made a great many crosses with the Mrs. Carl Salbach Dahlia, using the pollen of all the best varieties, and succeeded in raising several thousand seedlings which brought a percentage of $95 \%$ double and of coloring beyond description; also of very large size and the majority with very long stems. We saved all the seed of those seedlings, and so we are able to offer you something extraordinarily fine in Dahlia Seed.

Price per 100 seeds..................\$3.00

\section{Directions for Sowing Dahlia Seeds}

Dahlia seed should be spread out on a smooth surface of sandy leaf mold soil and gently pressed in with a flat board. Then shake some of the same soil over the seeds with a sieve, barely covering them. Keep them in a warm and moist place. Under no conditions let the soil dry out. Place glass or cardboard over the flats to conserve moisture. After the seeds begin to germinate, uncover the boxes and hang cheesecloth, so as to protect them from the blazing sun for a few days. Seeds will sprout unevenly. Some at once and others very much later.

After they are up 3 or 4 days, transplant them into two inch pots or into boxes three inches apart. Give the young seedlings plenty of sunshine, but keep them cool so as to have them sturdy when ready for the garden.

\section{Statice Sinuata}

(Everlasting) "Deep Blue"

A new, very valuable cut flower originated by us. It is a perennial which grows about 20 inches high and flowers continually. The flowers when cut will last for several years. This variety should be given a trial everywhere.

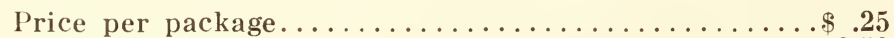

Price per pound.......................... 3.50

\section{Statice Sinuata Mixed Hybrids}

In colors of white, yellow, lavender, pink and lilac.

Price per package......................

\section{Directions for Sowing Statice Seed}

Statice seed should be sown in sandy leaf mold soil. Even off the ground well, then sprinkle the seed just as it comes over the surface and press them in firmly with a flat board. Do not cover. Water thoroughly and cover with a sack or burlap, which should be kept moist continually by sprinkling same 3 or 4 times a day. As soon as the young plants appear, which will be in 7 to 8 days, the sacks should be removed. Care should be taken to prevent the snails from attacking the young seedlings. Sprinkle lightly with air-slaked lime. 


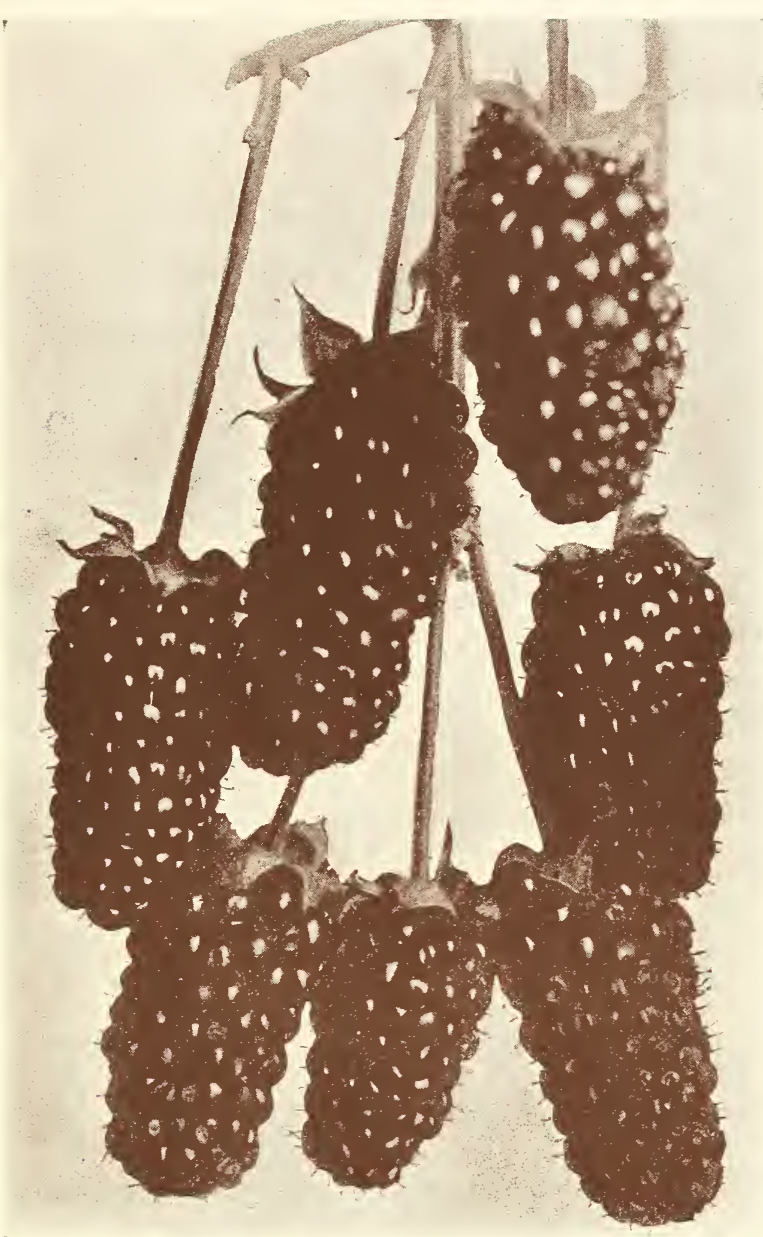

\section{The Cory Thornless Mammoth Blackberry}

(Actual Size)

After several years of trial the Cory Thornless Blackberry has proven to be the largest and best blackberry in existence. Acres and acres of this berry have been planted in California, Washington, and Oregon, and everywhere it has proven a success.

The vines of this blackberry are as free from thorns as a grape vine. A single berry reaches the size of a man's thumb. It has a flavor resembling the California Wild Blackberry. The core is very small and seeds are so fine that they are hardly noticeable when eating.

If you plant any berry at all, plant the Cory Thornless Blackberry, as it has never failed to bear.

This berry should be raised in bush form, not higher than five feet above the ground, or on trellises. In states where the weather goes below zero, vines must be covered with straw or sacks for the first two years (just like the roses), until the same become acclimated.

\section{PRICES}

Strong well rooted tips, carefully packed: Five tips for $\$ 1.00$ (smallest amount delivered); $\$ 2.00$ per dozen; $\$ 12.00$ per hundred; $\$ 110.00$ per thousand.

Transplanted plants: Three plants for $\$ 1.00$ (smallest amount delivered) ; $\$ 3.00$ per dozen; $\$ 15.00$ per hundred; $\$ 125.00$ per thousand.

Ready for shipment after January 1 st. 


\section{Vanderbilts' Hybrids}

These new Delphiniums produced by Newell F. Yanderbilt of San Rafael, California, are a (ross between the California Wild Delphinium (which are absolutely mildew free) and some selected Burbank's Hybrids. The result is an almost mildew free Delphinium. They are much larger than the best Delphiniums now existing, and are immensely valuable for the production of cut flowers, as they give three successive crops in one summer, and all summer long are full of fine, long spikes. They are suitable for cutting as wcll as for bedding. They are unquestionably the world's highest attainment in Delphinium, and anybody raising cut flowers will certainly find the world's highest attainment in Delphinium, and anybody raising cut flowers will certainly find them. They come in all shades from light to dark blue. Almost all colors are iridescent. Try a package of the seed and you will never regret it, for they are sure moneymakers.

These Delphinium have won prizes wherever exhibited, in competition with other strains

Finst Prize, California State Floral Society Spring Show, 1914.

First Prize, P. P. I. E. Cut Flower Show.

Michel Medal, Floral Pageant, 1916.

First Medal, P. P. I. E. Garden Exhibit.

Trade Package ......................

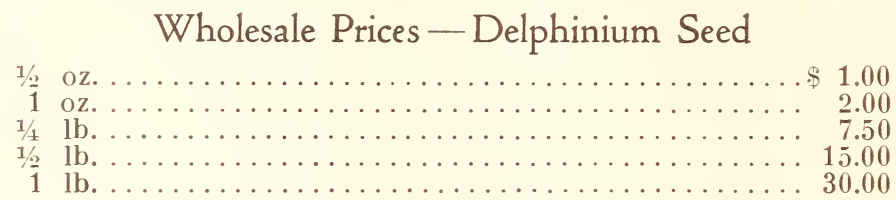

Delphinium seed can be sown out at any time of the year. Sow in boxcs or open cold frames. Do not cover more than the thickness of the seed. Give them little or no shade. Keep ground moistened until they sprout, which takes them from two to threc weeks. As soon as the plants are large enough, set out in the open. They will reach their full development in their second or third year.

\section{Vanderbilt's Hybrid Delphinium Plants}

Price per dozen plants..................\$2.50

Price per 100 plants..................... 18.00

Price per 100 plants $(2$ yrs. old) $\ldots \ldots \ldots \ldots \ldots \ldots \ldots .25 .00$

In the states where the ground freezes we recommend not to have the plants sent until ready to plant, as they should be set out immediately after they are received. We advise you not to have them sent in zero weather.

\section{Primula Malacoides Diener Hybrids}

They are the results of three successive crosses between Primula Malacoides and Primula Obconica. The flowers are 2 to 3 times larger than Primula Malacoides and the colors are much deeper. They range from a deep lavender to almost red. The plants grow stronger and are not so apt to rot off easily. They are a great improvement on Primula Malacoides. These flowers are favorites for pot plants and winter cut flowers.

Per package (about 300 seeds) ................ .50

Per dozen packages.......................... 5.00

\section{Diener's Hybrid Freesia Seed}

The size of the flowers are 2 to 3 times larger than Freesia Purity, from Snow white, Lilac, Lavender to deep blue in all shadings of the rainbow. Having strong stems, they are the highest perfection of the Freesias today. We offer a limited amount of seeds this year at:

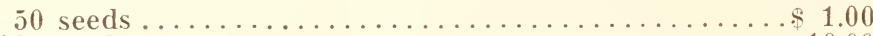

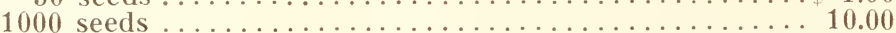

Seeds should be sown in sandy leaf mold in boxes, separating them one inch apart, one-eighth of an inch deep in the ground. When proper care is given them, the seedlings will all flower four months from date of sowing, but size of flowers will come out the second year. 


\section{The Aztec Bean}

It is one of the largest beans known. It grows in bush form, just like the Lima Bean, and is a perennial in places where it is not too cold or too frosty. It grows like any other bean, but stands a great deal of drought. Its flavor is unexcelled. Dry beans can be cooked just like Lima Beans. It is very prolific, and we recommend anyone who raises beans to give it a trial.

Price per package....................\$.50

In the state of Virginia, last spring, all the early vegetables were killed by a very heavy late frost. The frost, however, did not hurt the Aztec Bean in the least.

\section{Cucumber Seed "Sensation"}

This cucumber has proven to be the most valuable variety for forcing under glass and in hotbeds as well as for outdoors. In a greenhouse with a temperature of 70 degrees, the fruit will be ready for market one week after flowering. The fruit reaches the length of 12 inches and is of a deep green color. One plant will bring from 270 to 300 perfect fruit in one season. In order to get more fruit from a vine, never allow any of the fruit to ripen on the plant; as soon as they reach their full size, pick immediately.

Price per package.................... .50

\section{Gladiolus Culture}

\section{SOIL}

Gladioli will grow in almost any kind of soil, but a heavy clay or adobe soil containing some sand seems to be the ideal ground for them. The ground should be well spaded in the spring, and if it is not rich enough, some bone meal, or old, well-rotted fertilizer should be spaded under. Do not use any fresh straw fertilizer, as this always has a bad effect on the health of the bulb.

\section{PLANTING}

As to the depth that the bulbs should be planted, that is a question of locality. Where there is lots of rain and storms the bulbs should be planted as deep as three inches, but we find that the bulbs do best, taking into consideration growth and increase of bulblets, when they are planted to a depth of about one inch or thereabouts.

Early planting gives the largest amount of bulblets, and later planting the least. By planting at intervals of 10 days, from the early part of April to the first of July, a succession of blooms can be had until late Fall. If bulbs are planted in smaller quantity, plant the smaller ones first, as this gives them a better chance to develop, and, as the larger ones are of greater substance, they are better fitted to be kept longer. If planting on a larger scale, plant at intervals using both large and small bulbs, thereby spreading the blooming season still further.

Gladioli, in order to develop to perfection, should be planted in an open spot where the sun will strike the whole day long. Where they are planted in the shade or under trees, their growth is stunted and the colors do not come out to perfection. As our Gladioli varieties are naturally very large, we do not recommend any heavy fertilizing. Heavy fertilizing will produce much larger flowers, but at the same time will lessen the vitality of the bulbs. In such cases the bulbs will easily die during the winter as a result of it. We recommend top fertilizing with dry blood and bone meal (equal parts of each), mixed with the soil, working it in with a hoe. Liquid Cow or Sheep manure (if not overdone) is very good too, without any harm to the bulbs.

\section{CULTIVATING}

At all times from planting until the bulbs are taken up, the ground should be well cultivated and free from weeds. This is very essential. Some provisions must be made to protect the stalks from being broken down by the wind or rain, as when the buds begin to form, the spikes get very heavy and fall easily. The best wav to do this is to plant the Gladioli in rows; then drive in laths, one in each side of the row, every four feet apart; then run two lines tied to the laths on each side, one line two feet high and the other three feet high. This is a quick and easy way. 


\section{WATERING}

Newly planted bulbs should have no water under any condition, as the moisture of the ground is enough to bring them out. Do not water until they are from 6 to 8 inches high. We have found from experience that much danıage can be done to the bulbs in this way. After they reach the height of 6 to 8 inches they want all the water they can get. Give them plenty until they begin to flower. Even after they have flowered they should have just as heavy a watering for at least a month. When the flowers are open do not water while the sun is shining, as this will surely ruin the blooms. Water either early in the morning or late in the evening.

\section{CUTTING}

When the flowers are cut there should be at least 4 full leaves left on the bulb, and the cutting of the flowers should be done by punching the point of a knife sideways into the stem, and then by cracking the stem over. Many people make a mistake when cutting flowers, as they cut all of the leaves away. By doing this the shock on the bulb is so great, that it may die over the winter. The new bulbs build out after flowering and need as many leaves as possible to accomplish this. For this reason-spare the leaves. Many people do not allow seeds to form, but this is wrong. Seed pods should be allowed to form as this helps build out the bulb and is of real benefit to it.

\section{DIGGING AND CURING}

When digging the bulbs, do not cut the leaves off at once. Leave them on at least for 10 days or two weeks. Pile them out in the open so that the green leaves will throw the moisture out of the bulbs and help the bulbs to ripen naturally. After the green is cut off, leave them in a frost protected place for at least another two weeks. Then test the bulbs to see if they come off the old bulb readily. There is a certain period, determined by the climatic conditions, when the bulbs can be removed easily. Then the bulblets should be taken off and stored for next year's seeding. The outer husks should be taken off the bulbs, leaving them with a clean and silky appearance. The rest of the covering should be left on, as this is for the protection of the bulbs.

Great care should be taken that this operation takes place at the proper time, as on the other hand if they are left in the outer shell too long, they will become hardened again.

\section{STORING}

They should be put into well ventilated boxes not more than six inches deep. They should have a free circulation of air at all times. Keep dark if practicable.

\section{NOTE!}

Gladioli have their enemies, just as well as any other plants-insects and fungi. For this reason, in order to keep the plants healthy, one should take precautions against the aforesaid. A Bluestone Solution: 1 pound of Bluestone, 100 pounds of water. The bulbs should be dipped into the solution from 3 to 5 minutes just before planting. (Some use Formaldehyde Solution for the same purpose.) In ground where the leaves show too light a green, either treat it with Scotch Soot or sprinkle the ground with fresh Portland Cement. This will darken the leaves and make the plants much healthier. Under no conditions do we recommend nitrate of soda or any other chemical fertilizer.

In order to propagate Gladioli cquickly, we would suggest to cut large size bulbs in half. To do this correctly it should be done the day of planting; for if left out of the ground very long after being cut they may easily rot. By removing all the husk or silk, you will notice that the eyes are located on opposite sides, so divide the bulbs vertically through the center, so that each piece has at least one eye. The bulbs should be cut once only. No more than two halves from one bulb. We have been doing this with our bulbs, and this process has yet to fail us. In order to get the most bulblets from one bulb, they should be planted as early as possible. We find that the bulbs that have the top part of the bulb out of the ground have the most bulblets. 


\section{Petunia Culture}

Our new Ruffled Monster Petunias have brought this strain in very high favor all over the world. For this reason the cultivation of the same is being taken up everywhere on a much larger scale than ever before, and as many of our customers have asked for the best cultural directions for them, we are giving some instructions as to the best way of growing them in pots.

\section{TRANSPLANTING}

When the young plants reach the stage where they have from 4 to 5 leaves, they should be transplanted into $2 \frac{1 / 2}{2}$ to 3 inch pots, and after the roots are well filled out, which will be from 3 to 4 weeks after they have been potted, they should be shifted into 5,6 or 7 inch pots. When transplanting, great care should be taken that only the root part of the Petunia is in the ground, and all of the plant part is out of it. Plants, when transplanted, are often set deeper in the soil, and under such conditions, they either damp off, or it takes them a long time before they continue their natural growth. This is the most important point of all Petunia Culture, and should be taken very deeply into consideration; RATHER PLANT HIGHER THAN TOO LOW.

\section{SOIL}

Petunias are great feeders, and require rich ground. Any good compost or old hotbed soil, with about $1 / 6$ clean sand added is good. Bone meal and dry blood should be added in moderate quantities. This should be thoroughly mixed in the soil before the soil is used.

\section{LOCATION}

Petunias need plenty of light and hardly any shading. When raised in greenhouses or hotbeds, they should be as close to the glass as possible, and should also have plenty of ventilation. When planted in the open or in a bed, the sunniest and most open spot in the garden will be the best for them, but half shade will do if there is no other place available. For winter flowering in conservatories, they are the most satisfactory plant today, as they are continually in flower, providing the seed pods are removed, but if left on they will retard the blooming. Therefore, remove the seed pods as soon as they begin to appear.

\section{COMMERCIAL VALUE}

For florists, for Easter pot plants they are the most preferable of all flowers. Florists that have taken up this culture tell us their customers will buy our Giant Petunias first of all, when making their selections from a large display of choice flowers and plants. Commercially, they have an unlimited future.

Petunia plants out of three-inch pots, flowering, $\$ 2.50$ per dozen.

Prices for larger amounts on request.

From April to the end of June only.

We do not guarantee Petunia Plant Shipments to distances over 1,000 miles.

Richard Diener Co. Inc., Kentfield, Cal.

Scarsdale, N. Y.

Dear Sirs:-Last year I had Gladioli and Delphinium Seed from you. Both did so well. The glads are still to flower. The Delphinium are in bloom now and I liave never seen anything more beautiful. I cannot be without the Delphinium, so please send another packet at once, And oblige,

H. ADAIR.

Richard Diener Co. Inc., Kentfield, Cal.

Biggs, Calif., Oct. 17, 1922.

Gentlemen:-i am most enthusiastic over the Dahlias from your seed. One old rose cactus is over six inches in diameter, a wonderful flower, have a lilac decorative that is as large. There are very few really ordinary ones in the bunch.

The Gladioli bulbs all came true to colors in the catalog, and were much admired. My The Gladioli bulbs all came true to colors in the catalog, and were much admired. My
favorites were Mrs. Wm. Kent, which I thought glorious, and Anna Eberius. One bulb of Costa Rica favorites were Mrs. Wm. Kent, which I thought glorious, and Anna Eberius. One bulb of Costa Rica
and Capt. Baker threw out blooming stalks again in September, making two blooming periods for them.

Respectfully,

MRS. W. S. SCHILL.

Richard Diener Co. Inc.,

Columbus, Ohio, July 25, 1923.

Gentlemen:- iVas very much pleased with all of the bulbs that I ordered this season. They are all healthy and are giving good returns in beautiful flowers. You surely are the best Gladiolus dealer. Thanking you kindly, I am,

Yours truly,

FLOYD ATWILL, JR.

Richard Diener Co. Inc.,

Washington, D. C., July 26, 1923.

Dear Sirs:- Please send me your catalog of seeds and bulbs. I am very much pleased with the Petunias I have, from some of your seeds, that I got from a friend here. I have had some that measure 5 $\frac{1}{4}$ inches. I have not seen Petunias anywhere that could in any way compare with them.

Yours truly,

L. C. GREENE. 


\section{Some Testimonials}

In order to save space, we have taken only those parts of letters that relate to our productions.

Mr. Richard Diener, Kentfield, Cal.

Detroit, Michigan, August 28th, 1923.

Dear Sir:- "D. J. Whitney" with its 20 blooms and 8 fully opened is a remarkable flower of lively hues and in a garden full of Glads can hardly be passed unnoticed, yet.

"Mrs. Leon Douglas" with the same general eolors is larger as well as deeper in her markings. Her large spike is well furnished with a double row of blooms, widely expanded, like those of an exeeptional amaryllis. One spike of this variety to a vase is all it needs and all it holds. It will be a hard job to beat this lady.

"Pola Negri"- 16 bloons in two rows are borne on a strong and long lasting spike. Most of them are wide open at onee. The long eurved petals are arranged very symmetrically, whieh adds to their splendor, and artistie toueh, new in the gladioli. You eannot help giving Pola another look.

"Anna Eberius" enjoys too well an established popularity to need new eomments.

"Henry Ford" is yet of a more brilliant lustre, bigger bloom and a stronger stem. The blooms open like those on a good size Diener's Ameriean Beauty. Now double that and you will have the right size of this beautiful bouquet of only one spike, eonsisting of 20 blooms wide open at onee. However, you look at Henry and he is full of pep and vim.

"Caroline Esberg," 15 blooms, 8 open at onee. A real red one that lasts and is afraid neither of seorching sun nor stormy breezes.

"Katharine F. Hess," 20 blooms, 8 open at once. Big, clear, bright and impressive. It does not take her long to make friends.

"Nrs. Riehard Lohrman" being tied on to a post, had no ehanee to kink. 22 blooms, 11 open. She is straight, strong and wiry. The harmony of pleasing soft eolor in both buds and blooms is beyond critieism. Mrs. Lohrman is surely a magnifieent flower.

"Norma Krausgrill," 21 blooms, 9 open. She is something like Jaek London, yet a little deeper and elearer and softly ruffled. No danger of her being left alone.

"Mrs. Truxton Beale," 16 blooms, 5 open, a flower of neither mueh eolor or many blooms, but what is open, is of "substanee that eounts.

"Elf" and "Marin" are two good white flowers. The beautiful yellow buds in one as well as the greenish hues in the buds of the other, detraet nothing from these long lasting white varieties.

"Mrs. Lillian Guernsey" is a beautiful lily-like flower. It is a yellow glad that is sure to win you as well as all your friends.

The three odd ones worthy of praise are "Rose Ash," "Mr. Henry Beehman" and "London Smoke." The petals of the first one are shy neither in attractive eurling, nor beautiful waving or soft rumling, the seeond possesses a touch of iridescenee hard to describe and the third one with its large blooms is of a nore definite eolor. They all are robust and long lasting. In their presince niany varicties of name pale like eleetrie light at noon timc. Very respeetfully,

ADAM STEINHAUER, M. S.

Corry, Pa., April 14, 1922.

Riehard Diener Co. Ine., Kentfield, Cal.

Dear Sir:-The shipment of Gladioli arrived, and I surely appreciate the quality of bulbs sent and your liberality in "Gratis" bulbs. I ordered a mueh larger amount of Gladiolus from a specialist in this flower alone who is known throughout the states, and the shipment was far below your quality. I surely thank you.

Yours very truly,

Y. D. DESMOND.

Mr. Richard Dicner, Kentfield, Cal.

Boston, Mass., May 20, 1922.

Dear Sir:-The blackberry shrul)s arrived in splendid eondition, being still moist and fresh. Are now doing well in garden.

Richard Diener Co. Ine., Iientfield, Cal. Very truly yours,

MISS G. EVELYN GREENE.

Dear Sirs:-The Gladioli received from you were something grand, espeeially Mrs. Mary Stearns Burke, Jonathan Webb and Frank J. Symmes. Never saw sueh large bulbs. F. J. Symmes, at digging time last fall, measured 3 and $3 / 4$ inches aeross, and stood $6 \mathrm{ft}$. 2 in. high. I also had some of your Diener Tomatoes. Proeured plants from our local florist, and had some immense fruit, I think the finest flavored tomatoes I ever ate. Yours sineerely,

WM. W. BROOKS.

Riehard Diener Co. Ine., Kentfield, Cal.

Dundas, Ont., Canada, Mar. 8, 1923.

Dear Sirs:- I have bought quite a few of your Gladioli from loeal growers and have had wonderful sueeess with them. It pays to get the best for good results. Am sending for your Petunia seed today. Mr. Kennedy of Stratford reeommended you very highly to me. He said he had them measure 7 to 8 inehes across. Isn't that wonderful? He said that if I wanted to show the people of Dundas something wonderful in Petunias botl in size and eolors to send to Diener. Hoping to hear from you soon,

I am,

MRS. JACK IVILSON.

Richard Diener, Kentfield, Cal.

Los Banos, Cal., Jan. 4, 1922.

Dear Sir:-I must say that the Diener Tomato is the finest flavored Tomato that I ever ate. They started bearing in September and we had them all winter. When there was danger of frost, I picked all the large green ones and put them in a dark eupboard and we had the finest kind of 1 picked all the large green
ripe tomatoes all winter.

Oblige,

MRS. MITTIE DILSE. 


\section{List of Named Gladioli Originated by Richard Diener in Alphabetical Order}

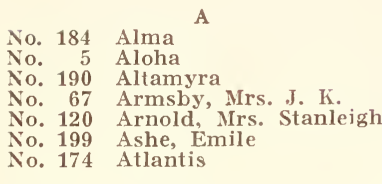

\section{B}

No. 164 Baker, Capt. Asher Carter

No. 180 Beale, Mrs. Truxton

No. 335 Bechman, Mr. Henry

No. 234 Belvedere

No. 336 Bennett, Dr. F. E.

No. 244 Bolinas

No. 209 Bothin, Genevieve

No. 191 Bothin, Mrs. H. E.

No. 328 Bubbles

No. 90 Burke, Caroline Frear

No. 188 Burke, Mrs. Mary Stearns

\section{C}

No. 257 California Giant

No. 305 Carbone, J. A

No. 361 Carmelita

No. 203 Casement, Sir Roger

No. 367 Cattlaya

No. 70 Coffin, Lillian Harris

No. 102 Collins, Ina

No. 187 Costa Rica

No. 39 Cothran, Dr. Lincoln

No. 145 Cothran, Mrs.

No. 82 Coulter, John Merle

No. 297 Cunningham, Mrs. Robert

$$
\text { D }
$$

No. 281 Del Monte

No. 96 Diener, Richard

No. 23 Diener's American Beauty

No. 166 Diener's White

No. 175 Douglas, Mrs. Leon

No. 10 Eberius, Anna

No. 237 Edison, Thos. A.

No. 220 Eldiva

No. 321 Elf

No. 101 Else Rose

No. 266 Esberg, Caroline

No. 334 Eudora

\section{$\mathrm{F}$}

No. 32 Fairfax

No. 338 Farrar,Geraldine

No. 176 Fay, Miss Maud

No. 216 Fernekes, Martha

No. 177 Field, Joseph

No. 232 Flame

No. 339 Ford, Henry

No. 241 Ferdericks, Victoria

\section{G}

No. 269 Geisha

No. 189 Gerberding, Elizabeth

No. 213 Guernsey, Mrs. Lillian

No.

\section{$\mathrm{H}$}

No. 315 Hardee, Elizabeth

No. 173 Hawaii

No. 107 Heart of Juanita

No. 318 Hess, Katharine $F$.

No. 98 Hubbard, Mable

No. 200 Hyde, Mr. H. A.

$$
\text { I }
$$

No. 295 Ibanez, Blasco

$$
\mathrm{J}
$$

No. 205 Jordan, David Starr
$\mathrm{K}$

No. 136 Kent, Adeline

No. 1 Kent, Mrs. Willian

No. 130 Kent, Thomas T.

No. 206 Kent, William

No. 89 Krausgrill, Norma

No. 355 Lagunita

No. 4 Lillie, Sarah

No. 312 Lohrman, Mrs. Richard

No. 38 London, Jack

No. 221 London' Smok

No. 330 Loreley

No. 317 Lucia

No. 236 Marin

No. 324 McGaviu, William

No. 19 McNally, Myrtle

No. 277 Michelena, Beatriz

No. 329 Moonbeam

No. 31 Moore, President C. C.

No. 283 N

No. 293 Negri, Pola

No. 358 Nolene

No. 320 Olson, Esther

No. 268 Papilio

No. 226 Pasadena

No. 282 Phini

No. 331 Phipps, Mr. W. H

No. 326 Phylos

No. 311 Plath, Gladis

No. 50 Queen of the Night

No. 162 Rinehart, Mary Roberts

$\begin{array}{ll}\text { No. } & 162 \\ \text { No. } & 83 \text { Richardson, Mrs. Willard }\end{array}$

$\begin{aligned} \text { No. } 83 & \text { Richardson, } \\ \text { No. } 332 & \text { Rose Ash }\end{aligned}$

No. 327 Rosenthal, Dr. Adolplı

No. 198 Ross Valley

\section{$\mathrm{S}$}

No. 240 Salome Jane

No. 22 San Anselmo

No. 304 San Jose

No. 9 Sausalito

No. 276 Shaw, Bernard

No. 117 Sierra

No. 165 Skiff, Dr. Frederick J. V.

No. 298 Sonoma

No. 285 Spreckels, Mrs. Rudolph

No. 246 Stahlbaum, Mimi

No. 13 Sunset

No. 204 Symmes, Frank J. $\mathbf{T}$

No. 286 Taylor, Ruth

No. 265 Thompson, Anuc

No. 196 Todd, Helen

No. 365 Torador

No. 354 Valdemar, Thais

No. 113 Vanderbilt, Mrs. Newell

No. 192 Victory

No. 337 Virginian

No. 341 Waikiki

No. 202 Walsh, Mrs, John R.

No. 193 Webb, Jonathan

Yo, 186 Vebb, Lillian

No. 24 Whitney, D. J.

No. 24 Whitney, D. J
No. 333 Wistaria

No. 218 Yosemite

No. 211 Zoe
Z 


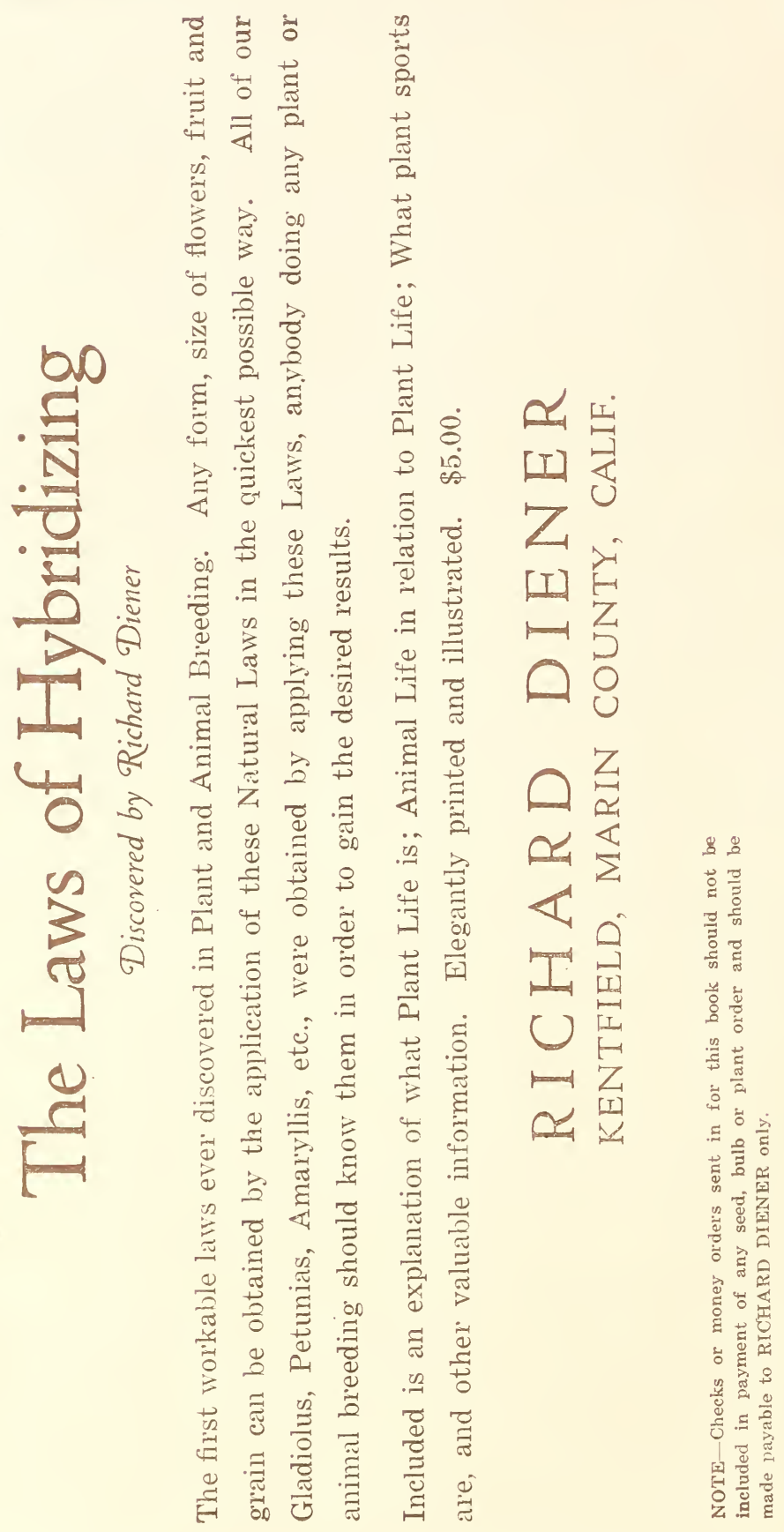


